\title{
Don't pay attention to high-calorie foods for a healthy weight: attention bias for food and the desire to eat
}

Citation for published version (APA):

Werthmann, J. (2014). Don't pay attention to high-calorie foods for a healthy weight: attention bias for food and the desire to eat. [Doctoral Thesis, Maastricht University]. Datawyse / Universitaire Pers Maastricht. https://doi.org/10.26481/dis.20140320jw

Document status and date:

Published: 01/01/2014

DOI:

10.26481/dis.20140320jw

Document Version:

Publisher's PDF, also known as Version of record

\section{Please check the document version of this publication:}

- A submitted manuscript is the version of the article upon submission and before peer-review. There can be important differences between the submitted version and the official published version of record.

People interested in the research are advised to contact the author for the final version of the publication, or visit the DOI to the publisher's website.

- The final author version and the galley proof are versions of the publication after peer review.

- The final published version features the final layout of the paper including the volume, issue and page numbers.

Link to publication

\footnotetext{
General rights rights.

- You may freely distribute the URL identifying the publication in the public portal. please follow below link for the End User Agreement:

www.umlib.nl/taverne-license

Take down policy

If you believe that this document breaches copyright please contact us at:

repository@maastrichtuniversity.nl

providing details and we will investigate your claim.
}

Copyright and moral rights for the publications made accessible in the public portal are retained by the authors and/or other copyright owners and it is a condition of accessing publications that users recognise and abide by the legal requirements associated with these

- Users may download and print one copy of any publication from the public portal for the purpose of private study or research.

- You may not further distribute the material or use it for any profit-making activity or commercial gain

If the publication is distributed under the terms of Article $25 \mathrm{fa}$ of the Dutch Copyright Act, indicated by the "Taverne" license above, 




\section{Don't pay attention to high-calorie foods for a healthy weight: Attention bias for food and the desire to eat}


(C) Copyright J Werthmann, Maastricht 2014

ISBN 9789461593146

Datawyse / Universitaire Pers Maastricht

Graphic design: Zwischengestalten

Art work: Alberto Ruggieri (Corbis) 


\section{Don't pay attention to high-calorie foods for a healthy weight: Attention bias for food and the desire to eat}

\section{Proefschrift}

Ter verkrijging van de graad van doctor aan de Universiteit Maastricht, op gezag van Rector Magnificus, Prof. dr. L.L.G. Soete, volgens het besluit van het College van Decanen, in het openbaar te verdedigen op donderdag 20 maart 2014 om 16.00 uur door

Jessica Werthmann

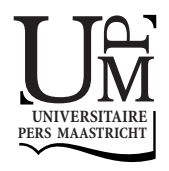




\section{Promotoren}

Prof. dr. A.T.M. Jansen

Dr. A. Roefs

Dr. C. Nederkoorn

\section{Beoordelingscommissie}

Prof. dr. A. Arntz (voorzitter)

Prof. dr. I. Franken (Erasmus Universiteit Rotterdam)

Prof. dr. P. de Jong (Rijksuniversiteit Groningen)

Prof. dr. M. Peters

Prof. dr. N. de Vries 


\section{CONTENTS}

Chapter 1 General Introduction

Chapter 2 Can(not) take my eyes off it: Attention bias for food in overweight participants

Chapter 3 The power of food: Attention bias for high-calorie food predicts weight gain in obese children

Chapter 4 Attention bias for food is independent of restraint in healthy weight individuals: An eye tracking study

Chapter 5 Desire lies in the eyes: Attention bias for chocolate is related to craving and self-endorsed eating permission

Chapter 6 Looking at food in sad mood: Do attentional biases lead emotional eaters into overeating after negative mood induction?

Chapter 7 Attention bias for chocolate increases chocolate consumption: An attention bias modification study

Chapter 8 General discussion

Summary

References

Acknowledgements 



\section{Chapter 1}

General Introduction 
Imagine a nice Saturday afternoon and you are on a shopping spree in the city centre. Wherever you go, you see delicious food: you notice a lot of great gourmet food stores, bakeries, ice-cream stands, and fast-food restaurants in the shopping streets and you do not seem to be able to take your eyes off the delicious food that is offered. Do you think that paying attention to these stimuli can influence your eating behavior? Some obesity researchers suggest that selectively attending food stimuli increases the risk of overeating palatable high-calorie food (Nijs \& Franken, 2012).

However, not everybody is equally susceptible to the impact of these food cues. Some people's attention may not be attracted to the omnipresent palatable food cues. Imagine a lean friend, who accompanies you on the shopping trip and ends up with buying a lot of new shoes, instead of food. An explanation for these differences in eating behavior could be that she did not even notice the sweet temptations in the surroundings, and instead her attention was focused on other personally relevant cues, such as shoes. Research indeed suggests that healthy weight individuals do not have biased attention for food cues compared to obese individuals, at least when they are satiated (Castellanos et al., 2009).

Possibly, another friend, who is on one of her diets again, might also have noticed the nice food offers, but then tries to ignore the food and follows the lean friend to the shoe shop. Some studies found evidence that individuals who try to refrain from eating tempting but high-calorie food are distracted by food cues (Hollitt, Kemps, Tiggemann, Smeets, \& Mills, 2010; Papies, Stroebe, \& Aarts, 2008). These examples illustrate how an attention bias for food can differ, depending on individual weight and motivational characteristics and how the interplay of biased attention for food and individual characteristics can influence eating behavior. Both, craving for food (i.e., appetitive motivation) and worrying about food (i.e., eating restraint) can distort attention for food, although the exact attention process that is affected by these motivations or emotions has not been specified.

The central idea of the present thesis is that an attention bias for food contributes to overeating, difficulties to lose weight, and obesity. One aim of this thesis was to systematically investigate whether an attention bias for food cues is distinctly related to BMI and obesity-associated eating behaviors, such as restrained or emotional eating. Another aim of this thesis was to test if and how attention biases for food are (causally) related to craving for food and food intake. After introducing the topic of an attention bias in a "toxic" food environment, theoretical and methodological considerations are discussed concerning an attention bias for food. A brief review of existing empirical evidence regarding attentional biases for food and obesity follows. Subsequently a short outline of this thesis with a brief description of the conducted studies is presented. 


\section{Surrounded by a "toxic" food environment}

The prevalence of obesity is rising worldwide. Obesity is defined by a body mass index $\left(\mathrm{BMI} ; \mathrm{kg} / \mathrm{m}^{2}\right)>30$ and overweight by a BMI $>25$. According to the World Health Organization (WHO) more than 1.4 billion adults worldwide were overweight and more than half a billion obese in 2008 (World Health Organization, 2013a). Obesity is not only increasing dramatically among adults, but also among children. For example, in Europe $12.5 \%$ of all children under five years are overweight (World Health Organization, 2013b). In the Netherlands, 36.9\% of women were overweight or obese, $46.1 \%$ of men were overweight or obese and $12.4 \%$ of children under 12 years were overweight or obese in 2012 (Centraal Bureau voor de Statistiek, 2013). Obesity and overweight constitute a serious risk for psychological and physical well-being. Obesity is associated with internalizing mental disorders, such as depression, and a higher risk for diabetes, heart diseases and mortality in children and adults (Cali \& Caprio, 2008; Harriger \& Thompson, 2012; Hu, 2003; Kalarchian \& Marcus, 2012; Mokdad, Marks, Stroup, \& Gerberding, 2004; Pulgaron, 2013; World Health Organization, 1998). The WHO estimates that 2.8 million people die each year due to the adverse consequences of being overweight or obese (World Health Organization, 2013a).

Ultimately, obesity is caused by a long-lasting imbalance of energy intake and energy expenditure. This imbalance is, at least in our current society, mainly caused by excessive food intake (Hill, Catenacci, \& Wyatt, 2005; Van Zant, 1992). It is generally agreed upon that our current food environment is in this respect "toxic" (Hill \& Peters, 1998; Wadden, Brownell, \& Foster, 2002). Our food environment is characterized by an abundance of palatable, high-calorie, energy-dense, cheap and convenient food that is constantly available and promoted aggressively. Living in such an environment poses a serious challenge for weight maintenance. Yet, not everybody is equally susceptible to the temptations of this obesogenic food environment. The way in which someone perceives and reacts on this challenge, thus cognitive processes, could be responsible for these differences in susceptibility. Thus, it is important to study psychological mechanisms that contribute to successful healthy weight control and/or that facilitate weight gain. An attention bias for food in an obesogenic environment could be an important cognitive mechanism related to overeating and knowledge on attentional biases could be used to design effective weight-loss interventions.

Our environment contains more stimuli than we are able to process. Attention is a crucial factor for the selection of relevant information for further processing and in that respect determines which aspects of our environment are brought to our awareness. In general, two attentional mechanisms can be distinguished: (1) Attention prioritizes relevant information and this selection mechanism can be involuntary (i.e., a bottom-up process, for example when we see an ambulance), or 
(2) Attention can be steered voluntarily (i.e., a top-down process, for example when we search for something). A food-related attention bias refers to selective attentional processing of food cues, including both voluntary and involuntary processes. Early attentional processing is associated with a more involuntary, less controlled mechanism, whereas later attentional components are thought to reflect the slower top-down mechanisms of voluntary or more controlled attentional processing (e.g., Knudsen, 2007; LaBerge, 2002). It has been argued that selectively attending food cues might have been an adaptive advantage during evolution of mankind because being able to spot food quickly might have contributed to survival (Castellanos, et al., 2009; Nijs \& Franken, 2012). However, in an environment where food is abundant and food cues are designed to attract attention and are marketed aggressively, food-related attentional allocation could increase the chance of overeating and ultimately weight gain. Thus, in an obesogenic food environment having an attentional bias towards food cues could be critical for overeating.

\section{Attention is biased by craving and by concern: two sides of the same coin?}

Attentional biases for food in the context of obesity could constitute a problematic (cognitive) factor contributing to the failure to adhere to a healthy diet (e.g., Papies, et al., 2008). In line with this suggestion, current research showed that a previously learned rewarding experience might steer and attract attention, even when new goals have been formed (Anderson, Laurent, \& Yantis, 2013; Hickey \& Theeuwes, 2011). Applied to food-related biases in the context of obesity this might mean that once an attention bias for food is present, it might be difficult to ignore high-calorie food cues even during a period of actual or intended dieting. Moreover, theoretically, attention biases can reflect appetitive as well as aversive motivations (Frewen, Dozois, Joanisse, \& Neufeld, 2008). For example, according to addiction research, attention biases reflect approach motivation (thus drug-related cues are preferentially processed), whereas anxiety research suggests that attention biases are caused by worry (for example, focusing on negative or threatening cues). When it comes to attention for food, attention biases could reflect both: eating motivation (thus appetitive approach) as well as eating restraint (thus worry about food and weight). In the following, both theoretical accounts are summarized briefly.

Commonly, attention for food in the context of obesity is viewed in line with theoretical considerations and research evidence from the field of addiction research. According to addiction theories, an attention bias for desired (substancerelated) cues is a major force in drug seeking and drug taking behaviour. For example, according to the incentive sensitization model by Robinson and Berridge 
(Berridge, 1996, 2009), addictive cues signalling the imminent drug consumption gain incentive properties during a conditioning process of repeated signalling and subsequent drug consumption. As a result of this process, these stimuli become salient in the environment and are then potent to "grab attention" of drug users. This model has been extended and has been adapted as an explanation for overeating in the context of obesity (Nijs \& Franken, 2012). In this respect, a similar mechanism has been proposed: through a conditioning process based on the rewarding effects of food intake, cues (such as the sight of food) can gain incentive salience and thus become potent to attract attention. It has further been posited that attention for food cues and craving stand in a reciprocal relationship: attention for food cues is thought to evoke food cravings, whereas also the opposite is possible: craving for food steers attention for food cues (e.g., Nijs, Muris, et al., 2010; Smeets, Roefs \& Jansen, 2009). Thus, according to this addiction-account of overeating, obesity should be associated with approach attentional biases reflecting eating (enjoyment) motivation.

Yet, according to an anxiety-based account of eating disorders, researchers have proposed that an attentional bias for food could be related to fear of gaining weight or losing control over eating (Giel et al., 2011; Lee \& Shafran, 2004). A number of studies in the field of eating disorders on attentional processing have indeed relatively consistently shown that individuals suffering from anorexia nervosa, bulimia or binge eating disorder express an attention bias for food cues (see for reviews, e.g., Brooks, Prince, Stahl, Campbell, \& Treasure, 2011; Dobson \& Dozois, 2004; Faunce, 2002; Lee \& Shafran, 2004). Whereas most of these studies did not address the direction of attention when processing food cues (attentional approach of food or attentional avoidance), it has been suggested that food-related worry might be related to increased vigilance (thus early attentional approach) and/or later avoidance of further processing (e.g., Giel, et al., 2011). Thus, according to theory and dovetailed by empirical findings in eating disorder patients, it is also possible that eating-related worries, or dieting intentions, are associated with an attention bias of food cues.

In sum, whereas some models postulate that an attention bias for food reflects appetitive motivation in obese individuals, other models of food-related attention bias suggests that an attention bias for food reflects anxiety for food or food/calorie concerns. This implies that the interpretation of an attentional bias for food in obese individuals may not be so straightforward as sometimes is suggested. Considering that an obese person undertakes many dieting attempts (Andreyeva, Long, Henderson, \& Grode, 2010; Jeffery et al., 1984; Williamson, Serdula, Anda, Levy, \& Byers, 1992), it is not immediately clear whether an observed attention bias reflects a craving for calories or calorie concerns - or both. It seems important to take into account one's restraint-status and whether a person is currently dieting, besides the weight status when measuring attention bias for 
food in the context of obesity. Moreover, an accurate paradigm is needed, which can distinguish between attentional approach and avoidance, and between early and late components of attention bias. This is necessary to more fully understand the role of an attention bias for food in the aetiology and maintenance of obesity.

\section{Assessment of attention for food: many roads lead to Rome?}

There is a large variety in conceptualizations of attention bias, attentional components or underlying attentional mechanisms and the respective attention paradigm, which makes it difficult to summarize the current state of the art in the field of food-related attention biases in the context of obesity. At least four different methodologies have been applied in previous studies to asses attentional biases for food: (1) measuring response latencies or the calculation of an interference effect during a food Stroop task, (2) assessment of response latencies during a visual probe task or visual search task (3) a combination of the visual probe task or a visual search task with the recording of eye-movements, (4) assessment of ERPs during a free-viewing session of food images. In the following, these methods will be discussed briefly (see Table 1).

During the food-Stroop task (Williams, Mathews, \& MacLeod, 1996), coloured food and neutral words are presented, and participants are required to indicate the colour of the word as quickly as possible, irrespective of the meaning of the word. The Stroop interference score is calculated by obtaining a difference score between the average response latency on food versus neutral trials. This interference score is thought to reflect biased attention. That is, if the response latency is relatively prolonged on food trials, it is concluded that there is an attentional bias for food stimuli. A disadvantage of this paradigm is that it does not inform on the underlying attentional processes. The slow-down in colour-naming could be caused both by increased attention for the semantic meaning or by avoidance of processing the stimulus word altogether (Field \& Cox, 2008). Moreover, it is unclear which attentional components are reflected in the interference effect: While it has been argued that the Stroop effect could reflect a relatively early attentional process (i.e., involuntary semantic processing) (Cox, Fadardi, \& Pothos, 2006), results of a meta-analysis suggested that it is more likely to reflect later attentional processes (Phaf \& Kan, 2007). Taken together, a disadvantage of the food-Stroop task is that it is not clear which attentional component (early or later attentional processes) is captured, and that the interference effect cannot provide information on direction (approach versus avoidance) of the attentional process.

The visual probe task (MacLeod, Mathews, \& Tata, 1986) is a standard attention paradigm that relies on the assessment of response latencies. During this task, two stimuli (a critical stimulus and a neutral stimulus) are presented side by side 
on the computer screen for a fixed duration (typically $2000 \mathrm{~ms}$ ). Then, both stimuli disappear, and a small probe appears in the position of one of the stimuli. Participants are instructed to press a corresponding key on the keyboard to indicate the location of the probe (e.g., left or right). The logic of this task presumes that participants react faster to indicate the position of the probe if their attention was already directed to the location (thus on the stimulus) in which the probe appears. An advantage of the visual probe is that it is possible to distinguish early and later attentional processes, by including different presentation times: a stimulus duration of $100-500 \mathrm{~ms}$ is thought to assess initial orientation, whereas longer stimuli presentations (500 $\mathrm{ms}$ and above) are thought to assess maintained attention (e.g., Mogg, Bradley, De Bono, \& Painter, 1997; Mogg, Bradley, Miles, \& Dixon, 2004). Moreover, the calculation of a response latency based attention bias allows for the interpretation of the direction of attention: the mean response latency in congruent trials (when the probe replaces the relevant picture) is subtracted from the mean response latency in incongruent trials (when the probe replaces the neutral stimulus). According to this calculation positive bias scores reflect attentional approach and negative bias scores suggest attentional avoidance (Mogg, et al., 2004).

Yet, the indirect assessment of attention allocation by measuring response latencies remains imprecise: Response latency differences may stem from sensory inhibition or response competition effects and thus do not directly inform on the underlying (visuo-spatial) attentional mechanism that is involved (e.g., Field \& Christiansen, 2012; Field, Munafo, \& Franken, 2009).

A more direct assessment of attentional processes can be obtained by eye tracking or the assessment of event-related potentials (ERPs) for food cues during electroencephalographic (EEG) activity (e.g., Field, et al., 2009; Nijs, Franken, \& Muris, 2008). An advantage of these assessment methods is that attention allocation is measured more directly and therefore different attentional processes (such as early and later attention allocation) can be easily (and dynamically) disentangled. Moreover this direct assessment can inform more accurately on the direction of the attention effect (thus whether attentional approach or avoidance is present). The study of attentional components is important because research on attentional biases in substance abuse disorders showed that early versus later components of an attentional bias were differentially related to substance abuse (Field, Mogg, Zetteler, \& Bradley, 2004). For example, heavy drinkers had a strong attentional bias in maintained but not in early attention (Field, et al., 2004). As already mentioned before, it is crucial to determine the direction of attentional processes because theoretical accounts suggest that an attention bias could either capture appetitive motivation or eating restraint and it has not been clarified if eating restraint (thus worry about food and calorie intake) results in attentional approach or in attentional avoidance of tempting food cues. 
To conclude, currently, a direct measure of visual attention, such as eye-tracking, is most informative on the time course and the direction of attention processes. Eyetracking provides a direct index for overt visual attention, thereby generating a dynamic and ecologically valid measure of visual attention processes (Bradley, Mogg, Wright, \& Field, 2003; Field, Mogg, \& Bradley, 2006; Field, et al., 2009). Another advantage of eye movement recordings is that it is easy to accurately disentangle distinctive components of attention allocation processes, such as initial orientation and attention maintenance. Moreover, due to the direct recording, a precise calculation of the direction of attention (looking towards or away from relevant stimuli) is possible. Thus, this measure might be most capable to provide detailed information on attention processing of food cues.

\section{Attention bias for food and obesity: empirical findings are not eye-openers}

Empirical evidence for food-related attentional bias and overeating or obesity is mixed. In short: Some research suggest that BMI is related to attentional approach towards high-calorie food cues (e.g., Castellanos, et al., 2009), whereas other studies suggest attentional avoidance of high-calorie food is related to higher BMI (e.g., Nummenmaa, et al., 2011), and even other studies report no significant association between attention bias for high-calorie food and BMI (e.g., Loeber, et al., 2012).

Some studies suggest that obese individuals, in contrast to healthy weight participants, show an increased approach response to food cues. For example, obese participants initially oriented their attention more often towards food cues than towards non-food cues and maintained their gaze longer on food cues than did healthy weight participants, when they were satiated (Castellanos, et al., 2009). A similar effect has been found in obese versus healthy weight children in a foodStroop colour naming task (Braet \& Crombez, 2003). In addition, recordings of ERPs to food cues suggested that overweight and obese participants had trendsignificant increased attention for food (versus neutral cues) when fed in comparison to fed healthy weight participants (Nijs, Muris, et al., 2010). In addition, another study applying EEG reported that an ERP associated with early attention (the P200 amplitude) was higher in obese than in healthy weight participants during a food Stroop (Nijs, Franken, \& Muris, 2010). Thus, together these studies suggest that obese individuals show increased attention towards food cues (i.e., attentional approach), with more consistent evidence for an enhanced early attentional bias (i.e., initial orientation) in overweight and obese participants, particularly when satiated, in comparison to healthy weight individuals. 


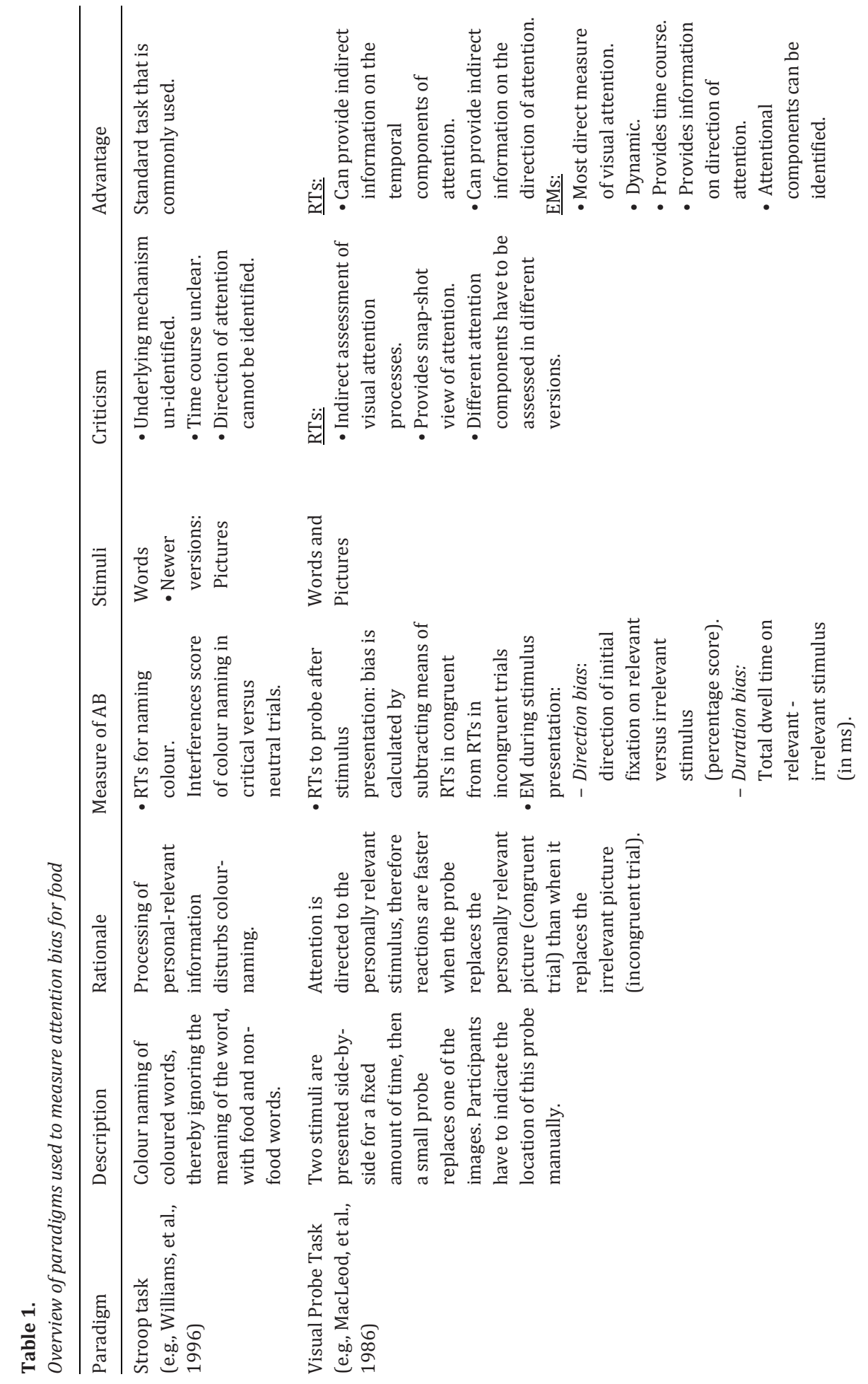




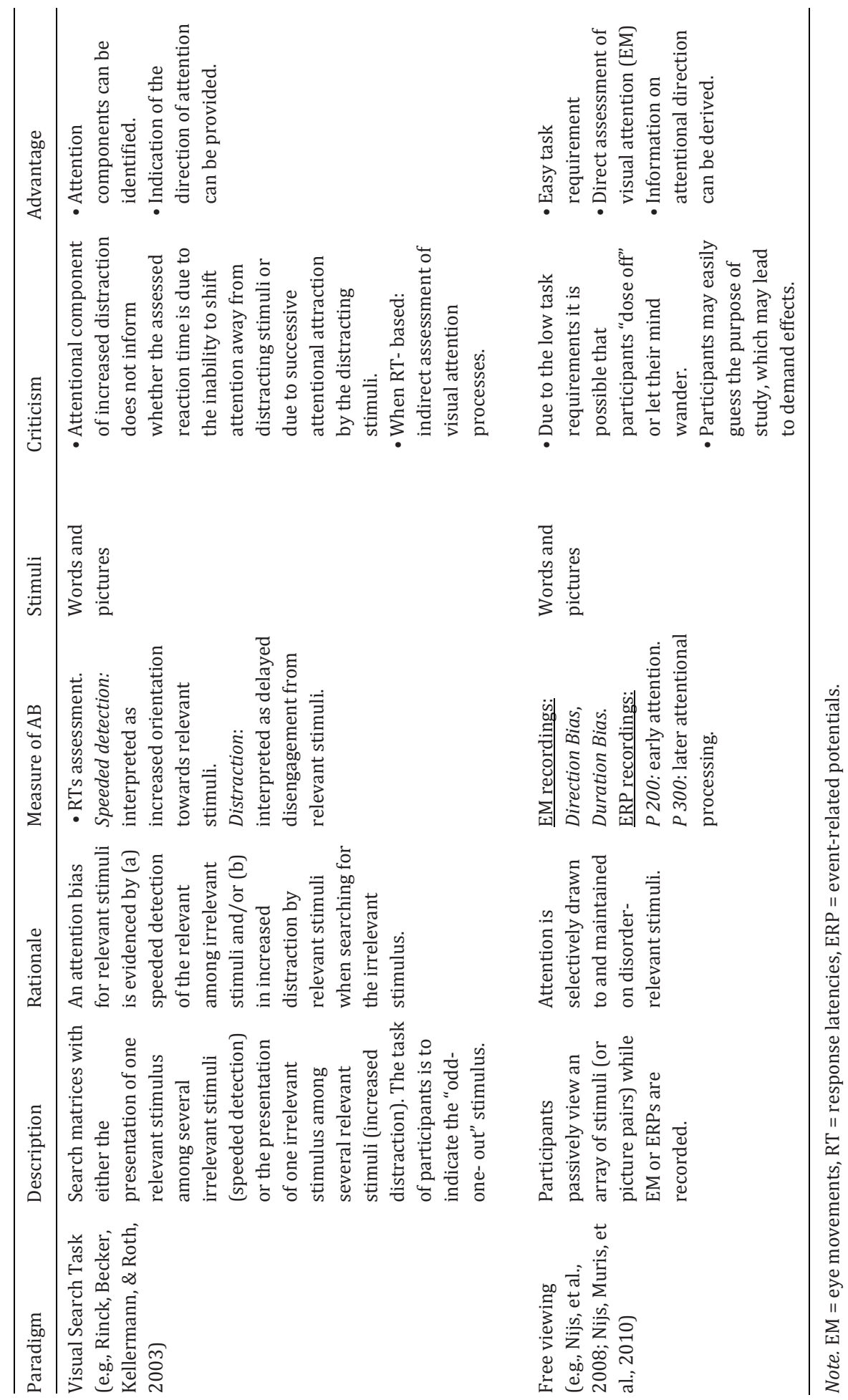


In contrast, other research seems to suggest that a higher BMI is associated with attentional avoidance of food. For example, Nummenmaa and colleagues (2011) reported a negative association between initial orientation for food (irrespective of palatability, as compared to neutral stimuli) and BMI in a group of mainly healthyweight participants (Nummenmaa, et al., 2011). Similarly, healthy-weight participants were found to orientate significantly less often towards low-calorie food than obese participants (with significantly higher dieting intentions). Yet, no differences between weight groups in attention allocation for high-calorie food stimuli were found. Thus, obese participants with dieting intentions paid similar attention to high and low-calorie food stimuli, but healthy weight participants paid significantly less attention to low-calorie food (Graham, Hoover, Ceballos, \& Komogortsev, 2011). Moreover, in obese participants, higher BMI was found to be associated with less initial attention for fried foods (Gearhardt, Treat, Hollingworth, \& Corbin, 2012). Together, these results seem to suggest that a higher BMI is associated with less attention to high-calorie or highly palatable food cues, or with more attention to low-calorie food or even attentional avoidance of high-calorie food types.

However, there are also several studies that did not find a significant impact of BMI on attentional processing of food cues in adults and children (e.g. Loeber, et al., 2012; Pothos, Tapper, \& Calitri, 2009; Soetens \& Braet, 2007). Moreover, some evidence suggests an attention bias for food (versus neutral cues) in healthy weight and obese individuals alike (Nijs, et al., 2008; Nijs, Muris, et al., 2010 see data on eyetracking). With regard to dieting or eating restraint in obese participants, findings are also mixed (see also Chapters 2 and 4). For example, one study found that obese participants, who indicated that they tried to restrain their food intake, had no attentional preference for food cues over neutral cues. In contrast, obese participants who did not restrain their food intake preferentially attended food cues (Nathan et al., 2012, placebo group comparisons). This study suggests that restrained eating reduced attention for food cues. Yet, another study reported that weight loss maintainers had stronger Stroop interference for high-calorie words in comparison to both, obese and healthy weight participants (Phelan, et al., 2011).

Moreover, evidence on the predictive validity of attention bias for high-calorie food on changes in BMI is still lacking. Most conducted studies chose a crosssectional design, leaving interpretations concerning the causal role of attention in the development of overeating, overweight and obesity unresolved, see also Chapters 3 and 7. Only one study showed that food Stroop interference for unhealthy food words was related to BMI increase after one year, whereas interference for healthy food words was related to BMI decrease, in a mostly healthy weight student sample (Calitri, Pothos, Tapper, Brunstrom, \& Rogers, 2010). Table 2 provides an overview of the results and applied methodology of studies that examined foodrelated attention biases in relation to BMI and/or obesity. 


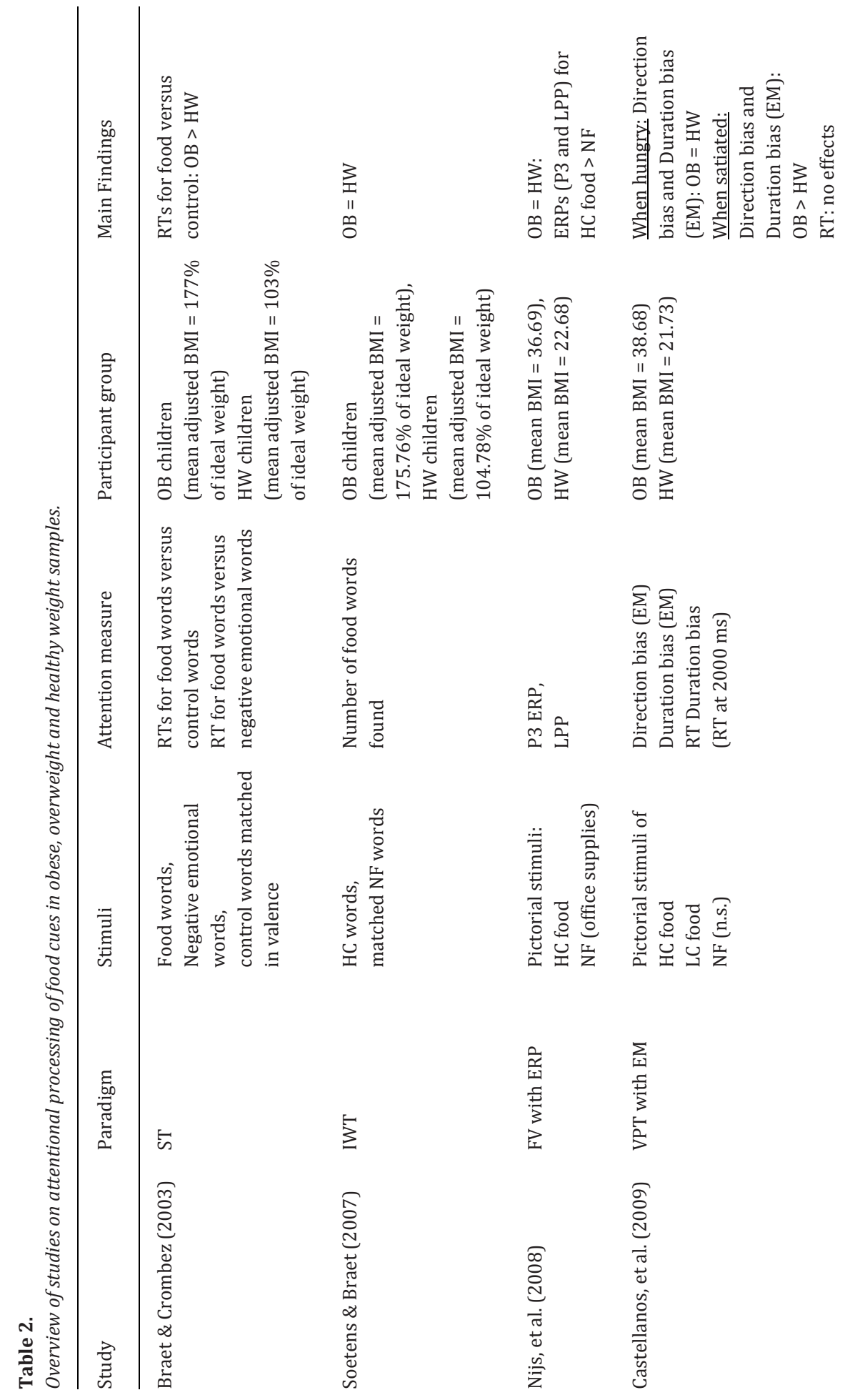




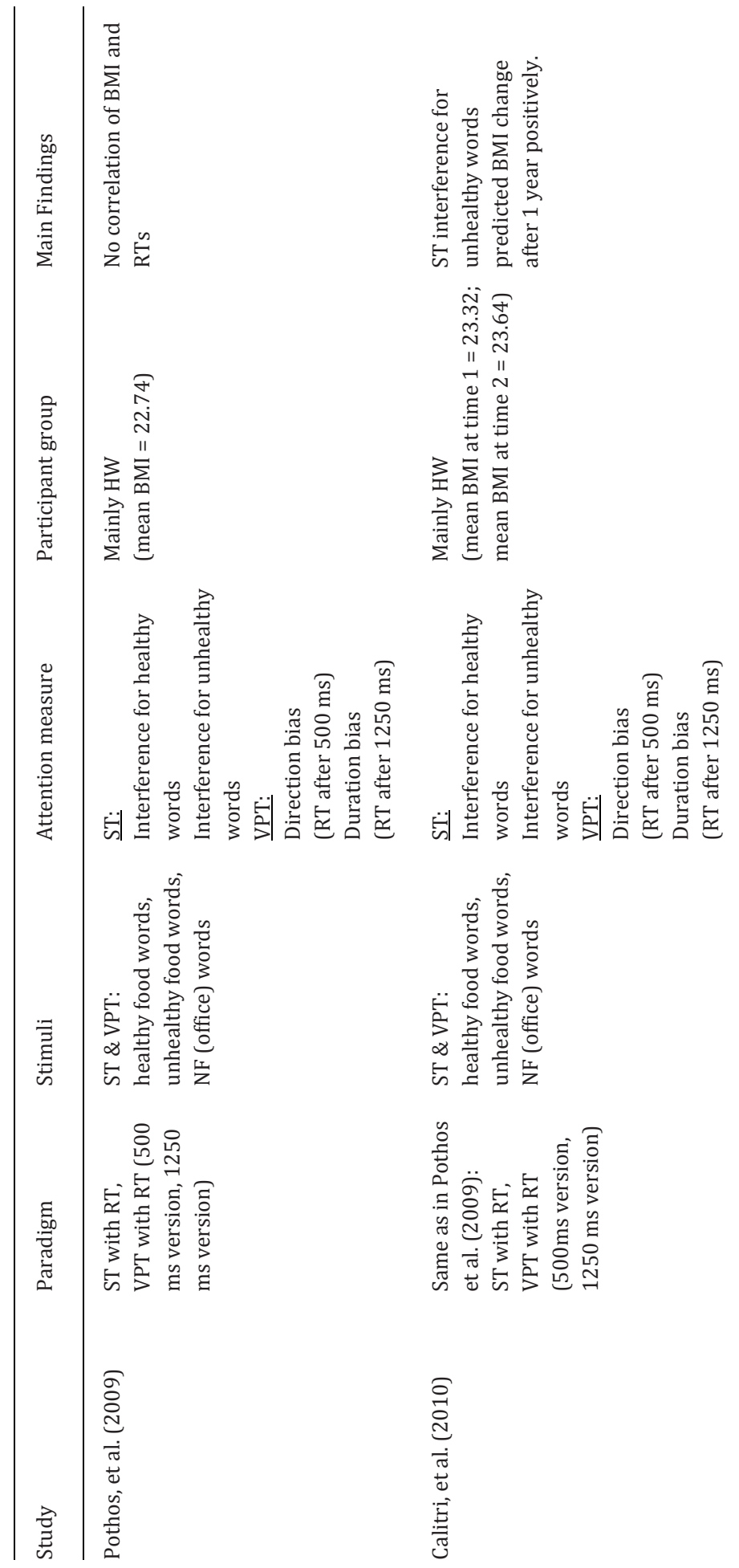




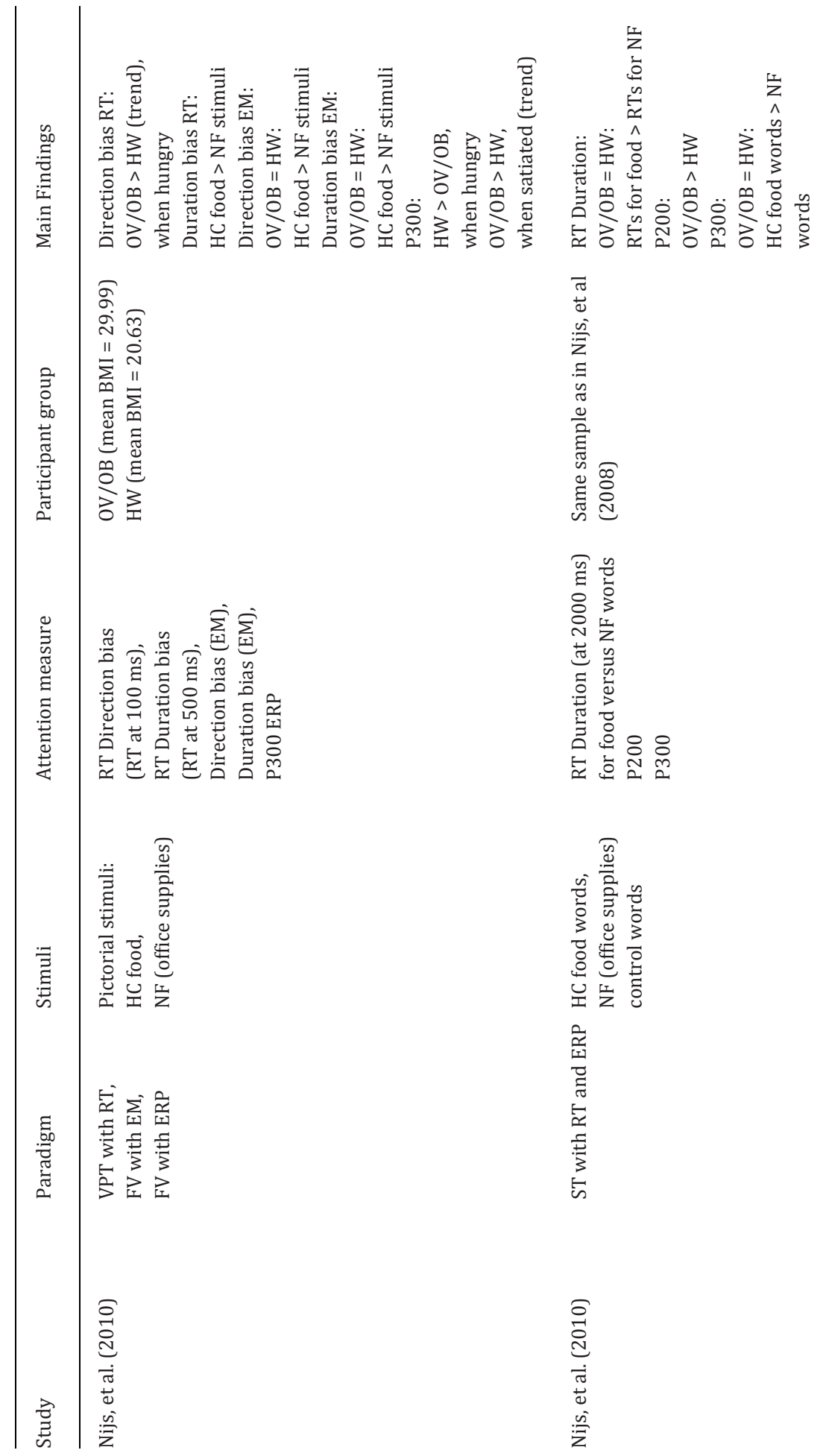




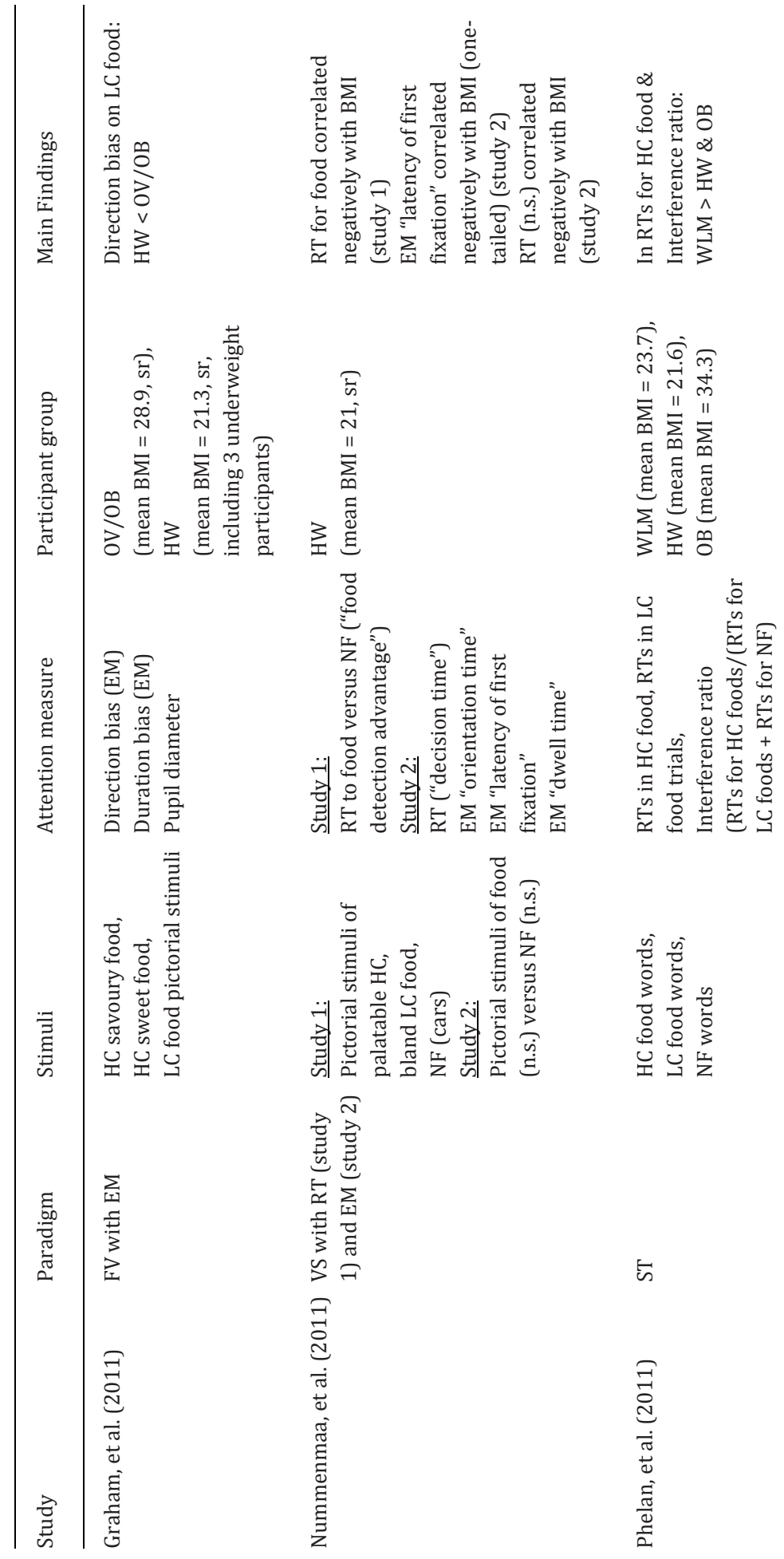




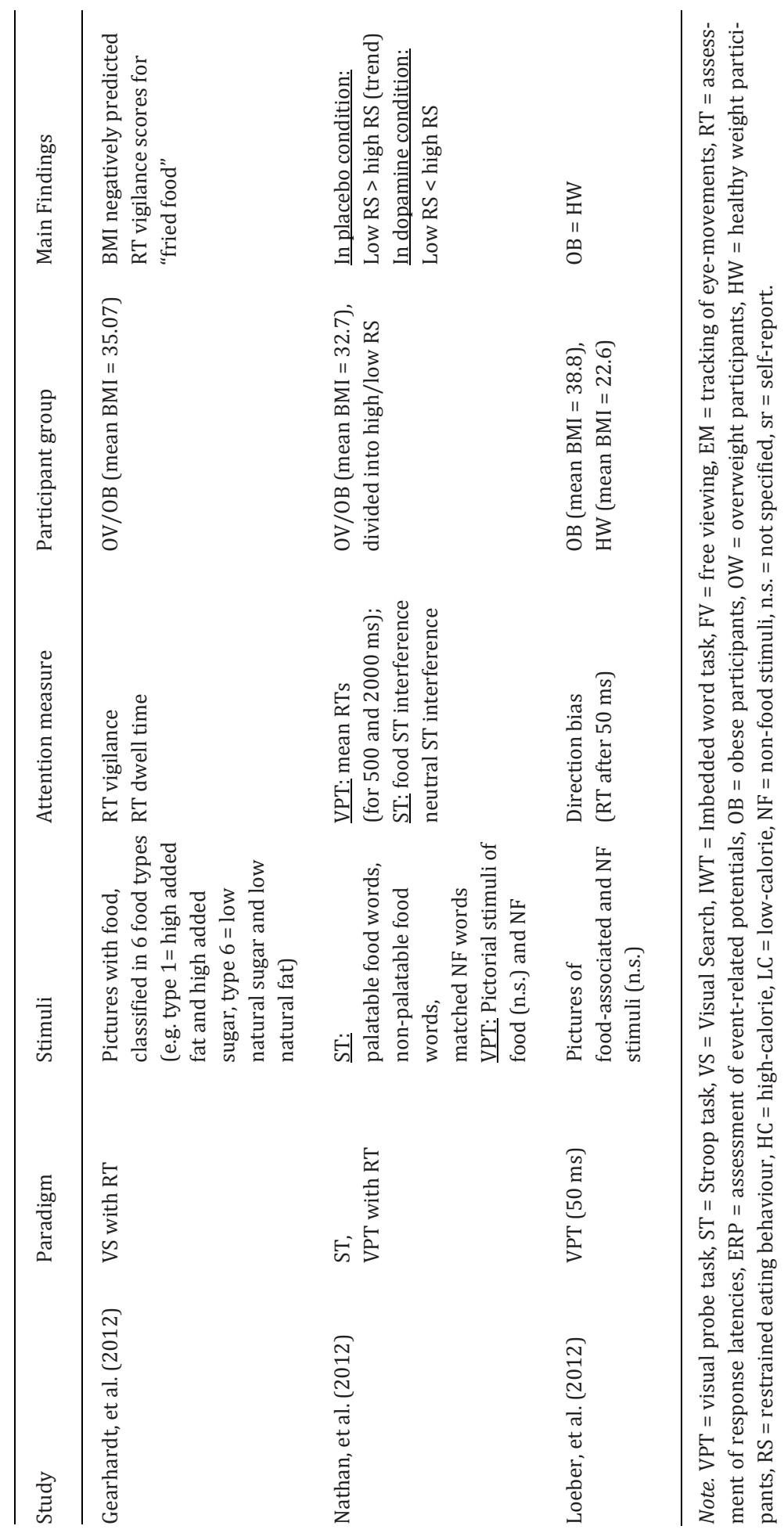




\section{Common pitfalls in previous studies: Does the method determine the result?}

To determine how eating motivation and eating restraint are related to attention bias for food in the context of obesity it is important to use a method that can clearly and directly distinguish between different attentional components (early versus later attention processes) and different attentional mechanisms (approach versus avoidance). Yet, previous studies are inconsistent with regard to the assessment of attention bias (more direct assessment via eye-tracking or EEG recordings versus indirect assessment of response latencies), the components of attention bias that were found to be related to BMI status (early versus later attention processes), the direction of the reported attention bias (attentional approach versus attentional avoidance) and the applied stimuli sets (only food stimuli, food contrasted with neutral stimuli, high-calorie versus low-calorie, healthy versus unhealthy food). However, the accurate distinction in temporal attentional components is important to clarify how differences in eating motivation versus eating restraint are reflected in involuntary attentional processes (early attention) towards food cues versus more controlled attention allocation (attention maintenance) for food. Similarly, it is important to be able to study the direction of attention bias because it has not been systematically investigated if eating restraint contributes to increased attention on "forbidden" food or is related to attentional avoidance of tempting food.

Moreover, stimuli selection might be critical, because it is possible that the contrast category influences the context in which the relevant stimuli is automatically evaluated (see for a similar argumentation when using implicit measures, e.g. Houben, Roefs, \& Jansen, 2010; Roefs et al., 2011; Roefs et al., 2006). For example, by presenting high-calorie food together with low-calorie food, overweight or obese participants might be primed with the concept of "health" whereas when high-calorie stimuli are paired with neutral or non-food stimuli the activation of this association is less likely. Thus, the combination of stimuli could contribute to the observed results. To fully understand how eating motivation versus eating restraint is related to attention bias for food in the context of obesity, it is imminent to go beyond a cross-sectional observation of the relationship of attention for food and BMI to be able to determine the role of attention bias for food on food intake and BMI change.

\section{Outline and hypotheses of the current thesis}

One overarching aim of this thesis is to examine attentional mechanisms and the direction of the attentional processes contributing to an attention bias for food in 
the context of obesity. To this end, my co-authors and I studied the association between BMI and biased attention for food, and extend previous research by using eye-movement registration to gain a detailed understanding of this association (Chapters 2 and 3). Furthermore, we extend previous research by examining whether biased attention for food cues is distinctly related to eating motivation or eating restraint (Chapters 4, 5 and 6). Finally, we conducted experimental and longitudinal studies to shed more light on the causal relationship between attention bias and BMI and food intake (Chapters 3 and 7).

Several methodological choices have been made. First of all, we applied the method of eye-tracking, which is the most sensitive measure of visual attentional processes currently available. We combined recordings of eye-movements with a standard measure of attention bias, the visual probe task (in all studies, except Chapter 7). In addition, we also assessed response latencies during this task to examine how a more direct assessment of visual attention (i.e., eye tracking) relates to the indirect assessment of attentional processing (i.e., response latencies).

Moreover, with regard to our stimulus-sets, in all our studies photographs of high-calorie palatable foods were contrasted with photographs of non-food objects (i.e., musical instruments, shoes or animals). Thus, the attentional bias scores of our studies are based on the comparison of selective attention for high-calorie food versus non-food stimuli. High-calorie, palatable foods were chosen, because these are the food cues that are abundantly available in our environment, especially tempting to eat, yet also posit the largest risk for overeating and weight gain. We thus reasoned that including another food category (e.g. low-calorie or healthy food) could distort a "pure" measure of attention bias for high-calorie food by evoking a health-related evaluation context, as the contrast between high-calorie and low-calorie foods then becomes very obvious to participants.

This thesis presents a series of studies on the relationship between individual differences in BMI and eating motivation or eating restraint on the one hand, and attention bias for food on the other hand. The first part of this thesis focusses on the relationship between BMI and biased attention for food. We hypothesized that higher BMI is related to more attention for food, and that paying more attention towards food cues contributes to weight gain. Specifically, in Chapter 2, differences in attention biases for high-calorie food cues were compared between overweight and healthy weight students. In this study effects of an attention bias for food on craving and eating behaviour have been assessed as well. In the study described in Chapter 3 differences in attention allocation on food cues was compared between healthy weight and severely obese children. In addition, the predictive value of attention biases for food and weight changes in obese children was studied.

The second part of this thesis presents studies investigating the relationship of differences in eating motivation, restrained eating behaviour and mood states on biased attention allocation for food cues. In addition, we also examined the 
relation between food-related attention biases and craving and food intake. We hypothesized that differences in eating-related motivations (for example eating enjoyment versus eating restraint) affect attention mechanisms in different stages of attentional processing (early, thus more automatic versus later, thus more intentional processes) and in the direction of attention (attentional approach versus avoidance). In this respect, the study presented in Chapter 4 examines whether attention allocation for food cues differs between restrained and unrestrained eaters, when testing healthy weight women, as previous research findings were often confounded by BMI differences between those two groups. In the study described in Chapter 5, an experimental manipulation of eating motivation for chocolate has been conducted to understand if and how eating allowance versus eating forbiddance/restraint affects distinct attention allocation patterns for chocolate cues. In addition, differences in craving and eating behaviour were tested and associations with attention biases for chocolate cues were examined. Moreover, in Chapter 6, a study on the influence of an experimental negative versus neutral mood induction on eating behaviour and attention allocation towards high-calorie food cues within a healthy weight sample is illustrated. Again, associations of attention biases and eating behaviour were inspected.

In the last section of this thesis, the causal relationship of attention biases for food and subsequent food intake was tested. We hypothesized that an attention bias for food increases food intake. In Chapter 7, a manipulation of attention for food (towards chocolate and towards shoes) by means of a novel attention modification task (the anti-saccade task) is described within a healthy weight female sample. Eye-movements were monitored to account for the accuracy of the attention modification and craving, chocolate intake and search time for hidden chocolate (as measure of chocolate-related motivation) have been assessed.

In Chapter 8 an overview of the main findings of the studies described in this thesis is presented. In addition, current findings on the association and possible relation of attention allocation processes on food cues and subsequent eating behaviour and weight gain are discussed and a model incorporating previous and current findings on attention bias, BMI, eating motivation and food intake is proposed. 



\section{Chapter 2}

Can(not) take my eyes off it:

Attention bias for food in overweight participants

Published as:

Werthmann, J., Roefs, A., Nederkoorn, C., Mogg, K., Bradley, B. P., \& Jansen, A. (2011). Can(not) take my eyes off it: Attention bias for food in overweight participants. Health Psychology, 30, 561-569. 


\section{Abstract}

Objective: The aim of the current study was to investigate attention biases for food cues, craving and overeating in overweight and healthy weight participants. Specifically, it was tested whether attention allocation processes towards high-fat foods differ between overweight and normal weight individuals and whether selective attention biases for food cues are related to craving and food intake.

Method: Eye movements were recorded as a direct index of attention allocation in a sample of 22 overweight/obese and 29 healthy weight female students during a visual probe task with food pictures. In addition, self-reported craving and actual food intake during a bogus "taste-test" were assessed.

Results: Overweight participants showed an approach-avoidance pattern of attention allocation towards high-fat food: Overweight participants directed their first gaze more often towards food pictures than healthy weight individuals, but subsequently showed reduced maintenance of attention on these pictures. For overweight participants, craving was related to initial orientation towards food. Moreover, overweight participants consumed significantly more snack food than healthy weight participants.

Conclusion: Results emphasize the importance of identifying different attention bias components in overweight individuals with regard to craving and subsequent overeating.

Keywords: Overweight, attention bias, craving, overeating 
On a nutrition level obesity is caused by an imbalance of energy intake and energy expenditure. A crucial factor influencing the development of this imbalance is the "obesogenic"-food environment, which is characterized by an abundance of highly palatable food items. In such an environment, people are frequently confronted with attractive food cues, leading to a persistent temptation to indulge (Hill \& Peters, 1998; Lowe \& Levine, 2005). High-fat foods are particularly problematic because they are highly palatable and energy-dense (Polivy, Herman, \& Coelho, 2008; Schrauwen \& Westerterp, 2000). Moreover, high-fat foods are overly represented in the visual food environment because most of these foods are heavily advertised (Hoek \& Gendall, 2006). However, not everyone is equally susceptible to the constant temptations of high-fat foods in our environment (Polivy, et al., 2008; Tetley, Brunstrom, \& Griffiths, 2009). Recent research (e.g. Davis et al., 2007; Giesen, Havermans, Douven, Tekelenburg, \& Jansen, 2010; Stice, Spoor, Ng, \& Zald, 2009) suggests that overweight and obese individuals find these foods particularly rewarding. Because high-fat foods are more rewarding, these people may selectively attend to these high-fat foods. Vice versa, a tendency to automatically notice and attend to high-fat foods may contribute to the maintenance of craving for these foods.

It has been proposed that attention biases for cues follow from attributing incentive salience to the cues (e.g. Berridge, 2009). As a consequence of a conditioning process, that is, repeated associations between food cues and a rewarding experience, the cues (e.g., the sight of palatable high-fat foods) become salient and attract attention. The food cues gain "attention grabbing powers", which enable them to capture attention, trigger or elevate craving and elicit approach behaviour towards them (Berridge, 2009; Field \& Cox, 2008; Field, Munafó, \& Franken, 2009; Franken, 2003).

Craving, in turn, plays a central role in promoting consumption and challenging control over intake (Martin, O'Neil, Tollefson, Greenway, \& White, 2008; Tiffany, 1999). It is assumed that attention biases and craving are reciprocally related, that is, attention biases for high-fat food may elicit craving, while craving can in turn trigger attention biases for high-fat food (Field \& Cox, 2008; Field, et al., 2009; e.g. Ryan, 2002; Smeets, Roefs, \& Jansen, 2009). This self-perpetuating circle might, in the long run, lead to a steady preoccupation with the desired stimuli (Franken, 2003). The aim of this study is therefore to investigate whether the attention-grabbing potential of high-fat food cues differs between overweight and healthy weight participants. It is also studied whether selective attention biases for high-fat foods are related to increased craving and food consumption.

Attention biases for motivational salient cues have already been extensively studied in the field of addiction disorders. A bias in initial orientation to addictionrelated cues, which might reflect a rapid detection or vigilance mechanism, has often been observed in addiction disorders, such as nicotine and alcohol depend- 
ence (Bradley, Mogg, Wright, \& Field, 2003; Field \& Cox, 2008). Attention maintenance on relevant addiction-related stimuli (i.e. prolonged holding of attention, after initial orientation) has also been associated with craving and approach behaviour towards desired drug stimuli in some addiction research, e.g. young adult cigarette smokers (Field, Mogg, \& Bradley, 2004; Mogg, Field, \& Bradley, 2005). However, biases in attention maintenance can be susceptible to controlled avoidance strategies; i.e., although attention may be initially directly towards reward cues (e.g. desired stimuli), some individuals may subsequently try to reduce maintained attention on them; for example, if they are actively attempting to suppress craving (Field \& Cox, 2008). Given that addiction and overeating may share common cognitive and neural mechanisms (Davis \& Carter, 2009) we expected biases in both initial orientation and attention maintenance components to be influential factors for craving and subsequent intake of high-fat foods.

Research on attention processes in participants with overweight and obesity is relatively sparse and yielded contradictory results. On a Stroop task, obese children showed greater interference in colour-naming food words than did their lean counterparts (Braet \& Crombez, 2003). However, another study, using the same paradigm, reported no significant differences between obese and healthy-weight adults (Phelan et al., 2010). Moreover, a study using a letter grid with hidden food words (i.e. the imbedded food word task), also failed to find interference for food words in overweight adolescences (Soetens \& Braet, 2007).

Also studies in which attention was directly assessed by means of eyemovement registration yielded contradictory results. A recent study (Castellanos et al., 2009), measuring eye movements during a visual probe task with food and non-food pictures, found that obese participants, in contrast to healthy-weight participants, demonstrated enhanced initial orientation towards and maintained attention on food cues. In contrast, another study that assessed eye-movements to food pictures in a passive viewing task did not find a bias in eye movement recording between obese and healthy weight adults (Nijs, Muris, Euser, \& Franken, 2010). However, in this same study participants also completed a visual probe task with manual response latencies as a dependent measure. In this task, overweight participants demonstrated a bias in enhanced initial orientation towards food cues, but showed no bias in maintained attention (all participants, irrespective of weight, showed maintained attention on food in contrast to neutral cues). Furthermore, results from a separate attentional task assessing event-related potentials (ERP, P300) suggested that some overweight participants may have used "cognitive strategies to reduce a maintained attentional bias for food stimuli" (Nijs, et al., 2010).

Whereas the findings by Castellanos et al. (2009) and Nijs et al. (2010) support the existence of the hypothesized attentional bias in initial orientation towards food cues in overweight participants, research into biases in maintained 
attention, such as the study by Nijs et al. (2010) showed mixed results, possibly depending on their paradigms. In addition, the studies by Phelan et al. (2010) and Soetens \& Braet (2007) yielded no differences in attentional biases for foodrelated words between overweight and healthy-weight participants, although (Braet \& Crombez, 2003) found such a difference in a modified Stroop study. It is possible that the inconsistent results of earlier studies can be explained by different methodologies that were applied to study attention allocation.

Indirect measures of attention, such as the Stroop paradigm, are difficult to interpret because it is not possible to disentangle distinctive attentional processes, that is, initial attention allocation or maintained attention. The most direct way to assess attention allocation processes is to record eye movements during a visual attention paradigm. Recording of eye movements provides a direct observable, dynamic and ecologically valid measure of visual attention processes (Mogg, Bradley, Field, \& De Houwer, 2003). Another key advantage of eye movement recording is that it is easy to accurately disentangle distinctive components of attention allocation processes, such as initial orientation and attention maintenance. Hence, we opted to employ eye-tracking during a visual probe task with food cues versus neutral cues in the current study. This direct assessment of distinctive components of attention allocation towards food might further contribute to clarify divergent results of previous studies. We further aim to extend previous findings by examining the relationships between specific attention bias components (initial orientation, attention maintenance) and craving and (over)eating. This might enable us to deduce at which stages of information processing overweight individuals differ from healthy weight individuals when exposed to food cues and how these processes are related to eating behavior that is associated with the development of obesity.

To summarize, the current study examined attentional biases towards high-fat food in overweight and healthy-weight participants. It was hypothesized, on both theoretical and empirical grounds, that overweight participants would show a larger attentional bias in initial orientation (i.e. enhanced initial gaze direction) towards food cues as compared to healthy-weight control participants. In addition, we expected overweight and normal-weight participants to differ in their subsequent maintenance of gaze on food cues relative to non-food cues, manifesting in both recordings of eye movements and recordings of manual response latencies during the visual probe task. The latter prediction was not directional, as overweight individuals may show either increased or reduced maintenance of attention on food cues, as discussed earlier (Castellanos et al., 2009; Nijs et al., 2010). Moreover, the hypothesis was tested that overweight participants would eat more snack food than normal-weight individuals on a bogus 'taste-test'. We also examined whether attentional biases for food cues are related to reports of food-craving 
(assessed immediately before and after the attentional task) and increased food intake.

\section{Method}

Sample and participant selection

The sample comprised 22 overweight/obese and 29 healthy-weight female students. Participants were selected for the study according to their body mass index (BMI; $\mathrm{kg} / \mathrm{m}^{2}$ ) based on their self-reported height and weight in a survey among first-year students (BMI < 25 for healthy-weight participants and BMI $>25$ for overweight/obese participants). Exclusion criteria were serious health problems, pregnancy and vegetarism, because food stimuli included pictures of meat. Final allocation of participants to the overweight/obese and healthy-weight groups was based on BMI measures obtained from accurate height and weight measurements taken at the end of the test session. See Table 1 for sample characteristics.

\section{Materials}

\section{Pictorial visual probe paradigm}

Overview. A visual probe paradigm was employed, with concurrent recording of eye movements as a direct measure of attention allocation, and the assessment of response latencies as an indirect index of attention allocation. In the visual probe paradigm, image pairs were presented simultaneously side by side, followed by the presentation of a probe appearing in the location of one of the images. Participants were instructed to respond as quickly as possible by pressing a corresponding key on a button box to indicate the location of the probe. The logic of this task presumes that participants are faster in detecting probes appearing in the location of the stimulus that they attended. Conversely, it is assumed that participants are slower to respond to probes appearing in the location of the stimulus that they did not attend (MacLeod, Mathews, \& Tata, 1986).

Timing Trials. Each trial started with a central fixation cross, which disappeared directly after participants fixated on it. Subsequently, the target image pair was presented for $2000 \mathrm{~ms}$. Then the probe $\left(^{*}\right)$, was presented until the participant responded by pressing the appropriate key on a button box.

Trial types. The visual probe paradigm included 120 trials: 80 critical trials and 40 filler trials. Critical trials consisted of 20 stimulus pairs, which were each presented four times. Filler trials consisted of 10 stimulus pairs, which were also presented four times. The position of the probe was equally distributed per stimu- 
lus type and was also equally distributed over the left and the right side of the screen. The order of trials was randomized uniquely for each participant.

Stimuli. Stimuli were naturalistic images of highly palatable food items because food words might have weaker motivational effects (Simmons, Martin, \& Barsalou, 2005; Tiggemann \& Kemps, 2005). In critical trials, the image pair consisted of a picture of a palatable high-fat food item and a picture of a musical instrument. Filler trials consisted of picture pairs depicting two neutral non-food pictures (office supplies and traffic objects). All image pairs were matched as closely as possible with regard to colour, complexity and brightness, and size. Each picture was presented equally often on the left and on the right side of the screen.

Eye movement measurements. The visual probe paradigm was run on a Dell OptiPlex $^{\text {TM }} 760$ Computer with a 19-inch screen (resolution: $1280 \times 1024$ ). Participants were seated within approximately $50 \mathrm{~cm}$ distance to the screen in a dimly lit room. Eye movements were recorded by a desktop mounted EyeLink 1000 system (SR Research Ltd., Mississauga, Ontario, Canada). A 9-point calibration with subsequent validation procedure was conducted prior to the visual probe paradigm. To study attention allocation processes, participants' gaze fixations were examined. Gaze fixations were defined as any period that was not a blink or saccade and lasted at least 100 ms (Eyelink Dataviewer User's Manual, 2002-2008, SR Research Ltd.). Eye movements that occurred before the presentation of an image pair were excluded, because these movements could represent anticipatory fixations.

For analyses purposes, the computer screen was, invisible for participants, divided into three areas of interest: the mid-section, which represented the location of the fixation cross, and the left and right section, representing the locations of the picture stimuli. Only eye movements in critical trials directed either to the left or the right section of the screen were extracted for further analyses. Eye movements in filler trials and gaze fixations in the mid area were excluded from further analyses. Eye movements were extracted using Data Viewer (SR Research Ltd., Missisauga, Ontario, Canada).

Attention Bias Scores. Three attention bias scores were derived from the eye movement data: gaze direction bias scores, initial fixation duration bias scores and gaze dwell time bias scores.

The gaze direction bias was based on the proportion of trials on which the first fixation was directed to a food stimulus versus a control stimulus. A fixation was defined as a first fixation on a picture if it was indeed the first fixation in that trial, but also if it was preceded by a fixation in the mid area. Following Castellanos et al. (2009), direction bias scores were calculated by computing the number of first fixations that were directed to a high-fat food picture as a proportion of all trials on which a first fixation was made to either picture. A bias score greater than $50 \%$ represents a higher proportion of first fixations directed to food stimuli, 
whereas a bias score less than 50\% indicates a higher proportion of first fixations directed to non-food stimuli.

The initial fixation duration bias is based on the duration of the first fixation on a critical stimulus and can be seen as a measure for early attention maintenance (e.g. Mogg, et al., 2003). The initial fixation on a picture was defined as the first to occur following the onset of the picture pair. Thus, this variable represents the duration of a first fixation that is directed to one of the picture stimuli (food vs. non-food). Initial fixation durations per image category (food or non-food) were averaged over the relevant trials per participant. Bias scores for the initial fixation duration were computed by subtracting the mean duration of initial fixations directed to non-food images from the mean duration of initial fixations directed to food images. Thus, a positive score is indicative of longer initial attention maintenance on food stimuli, whereas a negative score is indicative of the reverse: longer initial maintenance on non-food stimuli.

Gaze dwell time is informative regarding the maintenance of attention on critical stimuli (e.g. Mogg, et al., 2005). Overall dwell time per image category (food vs. non-food) was summed over individual fixations across each critical trial, and then averaged per image category over all trials, resulting in an average total dwell time per image category for each participant. For the gaze dwell time bias score, the mean dwell time on non-food images was subtracted from the mean dwell time on food images. Thus, a positive score indicates that attention was maintained longer on food items than on non-food items, whereas a negative score indicates the reverse: longer maintained attention on non-food items.

Manual response latencies to probes. Calculations of response latency bias scores were based on the recordings of the participant's manual response latency when indicating the location of the probe. Response latencies were excluded from further analyses if they were faster than $200 \mathrm{~ms}$, slower than $2000 \mathrm{~ms}$, and then if they deviated more than 3 SDs from each participant's mean (e.g. Castellanos, et al., 2009; Mogg, Bradley, Hyare, \& Lee, 1998). Response latency bias scores were then calculated by subtracting the mean response latency on congruent trials (that is, when the probe replaced a food image) from the mean response latency on incongruent trials (that is, when the probe appeared in the same location as the preceding non-food image). A positive bias score indicates an attention bias towards food, whereas a negative bias score indicates an attention bias away from food.

\section{Questionnaires}

Craving \& Satiety. Craving was assessed by asking "How strong is your craving for food right now" on a $100 \mathrm{~mm}$ visual analogue scale (VAS), ranging from "no craving at all" (0) to "extremely strong craving" (100). As adjunct to craving, satiety was also assessed by three $100 \mathrm{~mm}$ VAS addressing the experience of hunger and satiety. The satiety-VAS ranged from 0 , indicating an absolute absence of hun- 
ger/satiety, to 100 , indicating an overwhelming presence of hunger/satiety. The VAS was originally derived as a "gold standard" method to assess pain, and has recently been validated as an adequate instrument to assess appetite (see for a discussion Flint, Raben, Blundell, \& Astrup, 2000).

Body Mass Index (BMI). BMI was assessed at the end of the experimental session. Participants' weight (in $\mathrm{kg}$ ) and height (in $\mathrm{m}$ ) was measured accurately and BMI was calculated.

Taste Test. Food consumption was measured by means of a bogus "taste test". Participants were instructed to rate four bowls (mean weight (in grams) = $1218.37, \mathrm{SD}=89.52$ ) of highly palatable fat food items (chocolate, biscuits, crisps, salted peanuts) in terms of their visual attractiveness, smell, and taste. The participant was given 10 minutes to complete her ratings and was told that she was free to try as much of the offered items as she liked. Consumption was determined by weighing the bowls both before and after the "taste test" and the difference in weight from pre to post assessment was converted into calories and used as a measure of food intake.

\section{Procedure}

The study was reviewed and approved by the local Ethics Committee. Participants were asked to refrain from eating two hours prior to their laboratory appointment, which was scheduled at lunch time. Previous studies indicated that hunger may obscure differences between overweight and healthy participants because being hungry elevates attention focus and craving for food independent of weight status (Castellanos, et al., 2009; Mogg, et al., 1998; Nijs, et al., 2010; Piech, Pastorino, \& Zald, 2010). Therefore, in the current study we made sure all participants were equally satiated, by providing them with a lunch before assessing eye movements during the visual probe task. Upon arrival, participants received a lunch consisting of the participant's choice of a sandwich (cheese or ham on brown bread rolls) and their choice of a yoghurt or juice drink. The lunch contained in total approximately 400-500 calories, in accordance with the average calorie value of lunches of Dutch students (deCastro, Bellisle, Feunekes, Dalix, \& DeGraaf, 1997). After the lunch, the participant filled in the craving-VAS and the satiety-VAS (time 1). Subsequently she was asked to take a break for 20 minutes to ensure that satiety-related hormones signalled satiety to the brain (e.g. Castellanos, et al., 2009; Nijs, et al., 2010). After the break, the participant answered the craving-VAS and the satiety-VAS again (time 2) and then performed the visual probe paradigm. Afterwards, she again completed the craving-VAS and the satiety-VAS (time 3). Then the participant completed another computer task that is not relevant for the current study, which lasted for 15 minutes. Subsequently the bogus taste test was arranged. Shortly before the taste test the participant filled in the craving-VAS and satiety-VAS again (time 4) and was afterwards presented with the bogus taste test. Following the 
taste test, the participant filled in several questionnaires about eating behaviour and current affective state. These questionnaires were included to examine whether groups differed on other state and trait aspects that might be relevant for attention biases for food, such as restrained eating (Restraint Scale; Herman \& Polivy, 1980) hedonic hunger (Power of Food Scale; Lowe et al., 2009), external eating (10 items, included in the Dutch Eating Behavior Questionnaire; van Strien, Frijters, Bergers, \& Defares, 1986) and negative versus positive affect (PANAS; Watson, Clark, \& Tellegen, 1988). At the end of the experimental session, the participant's weight and height were measured. The participant was probed for suspicion and was asked to write down her thoughts on the purpose of the experiment. Finally the participant was thanked for her participation and received either one course credit or a voucher of €7.50.

\section{Results}

\section{Sample characteristics}

Overweight and healthy-weight participants differed with regard to their restrained eating style $(t(49)=4.92, p<.001)$. No other significant group differences in self-reported eating behavior or current affect were observed, see Table 1 for further group details. Five additional participants were tested but were excluded from analyses because they were identified as outliers: three participants deviated in their food consumption more than two SD from their group mean. Three participants did not move their eyes on a sufficient proportion $(<50 \%)$ of trials during the visual probe paradigm (Bradley, Mogg, \& Millar, 2000), one of whom also consumed more than two SDs above her group mean.

\section{Eye-movements measures}

The final sample of participants made eye movements on an average of $90.4 \%$ (SD $=8.73$ ) of all 120 trials. Percentage of eye movements did not differ significantly between overweight and healthy-weight participants, $t(46)=0.26, p=.76$. Levene's test indicated unequal variances $(F=6.00, p=.02)$, so degrees of freedom were adjusted from 49 to 46 .

\section{Attention bias measures}

To test our hypotheses regarding attention biases, independent samples t-tests were conducted with weight status (BMI > 25: overweight; BMI < 25: healthyweight) as the between group factor and attention bias scores as the dependent variables. Alpha level was set to .05, two-tailed, unless otherwise specified. For the mean attention bias scores, see Table 2 and Figure 1. 
Table 1.

Participants' characteristics.

\begin{tabular}{|c|c|c|c|c|c|c|c|c|}
\hline \multirow[b]{2}{*}{ Variable } & \multicolumn{3}{|c|}{$\begin{array}{l}\text { Overweight/Obese } \\
(\mathrm{n}=22)\end{array}$} & \multicolumn{3}{|c|}{$\begin{array}{l}\text { Healthy weight } \\
(\mathrm{n}=29)\end{array}$} & \multirow[t]{2}{*}{$t(49)$} & \multirow[t]{2}{*}{$p$} \\
\hline & $M$ & $S D$ & Range & $M$ & $S D$ & Range & & \\
\hline Age & 19.86 & 1.28 & $18-23$ & 19.31 & 1.95 & $18-27$ & 1.15 & .25 \\
\hline BMI & 28.03 & 3.74 & $25.09-40.04$ & 21.16 & 2.03 & $17.83-24.87$ & 8.39 & $<.001$ \\
\hline RS & 14.91 & 4.31 & $7-21$ & 8.70 & 4.57 & $1-21$ & 4.92 & $<.001$ \\
\hline DEBQ-EX & 2.83 & 0.49 & $1.80-3.70$ & 2.98 & 0.59 & $1.90-4.50$ & 0.94 & .35 \\
\hline PFS & 2.36 & 0.68 & $1.07-3.67$ & 2.20 & 0.64 & $1.07-4.00$ & 0.84 & .41 \\
\hline $\begin{array}{l}\text { Negative Affect } \\
\text { (PANAS) }\end{array}$ & 19.73 & 6.87 & $10-35$ & 20.41 & 5.80 & $11-31$ & 0.39 & .70 \\
\hline $\begin{array}{l}\text { Positive Affect } \\
\text { (PANAS) }\end{array}$ & 35.32 & 5.84 & $24-50$ & 34.21 & 6.10 & $21-44$ & 0.66 & .52 \\
\hline
\end{tabular}

Note. BMI = Body Mass Index, RS = Restraint Scale (Herman \& Polivy, 1980), DEBQ-EX = Dutch Eating Behavior Questionnaire-External Eating subscale (van Strien, et al., 1986), PFS = Power of Food Scale (Lowe, et al., 2009), PANAS = Positive and Negative Affect Scale (Watson, et al., 1988).

Table 2.

Mean attention bias scores and mean food intake (in calories) for overweight/obese and healthy weight participants, respectively.

\begin{tabular}{|c|c|c|c|c|}
\hline \multirow[b]{2}{*}{ Variable } & \multicolumn{2}{|c|}{$\begin{array}{l}\text { Overweight/Obese } \\
(\mathrm{n}=22)\end{array}$} & \multicolumn{2}{|c|}{$\begin{array}{l}\text { Healthy weight } \\
(\mathrm{n}=29)\end{array}$} \\
\hline & $M$ & $S D$ & $M$ & $S D$ \\
\hline \multicolumn{5}{|l|}{ Attention Bias Scores } \\
\hline Gaze direction bias (\%) & 53.70 & 5.00 & 51.33 & 4.20 \\
\hline Initial fixation duration bias (ms) & -15.59 & 51.91 & 14.29 & 46.03 \\
\hline Gaze dwell time bias (ms) & -2.33 & 152.13 & 8.09 & 139.35 \\
\hline Response latenc bias (ms) & -7.22 & 20.67 & 0.76 & 21.18 \\
\hline \multicolumn{5}{|l|}{ Food intake } \\
\hline Calories consumed & 165.92 & 97.15 & 119.95 & 71.63 \\
\hline
\end{tabular}

Note. Gaze direction bias = $\mathrm{N}$ of first fixations on high-fat food stimuli / $\mathrm{N}$ of first fixations on high-fat food stimuli $+\mathrm{N}$ of first fixations on non-food stimuli) $* 100$. Initial fixation duration bias $=$ mean initial fixation duration on high-fat food stimuli - mean initial fixation duration on non-food stimuli). Gaze dwell time bias $=$ mean total dwell time on high-fat food stimuli - mean total dwell time on non-food stimuli. 
Gaze direction bias. In line with our hypothesis, it was found that overweight participants directed their first fixation more often to food images than to non-food images as compared to healthy-weight participants, $t(49)=1.83, p<.05$, onetailed, $d=.52$.

Initial fixation duration bias. Overweight participants had significantly shorter initial gaze fixation durations when they fixated their first gaze on food images than when they fixated their first gaze on non-food images as compared to healthy-weight participants, $t(49)=2.17, p<.05, d=.62$.

Gaze dwell time bias. This bias-score did not differ significantly between the overweight and healthy-weight groups, $t(49)=0.25, p=.80$.

Probe manual response latency bias. This bias-score did not differ significantly between the overweight and healthy-weight groups, $t(49)=1.35, p=.18$.

A

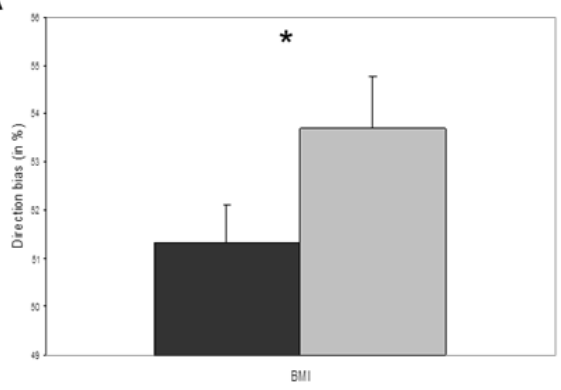

C

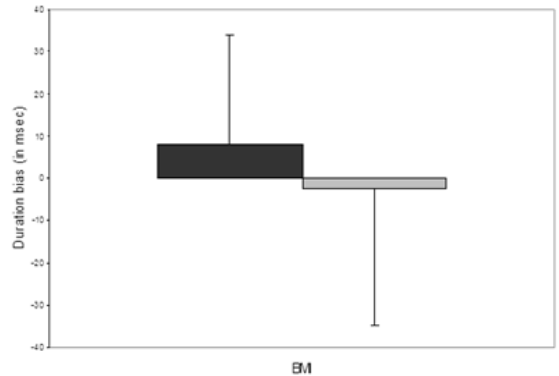

B

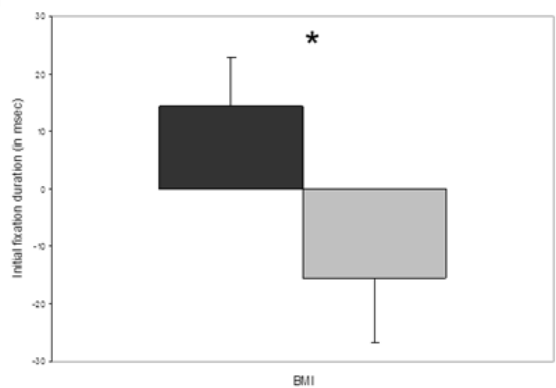

D

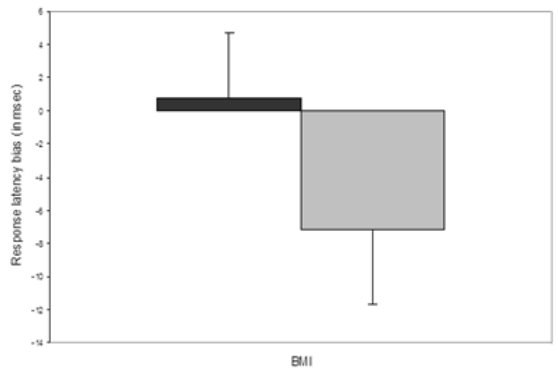

Figure 1. Graph of the mean attention bias scores (with standard errors) per condition (healthy weight/overweight) and bias type. Quadrant A presents mean gaze direction bias scores per group. Quadrant B depicts mean initial fixation duration bias scores per group. Quadrant C shows mean gaze dwell time bias scores per group. Quadrant D displays manual response latency bias scores per group. 


\section{Attention bias, craving and overeating}

Food consumption. In line with our hypothesis, overweight participants ate significantly more calories of snack food during the bogus taste test than did healthy-weight participants, $t(38)=1.87, p<.05$, one-tailed, $d=.61$. Levene's test indicated unequal variances $(F=6.45, p=.01)$, so degrees of freedom were adjusted from 49 to 38.

Craving. Self-reported craving did not differ between groups at any time point, all $t(49)<1.20$, all $p s>.23$. Similarly, there were no significant differences observed on satiety levels between groups at any time point, all $t(49)<1.23$, all $p$ s $>.22$.

Correlation analyses. Bivariate correlations among the two measures of attention biases that revealed significant differences between groups (i.e., gaze direction bias and initial fixation duration bias), and self-reported craving shortly prior (time 2) and directly after (time 3) the visual probe task, and food intake were conducted. Correlations were computed for the group as a whole and for the overweight and healthy-weight groups separately. The results of these correlation analyses were corrected for multiple testing by adjusting the $p$-value. For the correlational analyses with craving the p-values were multiplied by 4 ( 2 attentional bias scores * 2 measures of craving) and for the analyses with food intake the pvalues were multiplied by 2 ( 2 attentional bias scores). A positive relationship of self-reported craving prior to the visual probe task (time 2) and gaze direction bias scores was found uniquely for the overweight group $\ln (20)=.55$, corrected $p=$ .032). No other significant correlations of attention biases and craving or food consumption were found.

\section{Discussion}

The aim of this study was to examine whether specific attention allocation processes towards high-fat food distinguish overweight from healthy-weight participants, and how attentional biases for high-fat food relate to craving and subsequent food consumption across these groups. Our key findings were that, in comparison with healthy-weight individuals, overweight participants showed (i) more frequent initial orientations towards high- fat food, but (ii) diminished initial fixation durations (i.e. after initial orienting, they showed reduced holding of attention on food cues). In addition, (iii) overweight individuals consumed more snack food on a subsequent taste test than did healthy-weight participants and, among overweight individuals: (iv) craving was positively associated with the initial orientation direction bias towards high-fat food.

As compared to healthy-weight participants, overweight participants directed their initial gaze more often towards high-fat food pictures during the visual probe 
task. This is in line with our hypothesis and consistent with findings of previous studies observing a heightened vigilance of food cues in overweight and obese participants (Castellanos, et al., 2009; Nijs, et al., 2010). Contrary to this bias in initial orientation towards food cues, overweight participants showed significantly reduced initial fixation durations for food, which suggests a relatively rapid and immediate attentional shift away from food stimuli. This finding seems unexpected in relation to previous research that reported increased maintained attention (reflected by prolonged gaze dwell time) for food cues in obese participants (Castellanos, et al., 2009). Instead, the present finding seems more compatible with other research evidence (using ERP P300) which suggested that some overweight individuals have reduced maintained attention on food cues (Nijs, et al., 2010).

On the gaze dwell time bias (which was assessed over the entire trial duration) no differences between groups were found. It is possible that the absence of an attention maintenance bias in overweight participants reflects a strategy to avoid focussing for a longer time at food. While the current study, testing mainly overweight participants, failed to find this gaze dwell time bias (which is similar to non-significant gaze dwell time results in Nijs et al., 2010), others have reported increased gaze dwell time bias for food cues in heavily obese participants (Castellanos, et al., 2009). Thus, another explanation for the absence of this bias is that the attention maintenance bias for food might be specific for obese individuals.

Analyses of manual response latencies did not reveal differences between overweight and healthy-weight participants regarding their reactions to congruent versus incongruent trials. The absence of a significant effect might be attributable to the relatively long stimulus duration of $2000 \mathrm{~ms}$. In the traditional visual-probe task, such long stimulus duration are used to examine attention maintenance. Findings of eye-movement recordings, however, showed that group difference emerged only at an early stage of attention allocation, namely in the initial fixation phase. The non-significant difference in response latencies suggest that groups did not differ in their attention allocation at a later phase of recordings. This result highlights the advantage of recording eye movements in addition to recording manual response latencies, because the latter are an indirect and relatively limited measure of attention processes (Field, et al., 2009), as, in the present study, they only assessed attention bias at one specific point in time (i.e. $2000 \mathrm{~ms}$ after picture onset).

Overall, the observed pattern of attention allocation in the present study resembles an approach-avoidance reaction towards food in overweight participants. The approach component presumably arises because food cues are more salient for overweight participants than for healthy-weight participants, possibly reflecting a greater motivational value of these foods (Berridge, 1996). The avoidance component might reflect negative associations with the consequences of indul- 
gence, such as gaining further weight, feelings of guilt and shame, unhealthy nutrition properties and the stigma of being overweight (Fairburn et al., 2008; Macht, Gerer, \& Ellgring, 2003; Puhl \& Heuer, 2009; Thomas, Hyde, Karunaratne, Herbert, \& Komesaroff, 2008).

Theoretical accounts derived from addiction research suggest that an approach-avoidance conflict towards desired stimuli may result when consumption of desired stimuli is associated with positive reinforcement while simultaneously associations with negative consequences of consumption are activated (e.g. Breiner, Stritzke, \& Lang, 1999). The observed pattern of eye-movements initially towards food, and then subsequently away from food, might reflect similar evaluation mechanisms in overweight participants. Likewise, a study on chocolate craving observed a comparable approach-avoidance pattern in chocolate cravers, suggesting that this ambivalence may occur in response to "forbidden" but desired stimuli (Cartwright \& Stritzke, 2008).

Further, these results might suggest that attention direction on desired stimuli represents the automatic attraction of these cues, whereas attentional avoidance of desired stimuli might be a voluntary strategy to resist consumption. In line with this view, a similar attention allocation process has been observed in studies with alcohol-dependent patients: Several studies showed that alcoholics who are trying to remain abstinent, for example clinic attendants, showed an approach-avoidance pattern towards alcohol stimuli, whereas social (heavy) drinkers and alcohol dependent patients with a short duration of abstinence did not (Noël et al., 2006; Townshend \& Duka, 2007; Vollstädt-Klein, Loeber, von der Goltz, Mann, \& Kiefer, 2009).

Further support for this idea comes from research on attention allocation to food in restrained eaters (individuals with chronic weight concerns who have the intention to lose weight), showing a similar pattern of approach and avoidance when confronted with food images (Hollitt, Kemps, Tiggemann, Smeets, \& Mills; Veenstra, de Jong, Koster, \& Roefs). Similarly, Nijs et al. (2010) suggested that obese participants in their study might have used cognitive techniques to reduce attention allocation on food stimuli, possibly in an attempt to prevent excessive food intake. Thus, there seem to be two feasible explanations accounting for the observed approach-avoidance pattern of attention allocation: overweight participants might have more ambivalent feelings towards high-fat food than normal weight participants and this becomes apparent in their visual attention patterns. Or, overweight participants might try to avoid high-fat food stimuli in order to prevent feeling tempted by the sight of it.

A further aim of this study was to investigate the relation between attentional biases and craving. Results yielded uniquely for overweight participants that selfreported craving prior to the visual probe task was associated with a stronger bias in initial orientation towards foods. This finding stresses specifically for over- 
weight participants the relevance of craving with regard to initial attention allocation. It highlights the dilemma of overweight people when exposed to highly palatable food cues: if craving enhances attentional biases for these food cues, this may increase the likelihood of their noticing any minor cues relating to highly palatable food in their environment (potentially triggering food-related intrusive thoughts and preoccupations), which might in turn further increase the temptation to indulge in these foods (though this latter consequence was not supported by the current data), and attentional avoidance of food stimuli might serve as a strategy to escape this vicious circle.

The results of the current study may provide further insight into the relation between attentional biases and food consumption: Overweight participants ate significantly more snack food than did healthy-weight participants during the bogus taste test. Note that this difference cannot be explained by differences in hunger or craving. This result might suggest that despite a quick attention shift away from food, overweight participants found it hard to control their eating behaviour when exposed to palatable high-calorie foods. This finding might further suggest that the effect of attentional avoidance might be short lived: Apparently, quickly looking away from food did not help overweight participants to resist palatable food that was offered to them 15 minutes later (during the bogus taste test).

The results of this study should be interpreted under the consideration of some limitations: The causal interplay of our main variables cannot be deduced based on our results. Future research should examine the causal relations of craving, attention allocation processes and eating behaviour experimentally, for example by inducing craving or attention biases. Considering that our sample consisted of young female students, it is questionable whether the results can be generalized to the wider population. It is to note that we conducted this study within a relatively small sample; nevertheless, we were able to show significant differences between groups in initial attentional biases. Moreover, our sample, who consisted of mainly mildly overweight individuals, did not show the expected attention maintenance component in gaze dwell time (i.e. increased dwell time on food cues assessed across the whole duration of stimulus presentation) that was earlier found in heavy obese participants (Castellanos, et al., 2009). Future studies should further investigate the possible differences in attention allocation processes between overweight and heavy obese participants. This difference between overweight and heavy obese participants might indicate that specific attention components might be involved at different stages in the process of developing obesity. Future research may further explore this development, for example by testing whether specific attention biases are related to weight changes in the future.

With these specifications in mind we draw the following conclusions: The results emphasize the environmental influence on information processing of food cues, that is attention allocation, and subsequent food intake, at least for over- 
weight individuals. Extensive advertising and aggressive marketing of high-fat food products, designed to catch attention, might have a differential impact on healthy-weight versus overweight individuals. The results of the current study point out that it might be problematic for overweight individuals to resist these tempting offers, because they might be more likely to spot high-fat foods, particularly while craving, and might then have difficulties to refrain from eating high-fat foods, contributing to further weight gain. A general assumption in obesity research is that the so called "toxic" food environment plays a crucial role in the dramatic increase of overweight and obesity rates in the western world. Individuals who are selectively paying attention to high-calorie food cues in this environment might be at increased risk to develop obesity. However, few studies have directly tested whether attentional biases for the high-calorie food items indeed play a role in this development. To our best knowledge the current study is the first to identify, in overweight/obese individuals, a distinctive pattern of cognitive bias in specific components of attention, which were directly assessed through eye movement recording: i.e., enhanced initial orientation to palatable food cues, which was positively related to food-craving, but which was rapidly and immediately followed by reduced attentional maintenance on those food cues.

In summary, the current study provides evidence for individual differences in attention biases for high-fat foods in overweight versus healthy-weight participants. Overweight participants showed an approach-avoidance pattern of attention allocation towards high-fat food: overweight participants directed their first gaze more often towards the food picture but did not maintain their initial gaze on this picture. Furthermore, overweight individuals consumed more food on a subsequent taste test, and correlational data indicated a unique association between craving and attention bias in initial orientation to food cues for overweight participants. These results highlight the importance of clarifying the role of attentional mechanisms contributing to overeating in overweight individuals. 



\section{Chapter 3}

\section{The power of food:}

\section{Attention bias for high-calorie food predicts weight gain in obese children}

Submitted as:

Werthmann, J. Roefs, A., Nederkoorn, C., Schyns, G., \& Jansen, A. (2013).

The power of food: Attention bias for high-calorie food predicts weight-gain in obese children. Manuscript in revision. 


\section{Abstract}

Objective: Obesity prevalence among children is increasing rapidly and knowledge on cognitive factors that contribute to children's reactivity to the obesogenic food environment could help to design effective treatment and prevention campaigns. Empirical studies in overweight and obese adults suggest that attention for food could be a risk factor for overeating.

Method: Accordingly, the first aim of the current study was to examine whether obese children show an attention bias to food whereas healthy children do not. The second aim was to test whether attention biases for food in obese children predict weight change over a period of three and six months. First, attentional biases for food were tested in obese children $(n=30)$ and matched healthy weight children $(n=30)$ by recordings of response latencies and eye-movements during a visual probe task with food and neutral stimuli. Second, a regression analysis was conducted to study if food-related attentional biases in obese children $(n=32)$ predicted weight changes after three and six months.

Results: Results showed that obese children did not differ in their attention to food from healthy-weight children. Yet, automatic attention for food (i.e., initial orientation bias) significantly predicted obese children's weight change after six months ( $\left.p=.032, R^{2}=.14\right)$.

Conclusions: Apparently, high palatable food is a salient stimulus for all children, irrespective of their weight status. However, automatically directing attention to food cues might facilitate further weight gain in obese children.

Keywords: Obesity, Childhood, Attention bias, Weight gain, Eye-tracking 
Obesity is a serious problem worldwide, for both adults and children. The prevalence of overweight and obesity among children is rising rapidly (Wang \& Lim, 2012). This development is extremely worrisome because childhood obesity is not only associated with several severe comorbid psychological and physical problems, but is also predictive of adult obesity and a risk factor for mortality (Cali \& Caprio, 2008; Pulgaron, 2013). Therefore, knowledge of factors that contribute to overeating, and thereby to the development of obesity, is imminent for effective treatments and prevention strategies.

The western world can be considered a so-called obesogenic environment: High-calorie and palatable foods are available everywhere and marketed aggressively, particularly to children (Halford, Gillespie, Brown, Pontin, \& Dovey, 2004; Harris, Bargh, \& Brownell, 2009; Jordan, Kramer-Golinkoff, \& Strasburger, 2008; Kelly et al., 2010). Yet, children might differ in their susceptibility to these constant food temptations. That is, all western children live in the same obesogenic environment, but many of them are not obese. In this respect it is important to study (cognitive) mechanisms, which could cause some children to be overly responsive to these temptations.

Biased attention to palatable, high-calorie food could be one possible vulnerability factor that contributes to overeating (e.g., Berridge, 1996; Castellanos et al., 2009; Werthmann et al., 2011). Research on attention for food and its relation to body weight has mainly been conducted in adults, and findings are mixed. For example, one study found that severely obese participants directed their first gaze more often towards food (i.e., initial orientation bias) and maintained their attention longer on food cues than healthy weight participants, when fed (Castellanos, et al., 2009). However, another study, examining attention in overweight versus healthy weight participants, found an attentional approach-avoidance reaction in overweight participants: they directed their attention more often towards food stimuli (i.e. initial orientation), but the fixation duration of their first gaze was significantly shorter (i.e., avoidance) in comparison to healthy-weight participants (Werthmann, et al., 2011). Another study that examined attention bias for food cues measured event-related potentials (ERP), and found an automatic or early attention bias (indicated by larger P200 waves based on recordings of electroencephalographic (EEG) brain activity) for food words in obese compared to healthyweight participants (Nijs, Franken, \& Muris, 2010). Similarly, another study also found that particularly obese participants tended to have a stronger automatic attentional bias for food cues, when response latencies during a visual probe task were assessed (Nijs, Muris, Euser, \& Franken, 2010). In contrast, other research found a negative correlation of initial orientation towards food and body-mass index (BMI) in a sample with mainly healthy weight participants (Nummenmaa, Hietanen, Calvo, \& Hyona, 2011). However, when testing cognitive correlates of BMI in a student sample, researchers found no association of food-related atten- 
tional biases and BMI (Pothos, Tapper, \& Calitri, 2009). Yet, Calitri and his colleagues (2010) reported in a follow-up study on this healthy student sample that reaction times to unhealthy and healthy food words (as assessed by a Stroop task) were predictive of BMI change after one year. Thus, evidence remains contradictory because some findings suggest attentional approach (e.g., Castellanos, et al., 2009) whereas other report attentional approach-avoidance reactions (e.g., Werthmann, et al., 2011) or avoidance of food cues in individuals with overweight or obesity (e.g., Nummenmaa, et al., 2011). In addition, some studies even report no association of food-related attentional biases and BMI (e.g., Pothos, et al., 2009).

In comparison, there is a paucity of research on attentional processing of food cues in obese children. Only two studies have been conducted so far to examine information processing of food cues in children (Braet \& Crombez, 2003; Soetens \& Braet, 2007). In the first study, a food Stroop task was applied. Results showed that obese children were slower in naming the color of a colored food word than a neutral word, as compared to healthy weight children. Based on this finding it was concluded that obese children found it more difficult to suppress processing the meaning of food words and might thus be more pre-occupied with food, whereas healthy weight children were not distracted by the meaning of food words (Braet \& Crombez, 2003). In a second study (Soetens \& Braet, 2007), responses to an imbedded word task comprising high-calorie food words and matched control words in overweight and healthy weight adolescents (between 12 and 18 years) was studied as a measure for attentional processing of food cues. Participants were given six minutes to search a word-grid with non-words and words for highcalorie food words and neutral words and the total number of discovered food words served as measure for enhanced information processing of food cues. In this study no significant differences in the number of discovered food words were found between overweight and healthy weight adolescents with regard to the amount of discovered food words, indicating that the two groups did not differ in their processing of hidden food words (Soetens \& Braet, 2007). As with the findings of attentional bias in adults, these studies provide mixed evidence for disturbed information processing in obese or overweight children.

A possible explanation for the great diversity in research findings in both adult and pediatric samples could be the use of different methodologies: the above mentioned studies differed in their research populations (satiated or hungry, severely obese, overweight and healthy weight participants), the operationalization and assessment of attentional biases for food (e.g., food-Stroop task, visual probe task with assessment of manual response latencies or with the measurement of eye-movements, EEG based ERP assessment of attentional processes) and the target stimuli (healthy or unhealthy food words, palatable food pictures, high-calorie food pictures, sometimes shown alone, sometimes contrasted with stimuli). Both studies with pediatric samples relied on indirect tasks that do not inform on the 
underlying attentional processes and rely on the assessment of response latencies or sematic processing, which could be influenced by maturation processes (Wolters et al., 2012). Another recognized difficulty in interpreting results based on the indirect assessment of attention (for example the recording of response latencies) is that this assessment does not provide information on the direction of attentional processing and/or the underlying attentional components (e.g., Field, Munafó, \& Franken, 2009; Mogg, Bradley, Field, \& De Houwer, 2003).

In this respect, it is crucial for a better understanding of the role of visual attention for food in the development and maintenance of obesity in children to close this research gap and study food-related attentional allocation processes as closely and directly as possible. Direct measures of visual attention allocation inform more accurately on the attentional mechanism at work during food-cue processing and might also be less influenced by maturation processes. Therefore, we aimed to combine a standard measure for attention, the visual probe task, with the recordings of eye-movements next to the usual assessment of response latencies in the present study. Recordings of eye-movements during the visual probe task are dynamic and provide more direct indices of attention allocation processes than response latencies. Moreover, another advantage of tracking of eye-movements is that this method can easily distinguish between such components within each trial. As results from food-related attention research in adults demonstrated, it is important to distinguish between different attentional components: initial attention orientation and initial fixation durations present more automatic aspects of attentional bias and may accordingly reflect other motivational, more spontaneous, processes than attention components that represent attention maintenance. Total dwell time on food stimuli or recordings on response latencies when using longer stimulus presentation durations reflect presumably more controlled and willful attention components that might thus be related to more conscious goal or motivation processes (Castellanos, et al., 2009; Field, Mogg, Zetteler, \& Bradley, 2004; Field, et al., 2009; Werthmann, et al., 2011). Thus, the concurrent eye-tracking during a visual probe task provides a more precise measure that can inform on different attentional components.

The aim of the current study was thus to study attentional biases for food and extend previous findings by applying a dynamic and direct measure of visual attention to test if obese children and adolescents within an age-range of six to 18 years would show a distinct pattern of attentional bias for high-calorie food when compared to matched healthy weight children and adolescents. Another aim was to extend the cross-sectional design, and to test prospectively whether attentional bias could predict weight change over three and six months in the obese sample.

In sum, we expected that obese children would show enhanced attentional biases towards high-calorie food images in comparison to healthy weight children (hypothesis 1). In addition, we hypothesized that stronger attentional biases for 
food would be predictive of reduced weight loss or increased weight gain at 3 and 6 months follow-up in obese children and adolescents (hypothesis 2).

\section{Method}

\section{Participants}

34 children with obesity who were enrolled in an outpatient pediatric obesity lifestyle intervention at the local academic hospital and 36 healthy weight children recruited from local schools were tested. All participants received the same information about the study with an information letter and an informed consent form. Recruitment procedures differed slightly per participant group. Children with obesity and their parents were approached in the pediatric obesity unit at the hospital, and were asked to participate in the current study at one of their first appointments there, prior to the start of a lifestyle intervention for weight loss. The children with obesity took part in the current study during the first six months of the lifestyle intervention, which was planned to continue for a longer duration. Moreover, the children with obesity included in our study represented only a small subsample of the children who will be included in the lifestyle intervention. Clinical effectiveness results regarding the lifestyle intervention, as well as a description of the lifestyle intervention, will be reported in due time in a separate report.

Healthy-weight children were approached in their schools. Here, children received information verbally by one of the experimenters and their parents received information leaflets. Interested participants were informed that they first had to indicate their weight and height and would be matched on gender and age to participating children with obesity. Thus, healthy weight children were informed that they were only eligible for participation if they had a healthy weight (based on self-report) and would match in the mean age and gender range of included children with obesity. If parents and their children provided informed consent they were invited for participation.

Six school children were tested but not included in analyses, because at the end of data collection, they did not match tested obese participants in age and/or gender $(n=4)$ or because assessment of BMI at the end of testing indicated that they were overweight $(n=2)$. So, 30 children with obesity and 30 healthy-weight children who were matched individually on age and gender were included in data analyses for testing hypothesis 1 (i.e., differences in attentional biases between children with obesity and healthy weight children). Matching was successful, in that the groups of children with obesity and healthy weight children did not differ on age, $t(29)=0.14, p=.89$, or gender distribution, $\chi^{2}(1,60)=0.00, p=1$, see Table 1 for characteristics. The percentage of overweight was calculated based on the 
body mass index (BMI) divided by the national (Dutch) norm BMI (Van Winckel \& Van Mil, 2001), adjusted for gender and age, $\times 100$ (for a similar classification see e.g., Braet \& Crombez, 2003; Nederkoorn, Coelho, Guerrieri, Houben, \& Jansen, 2012; Soetens \& Braet, 2007). According to this percentage a value of $100 \%$ indicates $0 \%$ overweight. A BMI percentage of $90-120 \%$ is regarded as a healthy BMI range for a child. A percentage of $120 \%-140 \%$ of the ideal weight is classified as overweight, a percentage of $140-160 \%$ is classified as moderate obesity, and a percentage of $160 \%$ is regarded as serious obesity. According to this classification, $9(30 \%)$ of the tested children with obesity were moderately obese and 21 (70\%) were severely obese. All healthy weight children had an adjusted BMI within the healthy weight range. For mean adjusted BMI per group, see Table 1.

Three and six months follow-up data on BMI for 31 and 32 children with obesity, respectively, was available through the assessment in the hospital and was used to test hypothesis 2 (i.e., whether attentional biases for food cues predict weight change over time).

\section{Pictorial visual probe paradigm}

Overview. Attention allocation towards high-calorie food stimuli was measured during a visual probe task with concurrent recordings of eye movements as a direct measure of attention allocation, and the assessment of response latencies as an indirect index of attention allocation. In the visual probe task, two images are presented simultaneously side by side followed by a probe $(*)$ appearing in the location of one of the images. Children were instructed to indicate the location of the probe as quickly as possible by pressing a corresponding key on a button box. Children first completed a brief practice round to get familiarized with the visual probe task.

Timing Trials. Each trial started with a central fixation cross, which remained on the screen for $100 \mathrm{~ms}$. Subsequently, the target image pair was presented for $3000 \mathrm{~ms}$. Then the probe, $\left({ }^{*}\right)$, was presented until the participant responded by pressing the appropriate key on a button box.

Trial types. A brief practice round with 10 trials was included prior to the actual task. The actual visual probe paradigm included 80 trials in total: 64 critical trials and 16 filler trials. These trials were divided into 2 blocks of each 40 trials. Critical trials consisted of 16 stimulus pairs, which each was presented four times. Filler trials consisted of 4 stimulus pairs, each was presented four times. The position of the probe was equally distributed per stimulus type and equally often on the left and right side of the screen. The order of trials was randomized uniquely for each participant. 
Stimuli. In critical trials, the image pair consisted of a picture of a high-calorie food item and a picture of an animal, for example vanilla muffins and little ducklings. Filler trials consisted of picture pairs depicting two neutral non-food photographs (shoes and furniture). All image pairs were matched as closely as possible with regard to colour, complexity and brightness, and size of the depicted object. To account for differences in attractiveness between food and animal stimuli that may account for differences in attentional biases, a pilot study was conducted. First, pictures of animals were matched with regard to visual features with pictures of palatable, high-calorie food for critical pictures pairs and pictures of furniture and shoes were matched on visible feature to create neutral picture pairs. In the pilot, a pool of 48 matched critical picture pairs and 18 matched neutral picture pairs were judged on valence and attractiveness of both depicted objects by 65 school children. Based on these rating, the 20 picture critical pairs in which the food stimuli and the animal stimulus were rated most attractive and at the same time most similar in terms of attractiveness were used in this study (Mean ratings animals $=3.89, \mathrm{SD}=0.5$; Mean ratings food $=4.07, \mathrm{SD}=0.4$, overall Mean ratings critical pairs $=3.88, \mathrm{SD}=0.3$ ) For filler trials 10 of the most neutrally and similarly rated filler pairs were included (Mean ratings neutral pairs $=2.99, \mathrm{SD}=0.3$ ). Each picture was presented equally often on the left and on the right side of the screen. For practice trials, pictures pairs consisted of photographs of non-food items, which were different from the photographs used for filler trials.

Eye movement measurements. Eye movements were recorded by a desktop mounted EyeLink 1000 system (SR Research Ltd., Mississauga, Ontario, Canada). A 9-point calibration with subsequent validation procedure was conducted prior to the visual probe paradigm. To assess attention allocation processes, participants' gaze fixations were studied. Gaze fixations were defined as any period that is not a blink or saccade and lasts at least $100 \mathrm{~ms}$ (Eyelink Dataviewer User's Manual, 2002-2008, SR Research Ltd.). Eye movements that occurred before the presentation of an image pair were excluded, because these movements could represent anticipatory fixations.

For analysis purposes, the computer screen was, invisible for participants, divided into three areas of interest: the mid-section, which indicated the location of the fixation cross, and the left and right section, representing the locations of the picture stimuli. Only eye movements in critical trials directed either to the left or the right section of the screen were extracted for further analyses. Eye movements in filler trials and gaze fixations in the mid area were excluded from further analyses. Eye movements were extracted using Data Viewer (SR Research Ltd., Mississauga, Ontario, Canada).

Attention Bias Scores. Three attention bias scores were derived from the eye movement data: initial orientation bias scores, initial gaze duration bias scores and gaze dwell time bias scores. 
An initial orientation bias reflects an early attentional process and is calculated based on the number of first fixations that are directed to a high-calorie food picture as a proportion of all trials on which a first fixation is made to either picture. A bias score of $50 \%$ indicates no bias for food, a bias score greater than $50 \%$ represents a higher proportion of first fixations directed to high-calorie food stimuli, whereas a bias score less than $50 \%$ indicates a higher proportion of first fixations directed to neutral stimuli.

The initial gaze duration bias is seen as a measure for early attention maintenance (e.g. Bradley, Mogg, Wright, \& Field, 2003). This bias is calculated based on the sum of multiple fixations occurring within the region of the initially fixated picture before gaze is shifted away. This means that the initial fixation may be followed by a second or third fixation within the same picture. The sum of these fixations indicates initial gaze duration before the person looked away from the picture. Initial gaze durations per image category (high-calorie food or neutral) were averaged over the relevant trials per participant. Bias scores for the initial gaze duration were computed by subtracting the mean duration of initial fixation directed to neutral images from the mean duration of initial fixation directed to highcalorie food images. Thus, a positive score is indicative of longer initial attention maintenance on high-calorie food stimuli, whereas a negative score is indicative of the reverse: longer initial maintenance on neutral stimuli.

Gaze dwell time is informative regarding the maintenance of attention on critical stimuli (e.g. Mogg, Field, \& Bradley, 2005). Overall dwell time per image category (high-calorie food vs. neutral) was summed in each critical trial, and then averaged per image category over all trials, resulting in an average total dwell time per image category. For the gaze dwell time bias score, the mean dwell time on neutral images was subtracted from the mean dwell time on high-calorie food images. Thus, a positive score indicates that attention was maintained longer on highcalorie food items than on neutral items, whereas a negative score indicates the reverse: longer maintained attention on neutral items.

Manual response latencies to probes. The logic of the visual probe task presumes that participants are faster to respond to probes appearing in the location of the stimulus that they attended. Conversely, it is assumed that participants are slower to respond to probes appearing in the location of the stimulus that they did not attend (MacLeod, Mathews, \& Tata, 1986). Participant's manual response latencies when indicating the location of the probe were used to calculate response latency bias scores. Based on a stimulus duration of $3000 \mathrm{~ms}$ the response latency bias indirectly reflects a maintained attention process. Response latencies were excluded from further analyses if they were faster than $200 \mathrm{~ms}$, slower than $2000 \mathrm{~ms}$, and then if they deviated more than 3 SDs from each participant's mean (e.g. Castellanos, et al., 2009; Mogg, Bradley, Hyare, \& Lee, 1998). Response latency bias scores were then calculated by subtracting the mean response latency on con- 
gruent trials (that is, when the probe replaced a high-calorie food image) from the mean response latency on incongruent trials (that is, when the probe appeared in the same location as the preceding neutral image). A positive bias score indicates an attention bias towards high-calorie food, whereas a negative bias score indicates an attention bias away from high-calorie food.

\section{Procedure}

All children were tested individually in a dimly lit room between 9 am and 12 am. Healthy weight children were tested at their respective schools and children with obesity were tested in a laboratory room at the Faculty of Psychology \& Neuroscience, as part of a general baseline assessment of their physical and psychological condition at the hospital. After a brief validation procedure to adjust eye-tracking parameters, children first completed the ten practice trials and subsequently continued with the first half of the visual probe task. After a short break, children completed the second half of the visual probe task. Then children or their parents provided information on age and school type. Finally, weight and height were measured to calculate the BMI and the adjusted BMI. Weight and height for children with obesity was subsequently assessed at their 3 month and their six month follow-up appointment at the hospital. All children received compensation with the value of $€ 7.50$ for their participation in the experiment. The current study received ethical approval from the ethics committee of the Faculty of Psychology \& Neuroscience at Maastricht University and from the medical-ethical committee at the local academic hospital.

\section{Results}

Do attentional biases differ between healthy weight children and children with obesity?

To test the first hypothesis, healthy weight children and children with obesity were compared on attentional bias measures (initial direction, initial fixation duration, dwell time, response latencies) by means of paired $t$-tests. Allocation patterns for food did not differ on any of the attentional bias measures between children with obesity and healthy weight children, all $t s<1.50$, all $p s>.14$, see Table 1 for exact statistics. Further, one sample $t$-tests were conducted for the whole sample to test if children in general directed more attention to food cues than to neutral cues. A one-sample $t$-test for initial orientation bias (tested against 50 , indicating no bias) indicated that all children directed their first gaze more often towards food cues than neutral cues $(M=53.21, S D=8.58), t(59)=2.90, p=.005$. Moreover, all chil- 
dren also maintained their first gaze longer on food cues than on neutral cues $(M=$ $73.95, S D=270.96$ ), as indicated by a one sample $t$-test against 0 (indicating no bias) for initial duration bias scores, $t(59)=2.11, p=.039$. Further one-sample $t$ tests against 0 (indicating no bias) yielded no significant results for dwell time bias $(M=56.30, S D=350.14), t(59)=1.24, p=.22$, and response latency bias $(M=-$ $8.51, S D=59.04), t(59)=1.12, p=.27$, indicating that attention was not maintained longer on food images than neutral images in later attention processes in the whole sample.

Table 1.

Participants characteristics and mean attentional bias scores statistical tests of group differences between healthy weight and obese children.

\begin{tabular}{|c|c|c|c|c|c|}
\hline & \multicolumn{2}{|c|}{$\begin{array}{l}\text { Healthy-weight } \\
\text { children } \\
(n=30)\end{array}$} & \multicolumn{2}{|c|}{$\begin{array}{l}\text { Obese children } \\
(n=30)\end{array}$} & \multirow[b]{2}{*}{ Statistics } \\
\hline & $M$ & $S D$ & $M$ & $S D$ & \\
\hline Age & 11.82 & 2.99 & 11.91 & 2.93 & $t(59)=0.12, p=0.91$ \\
\hline Gender & 13 boys & 7 girls & 13 boys & girls & $\begin{array}{l}\text { Pearson Chi }- \text { Square }=0.0, p= \\
1.00\end{array}$ \\
\hline BMI & 17.16 & 1.85 & 30.61 & 4.81 & $t(59)=14.28, p<0.001$ \\
\hline Adjusted BMI a & 97.49 & 8.95 & 176.05 & 23.05 & $t(59)=17.40, p<0.001$ \\
\hline School type & $\begin{array}{l}15 \text { prim } \\
\text { seconda }\end{array}$ & $\mathrm{y} / 15$ & $\begin{array}{l}19 \text { prim } \\
\text { seconda }\end{array}$ & $/ 11$ & $\begin{array}{l}\text { Pearson Chi }- \text { Square }=1.83, p= \\
0.40\end{array}$ \\
\hline $\begin{array}{l}\text { Initial orientation } \\
\text { bias } \underline{\text { a }}\end{array}$ & 53.51 & 9.85 & 52.90 & 7.23 & $t(59)=0.27, p=0.79$ \\
\hline $\begin{array}{l}\text { Initial gaze duration } \\
\text { bias }^{\text {b }}\end{array}$ & 94.27 & 271.66 & 53.65 & 273.35 & $t(59)=0.58, p=0.56$ \\
\hline Dwell time biasb & 123.27 & 338.46 & -10.68 & 354.38 & $t(59)=1.50, p=0.14$ \\
\hline $\begin{array}{l}\text { Response latency } \\
\text { bias }^{\text {b }}\end{array}$ & -5.34 & 47.24 & -11.69 & 69.56 & $t(51.1)^{c}=0.41, p=0.68$ \\
\hline
\end{tabular}

Note. RS = Restraint Scale (Herman \& Polivy, 1980), BMI = Body Mass Index, adjusted BMI = body mass index (BMI) divided by the national (Dutch) norm BMI (Van Winckel \& Van Mil, 2001), adjusted for gender and age, $\times 100$. Initial orientation bias $=\mathrm{N}$ of first fixations on high-calorie food stimuli $/(\mathrm{N}$ of first fixations on high-calorie food stimuli $+\mathrm{N}$ of first fixations on non-food stimuli) $* 100$. Initial gaze duration bias = mean duration of the sum of initial fixations occurring within region of high-calorie food stimuli when initially fixated on - mean duration of the sum of initial fixations occurring within region of non-food stimuli when initially fixated on. Dwell time bias = mean total dwell time on high-calorie food stimuli - mean total dwell time on non-food stimuli. Response latency bias = Mean response latency in congruent trials - mean response latency in incongruent trials.

a in $\%$

$\mathrm{b}$ in $\mathrm{ms}$

${ }^{\mathrm{c}}$ unequal variances: $\mathrm{df}$ were corrected to 51.10 and $t$ and $p$ are reported accordingly. 
Do attentional biases predict change in BMI after three and six months?

Two multiple regression analyses were conducted to test if the attentional bias measures significantly predict BMI changes at three months or at six months, respectively (hypothesis 2). Follow-up data at three and six months were missing for two children who stopped coming to their regular appointments at the hospital, and for one child who missed the appointment at three months. Follow-up data on the remaining sample of 32 children with obesity was used for analyses at six months and for 31 children with obesity at three months. First, BMI change for three and six months was computed for all children with obesity. For BMI change after three months the adjusted BMI \% at time of testing was subtracted from adjusted BMI \% at three months. Similarly for BMI change after six months, the adjusted BMI \% at testing was subtracted from the adjusted BMI \% at six months. A positive score represents an increase in adjusted BMI \%, thus weight gain adjusted for gender and age, whereas a negative score represents a decrease in adjusted BMI \%, thus weight loss adjusted for gender and age. Mean adjusted BMI change was $-1.58 \%(\mathrm{SD}=8.88)$ at three months and $-2.40 \%(\mathrm{SD}=10.82)$ at six months. Inspection of a scatterplot suggested that one participant had an outlying decrease in adjusted BMI scores after three months (- $28.68 \%$ change in adjusted BMI, i.e., 3.05 SDs below the mean adjusted BMI change) and after six months (- $38.90 \%$ change in adjusted BMI, i.e., 3.30 SDs above the mean adjusted BMI change) and therefore these outlying BMI changes were recoded to the nearest BMI change score in the obese group (-19.58\%, i.e. 2.04 SDs below the mean adjusted BMI change, and $-19.21 \%$, i.e. 1.55 SDs below the mean adjusted BMI change, respectively) (see Wilcox, 2011) prior to the regression analysis ${ }^{1}$. For the regression analysis testing, all four attentional bias measures (initial orientation, initial fixation duration, dwell time and response latencies) were centred before being entered into the respective regression model. Results of the regression for BMI change at three months indicated that attentional bias measures did not significantly predict change in adjusted BMI after three months, see Table 2. Results of the regression for BMI change at six months showed that only initial orientation bias significantly predicted changes in BMI after six months, see Table 2 and Figure 1.

\footnotetext{
${ }^{1}$ Results remained the same when we included this participant with the original BMI change scores in our data analysis.
} 


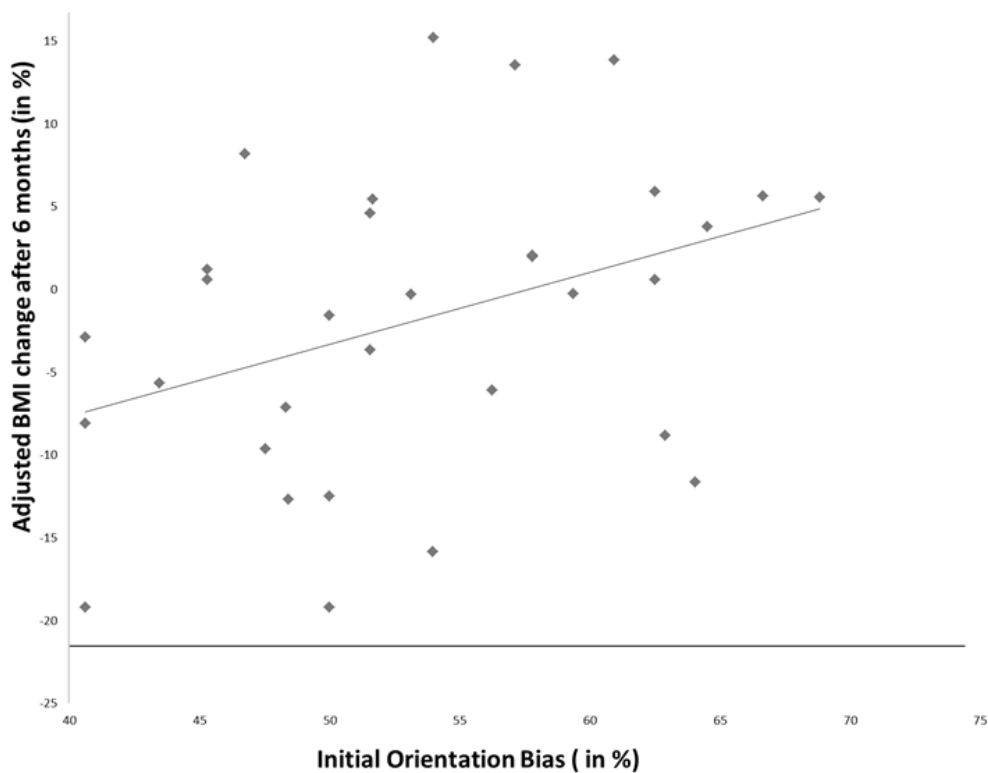

Figure 1. Correlation of adjusted BMI change after 6 months (in \%) in children with obesity ( $\mathrm{n}=32$ ) and initial orientation bias scores (in \%, a percentage above 50 indicates a bias towards food).

Table 2.

Regression coefficients of attentional bias scores predicting change in BMI after 3 and after 6 months in obese children: Multivariate regression analyses

\begin{tabular}{|c|c|c|c|c|c|c|c|c|c|c|c|c|c|c|c|c|}
\hline & \multicolumn{8}{|c|}{ BMI change at 3 months } & \multicolumn{8}{|c|}{ BMI change at 6 months } \\
\hline & \multicolumn{4}{|c|}{ Full Model } & \multicolumn{4}{|c|}{ Final Model } & \multicolumn{4}{|c|}{ Full Model } & \multicolumn{4}{|c|}{ Final Model } \\
\hline & $\mathrm{B}(\mathrm{SE})$ & $\beta$ & $\mathrm{t}$ & $p$ & $\mathrm{~B}(\mathrm{SE})$ & $\beta$ & $\mathrm{t}$ & $\mathrm{p}$ & $\mathrm{B}(\mathrm{SE})$ & $\beta$ & $\mathrm{t}$ & $p$ & $\mathrm{~B}(\mathrm{SE})$ & $\beta$ & $\mathrm{t}$ & $p$ \\
\hline $\begin{array}{l}\text { Initial } \\
\text { orientation }\end{array}$ & $\begin{array}{l}0.16 \\
(0.20)\end{array}$ & .16 & 0.77 & .45 & - & - & - & - & $\begin{array}{l}0.49 \\
(0.22)\end{array}$ & .43 & 2.28 & .03 & $\begin{array}{l}0.44 \\
(0.19)\end{array}$ & .38 & 2.24 & .032 \\
\hline $\begin{array}{l}\text { Initial } \\
\text { fixation } \\
\text { duration }\end{array}$ & $\begin{array}{l}<0.01 \\
(0.01)\end{array}$ & .04 & 0.14 & .89 & - & - & - & - & $\begin{array}{l}<0.01 \\
(0.01)\end{array}$ & -.13 & -0.58 & .57 & - & - & - & - \\
\hline Dwell time & $\begin{array}{l}<0.01 \\
(0.01)\end{array}$ & -.01 & 0.41 & .97 & - & - & - & - & $\begin{array}{l}<0.01 \\
(0.01)\end{array}$ & -.06 & -0.30 & .77 & - & - & - & - \\
\hline $\begin{array}{l}\text { Response } \\
\text { latencies }\end{array}$ & $\begin{array}{l}0.02 \\
(0.02)\end{array}$ & .14 & 0.71 & .47 & - & - & - & - & $\begin{array}{l}<0.01 \\
(0.02)\end{array}$ & $<.01$ & 0.04 & .97 & - & - & - & - \\
\hline Constant & $\begin{array}{l}-1.33 \\
(1.53)\end{array}$ & - & -0.87 & .39 & $\begin{array}{l}-1.29 \\
(1.45)\end{array}$ & - & -.89 & .38 & $\begin{array}{l}-1.91 \\
(1.58)\end{array}$ & - & -1.21 & .24 & $\begin{array}{l}-1.94 \\
(1.52)\end{array}$ & - & 1.28 & .21 \\
\hline
\end{tabular}

Note. To build the final model the least significant variable was dropped in a backwards regression analyses until only significant predictors remained. For BMI change at three months: $R=.24, R^{2}=0.06$, $p=.81$ for the full model; $\Delta \mathrm{R}^{2}=-.037$ for the final model, $R^{2}=.00, p=$ n.s. For BMI change at six months: $R=.41, R^{2}=.17, p=.27$ for the full model; $\Delta \mathrm{R}^{2}=-.022$ for the final model, $R^{2}=.14, p=.032$. 


\section{Discussion}

Attention biases for food might contribute to increased food intake and might therefore be a cognitive mechanism contributing to the development and/or maintenance of overweight and obesity. However, evidence for an attention bias in adults and children with obesity is mixed. The first aim of this study was to test whether children with obesity differ in their attention for food from healthyweight children. A second aim was to explore if attentional biases for food significantly predict subsequent weight change in children with obesity. To test these hypotheses, attention bias for food was measured in healthy weight children and in children with obesity by employing a visual probe task depicting food and animal stimuli with concurrent assessment of eye-movements and the measurement of response latencies. Our results provide no empirical evidence for differences in attentional biases for food cues between children with obesity and healthy weight children. Instead, our results suggest that all children have a tendency to orientate towards tasty high-calorie food cues (i.e. initial orientation bias) and initially maintain their first gaze longer on food cues than on neutral cues (i.e. initial fixation duration bias). These findings suggest that tasty high-calorie food cues are highly salient stimuli for all children. Considering that food stimuli were paired with almost equally liked animal pictures in our attention paradigm, our results stress the "power of food" on attentional processing in children by indicating that even if another attractive object is available, food items are more powerful in catching children's attention first.

While the observation that all children, not only children with obesity, have an attention bias for food cues is in line with some similar findings in adults that also indicated that everyone has an attentional bias for food (e.g., Nijs, Franken, \& Muris, 2008; Werthmann, Roefs, Nederkoorn, \& Jansen, 2013; Werthmann et al., 2013), they are not in line with studies that demonstrated differences in attention bias for food in obese and healthy weight adults and children (Braet \& Crombez, 2003; Castellanos, et al., 2009). There are two possible explanations for this diversity of results, related to the choice of paradigm and stimuli. Different paradigms tap into different attentional processes and indirect measures of biased attention might reflect other processes than measurements of visual attention. Moreover, the stimulus selection might also be a possible influence on attentional processes. Previous research that measured food-related attention often contrasted (highcalorie) food items with a relatively neutral stimulus category, such as office supplies or musical instruments. In this respect our current study underscores the strength of an attentional bias for food cues by demonstrating that even in contrast to a highly liked contrast category, food cues are more potent in grabbing attention. Thus, the current study extends previous research on food-cue related atten- 
tional biases by showing that even in contrast to equally attractive other objects, food cues are more capable of grabbing early attention allocation.

Another explanation for the diverse results is that all children have an innate attention bias for food, yet attention for food might wane in healthy weight children during the course of their development, whereas attention for food might remain heightened, in contrast to other stimuli in the environment, in children with obesity. A similar process has been suggested for a comparable observation in the field of anxiety disorders: here research on attentional biases for threat cues in children found that all children had a threat-related attentional bias (Kindt, van den Hout, de Jong, \& Hoekzema, 2000). Accordingly, Kindt and van den Hout (2001) hypothesized that during childhood children learn to strategically inhibit an attentional bias towards threat, and anxious children may not acquire this skill adequately and therefore show persistence of attention bias to threat. Thus, selective attention to threat may decrease with age through increased encounters with threatening information and development of attentional control (Wolters, et al., 2012). Even though speculative, we suggest that a similar mechanism could underlie changes in attentional biases for food cues in children: those children who grow up paying less attention to food cues in comparison to other cues will have a healthy weight and be able to resist food temptations in the environment through the exercise of attentional control. In contrast, children who continue to have strong attentional biases for food cues in comparison to other cues might have a higher chance to overeat, gain weight and become obese.

In this respect, the results concerning our second hypothesis might be important. The current results showed that a bias in initial orientation towards food cues positively predicted weight gain after six months in children with obesity. This could be another piece of evidence for the (hypothesized) role of attention in the etiology and maintenance of obesity: directing attention towards food might be a vulnerability factor for overconsumption, and thus subsequent weight gain. However, based on our results it is not clear if this process is specific for children with obesity or could also be observed in healthy weight children. Future research should examine if a similar association of food-related initial orientation and BMI change exists in healthy weight children. A previous study indicated that the Stroop interference effect for unhealthy food words indicated weight gain in a mainly healthy weight student sample, whereas Stroop interference scores for healthy food words negatively predicted weight change (Calitri, et al., 2010). Thus, it would also be interesting to examine whether attentional biases for healthy food could influence later BMI. This knowledge could be important for the design of effective weight-loss interventions in children and adults.

In addition, it is interesting to note that weight gain was associated with an early attention component, because previous research on substance use and food intake suggested that later attentional components, namely maintained attention, 


\section{CHAPTER 3}

was associated with subsequent consumption (Werthmann, Roefs, Nederkoorn, \& Jansen, 2013). A possible explanation for this divergence could be that later attentional components reflect a conscious decision about consumption, and thereby predict subsequent consumption. Early attentional processes on the other hand might reflect less conscious motivational vulnerability to overconsumption, and thereby predict weight trajectories.

Taken together, the current findings might partly explain how the current food environment directs children's eating behavior; food cues grab the attention of all children, even when other attractive alternatives are available. Our results moreover suggest that biased looking at high-calorie foods increases the chance of future weight gain in children with obesity. Our results stress the "toxic" impact of an obesogenic food environment on children's perception and on obesity in children. 


\section{Chapter 4}

\section{Attention bias for food is independent of restraint in healthy weight individuals: \\ An eye tracking study}

Published as:

Werthmann, J., Roefs, A., Nederkoorn, C., Mogg, K., Bradley, B. P., \& Jansen, A. (2013). Attention bias for food is independent of restraint in healthy weight individuals-An eye tracking study. Eating Behaviors, 14(3), 397-400. 


\section{Abstract}

Objective: Restrained eating style and weight status are highly correlated. Though both have been associated with an attentional bias for food cues, in prior research restraint and BMI were often confounded. The aim of the present study was to determine the existence and nature of an attention bias for food cues in healthy-weight female restrained and unrestrained eaters, when matching the two groups on BMI.

Method: Attention biases for food cues were measured by recordings of eye movements during a visual probe task with pictorial food versus non-food stimuli. Healthy weight high restrained $(n=24)$ and low restrained eaters $(n=21)$ were matched on BMI in an attempt to unconfound the effects of restraint and weight on attention allocation patterns.

Results: All participants showed elevated attention biases for food stimuli in comparison to neutral stimuli, independent of restraint status.

Conclusions: These findings suggest that attention biases for food-related cues are common for healthy weight women and show that restrained eating (per se) is not related to biased processing of food stimuli, at least not in healthy weight participants.

Keywords: Attention, cognitive bias, eye movements, food cues, dietary restraint, BMI 
Attention biases for food have been associated not only with craving, (over)consumption and overweight or obesity (e.g., Nijs, Muris, Euser, \& Franken, 2010; Smeets, Roefs, \& Jansen, 2009; Werthmann et al., 2011) but also with restrained eating (e.g., Hollitt, Kemps, Tiggemann, Smeets, \& Mills, 2010; Veenstra, de Jong, Koster, \& Roefs, 2010). Overweight and obese people typically report higher levels of restraint than healthy-weight people (Johnson, Pratt, \& Wardle, 2011). Therefore, the aim of this study was to test whether restrained eating style, independently of body weight, is associated with an attentional bias for food cues.

Evidence of an attention bias for food in restrained eaters is mixed. For example, minor effects for biased attention for food words in restrained eaters, as assessed by the modified Stroop-paradigm, were reported in meta-analyses (e.g., Brooks, Prince, Stahl, Campbell, \& Treasure, 2011). Yet, the interference effect does not inform about the underlying visual attention processes (Mogg, Bradley, Field, \& De Houwer, 2003). Other studies have used paradigms that can distinguish specific attention processes (i.e., visual search, visual probe and flanker paradigm). Some found that restrained eaters have an attention bias towards high-calorie food cues (Hollitt, et al., 2010; Meule, Vögele, \& Kübler, 2012), or avoided attending highcalorie food pictures in an exogenous cueing task (Veenstra, et al., 2010), or showed no conclusive evidence of an attention bias for food (Ahern, Field, Yokum, Bohon, \& Stice, 2010; Boon, Vogelzang, \& Jansen, 2000; Forestell, Lau, Gyurovski, Dickter, \& Haque, 2012; study 1).

Apart from the large variety of paradigms that have been used to assess attentional bias for high-calorie foods in restrained eaters, an important problem is that typically body weight (as indicated by the body mass index; BMI) and restraint status have been confounded in prior research. Attention biases for food might be primarily associated with weight problems, and might therefore be more related to weight than restraint per se. Thus, there are two competing explanations for the existing evidence of an attentional bias for food in restrained eaters: Restrained eating itself causes the attentional bias, or, alternatively, heightened BMI, typically associated with restraint, is the cause of the attentional bias.

Our first aim was to test whether restrained eating is related to increased attention bias for food, independent of weight status. We isolated the influence of restraint methodologically by keeping BMI constant (within a healthy BMI range) and varying the distribution of restrained eating behaviour. Thus, our study contributes to unconfounding the effects of BMI and restraint on attentional bias for food.

Apart from the question of whether restrained eating style, independently of BMI, affects attentional processing of food cues, a second aim of this study was to explore the direction and duration of the potential attentional bias. Research on attention biases in eating disorders and addictions shows that these attention components are differently related to motivational processing of food (or drug) 
cues (Bradley, Mogg, Wright, \& Field, 2003; Field, Mogg, Zetteler, \& Bradley, 2004; Smeets, Roefs, van Furth, \& Jansen, 2008). It is possible that restrained eaters display attention avoidance of food cues, in an attempt to follow their dieting rules, or that restrained eaters show attentional approach, reflecting high preoccupation with food (Higgs, Rutters, Thomas, Naish, \& Humphreys, 2012; Lowe \& Levine, 2005). Another possibility is that restrained eaters show an approach-avoidance conflict (Papies, Stroebe, \& Aarts, 2008), for example, an initial orientation towards food (automatic approach), followed by diverting attention away from food in later stages of processing (strategic avoidance).

Therefore paradigms that can distinguish between these attentional components are more informative for the question of whether restrained eating is associated with attention avoidance of food cues (e.g. reflecting dieting motivation) or attentional approach towards food cues (e.g. reflecting food pre-occupation or eating enjoyment). In this respect, a meta-analysis recently concluded that eye movement recording is the most sensitive measure for attention biases in the context of addiction because it allows for an unambiguous distinction of specific components of attention allocation processes, and, due to the dynamic character, provides an assessment of change in attention biases over time following stimulus onset (Field, Munafó, \& Franken, 2009).

\section{Method}

\section{Participants}

Potential participants were screened on restraint and self-reported BMI $\left(\mathrm{kg} / \mathrm{m}^{2}\right)$ and matched as closely as possible on their self-reported BMI. Female healthyweight unrestrained $(n=21)$ and restrained eaters $(n=24)$, based on a mediansplit on their Restraint Scale scores (Herman \& Polivy, 1980) ( $M d n=11$; Range $=4$ 27), were included. Four additional participants were tested but excluded because their actual BMI did not meet the inclusion criterion of healthy weight (See Table 1).

\section{Pictorial visual probe paradigm}

Overview. Attention biases for high-calorie food were assessed from concurrent eye movements recording and manual response latencies during a visual probe paradigm with pictures of high-caloric food and non-food stimuli (for a complete description of this task, see Werthmann et al. (2011). The visual probe paradigm comprised 120 trials (80 critical and 40 filler trials) which were divided into two blocks of 60 trials. For critical trials, 20 picture pairs, with high-calorie food paired with non-food pictures (musical instruments), were presented four 
times. Picture pairs on filler trials showed neutral non-food objects, each presented four times. Practice trials also used pictures of common non-food items. Pictures used on filler and practice trials were different from those used for critical trials. Image pairs were the same as those used in Werthmann et al. (2011) and were presented for $2000 \mathrm{~ms}$. Each stimulus was presented equally often on the left and right side of the screen. The position of the probe was equally distributed per stimulus type and appeared equally often on the left and right side of the screen. The order of trials was randomized individually for each participant.

Eye movement measurements. Eye movements were recorded by a desktop mounted EyeLink 1000 system (SR Research, Mississauga, Ontario, Canada) and extracted using Data Viewer (SR Research, Mississauga, Ontario, Canada), exlcuding anticipatory eye movements, gaze fixations in the mid area and filler trials (see, Werthmann, et al., 2011). Three attention bias scores were calculated: (i) gaze direction bias: the proportion of trials on which the first fixation was directed to a food stimulus versus a non-food stimulus (a score above 50\% indicates a higher proportion of first fixations on food stimuli); (ii) initial gaze duration bias: a measure for early attention maintenance, defined as the difference between the sum of fixations between food and non-food stimuli, before gaze was shifted away from the initially fixated picture (a positive score indicates longer initial gaze on food, than non-food, stimuli), and (iii) gaze dwell time bias: the average total dwell time on food versus non-food stimuli (a positive score indicates a bias towards food stimuli), (e.g., Castellanos et al., 2009; Werthmann, et al., 2011).

Manual response latencies to probes. Response times (RTs) were excluded from analyses if they were faster than $200 \mathrm{~ms}$, slower than $2000 \mathrm{~ms}$, and then if they deviated more than $3 S D$ s from each participant's mean (Mogg, Bradley, Hyare, \& Lee, 1998). RT bias scores were computed by subtracting the mean RT on congruent trials (i.e., when the probe replaced a food image) from the mean RT on incongruent trials (i.e., when the probe replaced the neutral image). A positive bias score indicates an attention bias towards food.

\section{Questionnaires}

Restrained Eating. The Restraint Scale (RS; Herman \& Polivy, 1980) assesses weight concerns, weight fluctuations and self-reported attempts to diet, and was used to identify unrestrained and restrained eaters in this study.

Hunger. Subjective hunger was assessed by three $100 \mathrm{~mm}$ visual analogue scales (rating how hungry, how much craving, and how much she thought she could eat) and a compound measure was computed for data analyses. Additionally, the participant indicated the time passed since her last meal and time that would elapse until her next meal. 
Procedure

After informed consent and practice trials, the visual probe task was performed. Afterwards, the participant filled in the RS and hunger scales. Height (m) and weight (kg) were measured. Finally, the participant was debriefed and compensated. The local ethical committee approved the study.

\section{Results}

Descriptive statistics

Restrained and unrestrained eaters did not differ on hunger measures or BMI when they entered the lab (see Table 1), confirming that average BMI was matched between the groups.

\section{Attention bias scores}

Analyses group as a whole. One sample $t$-tests, comparing gaze direction bias scores against a score of 50\% (which indicates no attention bias), revealed a significant gaze direction bias for food cues in the whole sample $(M=52.28, S D=6.83$, $t(44)=2.24, p=.030)$. Overall, participants also showed a bias for food stimuli in later visual attention processes, as revealed by one-sample $t$-tests against 0 (indicating no bias), for initial gaze duration bias $(M=79.16, S D=121.71, t(44)=4.36, p$ $<.001)$; dwell time bias $(M=41.57, S D=127.42, t(44)=2.19, p=.034)$; and RT bias $(M=5.80, S D=18.03, t(44)=2.16, p=.037)$ scores.

Comparing restrained and unrestrained eaters. Restrained eaters did not differ from unrestrained eaters on any of the bias scores, as shown by independent $t$-tests (all $t^{\prime}$ s $<0.72$; all $p^{\prime}$ 's $>.47$ ), see Table 1 . 
Table 1.

Characteristics and attention bias scores of restrained and unrestrained eaters.

\begin{tabular}{|c|c|c|c|c|c|c|}
\hline & \multicolumn{2}{|c|}{$\begin{array}{l}\text { Restrained eaters } \\
(n=24)\end{array}$} & \multicolumn{2}{|c|}{$\begin{array}{l}\text { Unrestrained eaters } \\
(n=21)\end{array}$} & \multirow[b]{2}{*}{$t(43)$} & \multirow[b]{2}{*}{$p$} \\
\hline & $M$ & $S D$ & $M$ & $S D$ & & \\
\hline RS score & 15.75 & 4.34 & 7.19 & 1.97 & $8.70^{2}$ & $<0.001$ \\
\hline Age & 21.50 & 1.34 & 21.87 & 2.66 & 0.84 & 0.40 \\
\hline BMI & 21.77 & 1.59 & 21.11 & 1.60 & 1.38 & 0.17 \\
\hline Hunger & 47.95 & 20.49 & 47.36 & 28.10 & 0.08 & 0.94 \\
\hline Time since last meal (in min) & 107.67 & 63.80 & 139.29 & 88.15 & 1.39 & 0.17 \\
\hline Gaze direction bias $^{\mathrm{a}}$ & 51.97 & 6.56 & 52.63 & 7.26 & 0.37 & 0.75 \\
\hline Initial gaze duration bias ${ }^{b}$ & 66.81 & 123.59 & 93.28 & 120.94 & 0.72 & 0.47 \\
\hline Dwell time bias ${ }^{\mathrm{b}}$ & 32.71 & 128.03 & 51.68 & 129.10 & 0.49 & 0.62 \\
\hline Response latency bias ${ }^{b}$ & 5.99 & 18.87 & 5.57 & 17.48 & 0.08 & 0.94 \\
\hline
\end{tabular}

Note. RS = Restraint Scale (Herman \& Polivy, 1980), BMI = Body Mass Index. Gaze direction Bias = N of first fixations on high-calorie food stimuli / ( $\mathrm{N}$ of first fixations on high-calorie food stimuli $+\mathrm{N}$ of first fixations on non-food stimuli) $* 100$. Initial gaze duration bias = mean duration of the sum of initial fixations occurring within region of high-calorie food stimuli when initially fixated on - mean duration of the sum of initial fixations occurring within region of non-food stimuli when initially fixated on. Dwell time bias = mean total dwell time on high-calorie food stimuli - mean total dwell time on non-food stimuli. Response latency bias $=$ Mean response latency in congruent trials - mean response latency in incongruent trials.

${ }^{a}$ in $\%$

bin ms.

\section{Discussion}

The current study was conducted in an effort to unconfound the effects of restraint and weight-status on attentional bias for food and to examine the exact nature of the hypothesized attentional bias for food. Results indicated that, overall, participants showed increased attention to food stimuli relative to non-food stimuli on all attention bias scores. Moreover, these bias scores were not larger for restrained than unrestrained eaters.

These results suggest that attention biases for food cues are "normal" and apparent in healthy weight women, irrespective of restraint level. The lack of an

\footnotetext{
${ }^{2}$ unequal variances: $\mathrm{df}$ were corrected to 32,975 , and $t$ and $p$ were reported accordingly
} 
effect of restraint on attention bias could not be explained by other confounding factors, such as hunger or BMI, as these did not differ between the groups. These results are in line with previous studies that also reported no differences in attention biases for food between restrained and unrestrained eaters (Ahern, et al., 2010; Boon, et al., 2000; Forestell, et al., 2012: study 1). Former null results have often been the basis for speculations on whether null findings reflected a lack of attention bias or a zero net effect resulting from the co-occurrence of approach and avoidance processes. The present study did not support these latter speculations, while using an online assessment of eye movements, the most sensitive measure of visual attention currently available (Field, et al., 2009).

The present results, however, contrast with findings of previous studies that did report a significant difference in attention bias between restrained and unrestrained eaters (Hollitt, et al., 2010; Meule, et al., 2012; Overduin, Jansen, \& Louwerse, 1995; Veenstra, de Jong, Koster, \& Roefs, 2010). The present results suggest a possible reason for the divergence of previous results, namely that unconfounding of weight and restraint (as attempted in the current study) eliminates the formerly observed bias effect. Typically, restrained eating and BMI are highly correlated. Indeed, studies that reported an attention bias observed this bias in restrained eaters who were significantly heavier than unrestrained eaters (e.g. Meule, et al., 2012; Veenstra, et al., 2010). In combination with the present results, these findings suggest that not restraint per se, but weight problems (i.e., overweight and obesity) could have contributed to previously observed differences between restrained and unrestrained eaters. In line with this, several studies have reported differences in attention biases between healthy weight and overweight or obese participants (Castellanos, et al. 2009; Nijs, Muris, et al. 2010; Werthmann, et al. 2011).

In conclusion, restrained eating per se does not contribute to biased attentional processing of food stimuli, at least not within healthy weight females. Given that attention biases for food could be universally present, the relationship between attention biases and actual food intake should be further investigated. 


\section{Chapter 5}

Desire lies in the eyes:

Attention bias for chocolate is related to craving and self-endorsed eating permission

\section{Published as:}

Werthmann, J., Roefs, A., Nederkoorn, C., \& Jansen, A. (2013). Desire lies in the eyes: Attention bias for chocolate is related to craving and self-endorsed eating permission. Appetite, 70, 81-89. doi: 10.1016/j.appet.2013.06.087 


\section{Abstract}

Objective: The present study tested the impact of experimentally manipulated perceived availability of chocolate on attention for chocolate stimuli, momentary (state) craving for chocolate and consumption of chocolate in healthy weight female students. It was hypothesized that eating forbiddance would be related to attentional avoidance (thus diminished attention focus on food cues in an attempt to prevent oneself from processing food cues) and that eating motivation would be related to attentional approach (thus maintained attentional focus on food cues).

Method: High chronic chocolate cravers $(n=40)$ and low cravers $(n=40)$ participated in one of four perceived availability contexts (required to eat, forbidden to eat, individual choice to eat, and $50 \%$ chance to eat) following a brief chocolate exposure. Attention for chocolate was measured using eye-tracking; momentary craving from self-report; and the consumption of chocolate was assessed from direct observation.

Results: The perceived availability of chocolate did not significantly influence attention allocation for chocolate stimuli, momentary craving or chocolate intake. High chocolate cravers reported significantly higher momentary craving for chocolate $(d=1.29, p<.001)$, and showed longer initial duration of gaze on chocolate, than low cravers $(d=0.63, p<.01)$. In contrast, participants who indicated during the manipulation check that they would not have permitted themselves to eat chocolate, irrespective of the availability instruction they received, showed significantly less craving $(d=0.96, p<.01)$ and reduced total dwell time for chocolate stimuli than participants who permitted themselves to eat chocolate $(d=0.53, p<$ .05).

Conclusions: Thus, this study provides evidence that attention biases for food stimuli reflect inter-individual differences in eating motivation, - such as chronic chocolate craving, and self-endorsed eating permission.

Keywords: Attention, Eye-tracking, Craving, Eating Behaviour, Chocolate, Eating Motivation 
Attention biases for food cues have been studied extensively in the last decennia (see e.g., for a review Brooks, Prince, Stahl, Campbell, \& Treasure, 2011). An attention bias refers to selective information processing that favours personally salient/relevant information (e.g., food stimuli) over neutral information (MacLeod, Mathews, \& Tata, 1986). One unresolved issue is the extent to which attention bias for food cues reflects motivation for food (e.g., eating allowance, (over)eating, craving) and/or a current concern about food (e.g., eating forbiddance, cognitive restraint, trying to avoid food intake) (e.g., Brooks, et al., 2011; Dobson \& Dozois, 2004; Nijs, Franken, \& Muris, 2010). Therefore, the aim of this study is to clarify the role of eating motivation and eating forbiddance in determining cognitive, subjective and behavioural responses to desirable food, by studying the effect of chronic chocolate craving, and an experimentally controlled manipulation of food availability (i.e., being required to eat chocolate versus being forbidden to eat chocolate) on attentional bias for food, momentary craving, and food intake.

On the one hand, eating disorder patients (ED), that is, individuals who are concerned with eating and obsessed with weight loss (i.e., high "eating forbiddance"), showed elevated attention biases for (high-calorie) food stimuli in comparison to healthy controls (for a review see Brooks, et al., 2011). For example, a broad range of evidence on the food Stroop task shows an interference effect for food words in eating disorder patients when compared to healthy control participants (Dobson \& Dozois, 2004; Lee \& Shafran, 2004), however the direction of the attention process (attentional avoidance versus attentional approach) cannot be specified by means of the Stroop task. The direction of an attention bias is important, though, because knowing whether ED patients show an attention bias towards or away from food cues could have important theoretical and clinical implications.

However, also in studies using paradigms that are capable to distinguish attention components, evidence on the direction of this attention bias is mixed:. In one study ED-patients showed increased distraction specifically for high-calorie food words but not low-calorie food words during a visual search task, which was interpreted as attention bias towards food cues (i.e., attentional approach) (Smeets, Roefs, van Furth, \& Jansen, 2008). In contrast, two studies using pictorial food stimuli reported that ED patients, in comparison to healthy control participants or remitted ED patients expressed significantly negative attention bias scores for (positive) eating related pictures as measured by response latency recordings during a dot-probe task. Thus, ED patients diverted their attention away from food cues (i.e., attentional avoidance) (Shafran, Lee, Cooper, Palmer, \& Fairburn, 2007, 2008). Evidence from attention research in nonclinical populations suggests that dieting and restrained eating might also lead to elevated attention processes towards (or away from) food cues (Boon, Vogelzang, \& Jansen, 2000; Brooks, et al., 2011; Dobson \& Dozois, 2004). Restrained eaters are preoccupied 
with their food intake and their weight (Jones \& Rogers, 2003; Timmerman \& Gregg, 2003; Wardle, 1987). Their constant struggle to adhere to their dieting rules could be reflected in biased processing of (high-calorie) food cues (Higgs, Rutters, Thomas, Naish, \& Humphreys, 2012; Tiggemann \& Kemps, 2005). However, empirical evidence for attention biases for food in restrained eaters versus healthy controls remains also inconclusive. Specifically, the direction of the attention bias is not clear yet. One study (Veenstra, de Jong, Koster, \& Roefs, 2010) used a paradigm that could distinguish between attentional approach and avoidance processes, and found evidence for an association of restrained eating scores and attentional avoidance (more disengagement from high-fat foods), whereas other studies provided evidence that restrained eaters show increased attention biases towards food stimuli when compared to unrestrained eaters (Hollitt, Kemps, Tiggemann, Smeets, \& Mills, 2010; Meule, Vögele, \& Kübler, 2012), or did not find significant differences in attention biases for food in unrestrained and restrained eaters (Ahern, Field, Yokum, Bohon, \& Stice, 2010; Boon, et al., 2000). Thus, even though evidence is mixed, research from ED patients and restrained eaters seems to suggest that (extreme) eating forbiddance might be related to differences in attention for food, yet the direction of this effect is unclear.

On the other hand, research on attention allocation in obese and overweight populations suggests that attention biases for food are related to craving, overeating and BMI. According to the theory of incentive salience, food cues can acquire motivational properties through a conditioning process: because food cues predict the rewarding experience of eating they can become salient stimuli in the environment, and are then capable of "grabbing" attention, which in turn elicits (conditioned) approach behaviour, such as craving and (over)eating of high-calorie food (Berridge, 1996, 2009; Nijs \& Franken, 2012). Thus, theoretically, attention biases for craved appetitive stimuli, such as drugs or palatable foods, have a potential role in maintaining a pattern of overconsumption (Berridge, 1996, 2009; Field, Munafó, \& Franken, 2009; Franken, 2007; Robbins \& Ehrman, 2004). Accordingly, studies have demonstrated selective processing of food cues in overweight/obese individuals when compared to healthy weight controls. Some studies found evidence for an attentional bias towards high-calorie food stimuli in the direction and duration of gaze for food versus neutral stimuli in obese but not in healthy weight participants when fed (Castellanos et al., 2009), and an attentional bias in initial orientation towards food versus neutral stimuli in overweight or obese participants (Nijs, et al., 2010; Nijs, Muris, Euser, \& Franken, 2010). However, others report an approach-avoidance reaction (initially focusing on high-calorie food followed by attentional avoidance in later stages of processing) in overweight participants (Werthmann et al., 2011).

Taken together, results suggest that there is evidence that attention biases for food cues are related to overweight and obesity (associated with craving and in- 
take of high-fat foods; i.e. high "eating motivation"), possibly apparent in attentional approach of food, as well as to (extreme) restrained eating (thus eating forbiddance in eating disordered patients and restrained eaters), possibly apparent in attentional avoidance of food. It is difficult to draw firm conclusions from previous research though, as body weight and restraint are typically confounded (e.g. Johnson, Pratt, \& Wardle, 2011; Snoek, van Strien, Janssens, \& Engels, 2008; Werthmann et al. 2013). Moreover, it is not always clear whether the observed effects reflect attentional approach or avoidance. One difficulty regarding the results of former studies is the use of different paradigms and methods to assess attention bias for food, which might have contributed to the inconsistency of previous results. More specifically, variation in the type of target (e.g., high-calorie food or different food stimuli) and the contrast category may have led to inconsistencies (Forestell, Lau, Gyurovski, Dickter, \& Haque, 2012; Werthmann, et al., 2011). Recently, a meta-analysis concluded that the most direct and immediate measure for (visual) attention biases is eye tracking technology (Field, Munafo, \& Franken, 2009). A further advantage of eye tracking is that the direction of attention, thus attention avoidance or attentional approach, can also be directly assessed. Therefore, concurrent recordings of eye movements during a visual probe task were used in the current study to determine the effect of food availability contexts on attention processing.

The current study tried to systematically disentangle the effects of eating motivation and eating forbiddance (as a proxy for (extreme) dietary restraint) on attention bias for food, using an eye-tracking paradigm that can distinguish between attentional approach and attentional avoidance processes. In this study we selected 'chocolate' as the target food, as it is both craved and seen as "forbidden" (Hetherington \& Macdiarmid, 1993; Kemps \& Tiggemann, 2009; Rodin, Mancuso, Granger, \& Nelbach, 1991), and tested how the expectancy of imminent chocolate consumption influenced attention bias for chocolate. Similarly, previous research testing whether food expectancies influence cognitive performance also used chocolate as target food (Higgs, 2007).

The main research question concerned whether the manipulation of eating expectancy within four availability contexts (required to eat, forbidden to eat, individual choice, or chance) modulates attention bias for chocolate cues, and affects craving and chocolate intake. Participants who expected to be required to eat chocolate were hypothesized to show attention biases towards chocolate cues (i.e., attentional approach), whereas participants who expected that they were forbidden to eat chocolate were hypothesized to show attention biases away from food (i.e., attentional avoidance), both in comparison to participants who could choose for themselves or who expected a $50 \%$ chance to eat chocolate. Participants in those conditions were hypothesized to show an approach-avoidance pattern of attention allocation towards chocolate stimuli (e.g., first directing attention to- 
wards chocolate but then reduced maintained attention on chocolate), because of an ambivalence conflict between temptation and self-control. Craving and consumption were supposed to be affected in the same direction as the attention biases for each condition.

To account for possible effects of individual differences in the intrinsic motivational salience of chocolate that might interfere with the effectiveness of our availability manipulation, we tested this objective in high chronic chocolate cravers compared to low cravers. Chronic chocolate craving was thought to be related to an attention bias towards chocolate cues because previous research has indicated that high chocolate cravers showed more pronounced subjective, physiological and hedonic (as marked by elevated event-related potentials in the anterior frontal scalp) reactivity when viewing chocolate images in comparison to low cravers (Asmaro et al., 2012; Rodríguez, Fernández, Cepeda-Benito, \& Vila, 2005). In addition, a manipulation check asking participants about their self-endorsed eating permission was included to account for the possibility that participants did not internalize the experimental manipulation instructions.

\section{Method}

\section{Participants}

A total of 80 female undergraduate students participated in this study. Participants were recruited via flyers, an online recruitment system and via advertisements on the university campus. Only female participants were included to obtain a homogeneous sample and to ensure comparability to previous studies on (chocolate) eating behaviour (e.g., Higgs, 2007; Kemps \& Tiggemann, 2009). For the purpose of our study participants were categorized as either high chronic chocolate cravers or low cravers based on a median split $(M d n=39.91)$ on a trait measure for chocolate craving: the subscale "chocolate craving" of the Attitude to Chocolate Questionnaire (Benton, Greenfield, \& Morgan, 1998). See Table 1 for participant characteristics. 
Table 1.

Descriptive of sample characteristics

\begin{tabular}{|c|c|c|c|c|c|c|c|c|c|c|}
\hline \multirow[b]{3}{*}{$\begin{array}{l}\text { Participant } \\
\text { characteristics }\end{array}$} & \multirow{2}{*}{\multicolumn{2}{|c|}{$\begin{array}{l}\text { Overall } \\
\text { Total sample } \\
(N=80)\end{array}$}} & \multicolumn{4}{|c|}{ Craving status } & \multicolumn{4}{|c|}{ Self-endorsed eating permission } \\
\hline & & & \multicolumn{2}{|c|}{$\begin{array}{l}\text { High chocolate } \\
\text { cravers } \\
(n=40)\end{array}$} & \multicolumn{2}{|c|}{$\begin{array}{l}\text { Low chocolate } \\
\text { cravers } \\
(n=40)\end{array}$} & \multicolumn{2}{|c|}{$\begin{array}{l}\text { Lack of } \\
\text { permission } \\
(n=20)\end{array}$} & \multicolumn{2}{|c|}{$\begin{array}{l}\text { Permission } \\
(n=60)\end{array}$} \\
\hline & $M$ & $S D$ & $M$ & $S D$ & $M$ & $S D$ & $M$ & $S D$ & $M$ & $S D$ \\
\hline $\begin{array}{l}\text { Trait chocolate } \\
\text { craving (ACQ) }\end{array}$ & 39.67 & 17.48 & $53.83^{\mathrm{a}}$ & 10.17 & $25.51^{\mathrm{a}}$ & 10.23 & 34.75 & 15.17 & 41.31 & 18.01 \\
\hline $\begin{array}{l}\text { Last chocolate } \\
\text { consumption(hr) }\end{array}$ & 40.79 & 51.49 & $25.70 \mathrm{a}^{*}$ & 27.51 & $57.38 \mathrm{a}^{*}$ & 65.50 & 55.59 & 83.57 & 35.74 & 34.57 \\
\hline $\begin{array}{l}\text { Average } \\
\text { chocolate } \\
\text { consumption per } \\
\text { week (cal) }\end{array}$ & 202.36 & 172.62 & $239.67^{+}$ & 169.66 & $150.14^{+}$ & 166.99 & 153.27 & 129.87 & 216.96 & 182.39 \\
\hline $\begin{array}{l}\text { Dietary } \\
\text { Restraint (RS) }\end{array}$ & 11.69 & 3.33 & 12.22 & 3.56 & 11.16 & 3.03 & $10.45 b$ & 4.03 & $12.10_{b}$ & 2.98 \\
\hline BMI & 22.20 & 1.79 & 22.25 & 1.86 & 22.16 & 1.73 & 22.34 & 1.97 & 22.15 & 1.74 \\
\hline
\end{tabular}

Note. $\mathrm{ACQ}=$ Attitudes to Chocolate Questionnaire; RS = Restraint Scale; BMI = Body Mass Index.

Superscript letter (a) indicates differences between high chronic chocolate cravers and low cravers at $\mathrm{p}<.05$.

Subscript letter (b) indicates a trend for a significant differences between participants with and without self-endorsed eating permission $(\mathrm{p}=.054)$

* comparison based on $\mathrm{df}=61$, because fifteen participants did, by mistake, not receive these questions. Three participants did not answer the question regarding the time of their last chocolate consumption, suggesting that they either never ate chocolate or could not answer the question.

+ comparison based on $\mathrm{df}=46$, because fifteen participants did, by mistake, not receive these questions. 17 participants did not answer the question regarding their average consumption, suggesting that they either never ate chocolate or could not answer the question.

\section{Materials and Procedure}

Craving Exposure. To ensure that all participants regarded chocolate, at least momentarily, as "desirable" food item, a chocolate exposure, focusing on palatable features of chocolate, was conducted at the start of the experimental session. Participants were instructed to smell a piece of chocolate intensively (of their choice from a bowl filled with different pieces of chocolate) for 3 minutes. During this time participants were encouraged to indulge in the experience of their craving by concentrating on different sensual aspects, such as smell and texture of the chocolate pieces, without tasting it. A booster for chocolate craving with a shorter 
(1 min) similar exposure to chocolate was inserted in a short break during the attention paradigm (i.e. the visual probe task).

Manipulation of eating expectancy. Perceived eating opportunity was manipulated in four conditions and participants were randomly, with the restriction of equal group sizes, assigned to one of these conditions. Participants received one of the following instructions: (1) consumption is required (required), (2) consumption is not allowed (forbidden), (3) you can choose whether you want to eat chocolate (choice), or (4) consumption is determined by the roll of a dice (chance).

Pictorial visual probe paradigm.

Overview. Attention allocation towards chocolate was measured with a visual probe task. During this task two image pairs were presented simultaneously side by side followed by a probe $\left({ }^{*}\right)$ appearing in the location of one of the images. Participants were instructed to respond as quickly as possible by pressing a corresponding key on a button box to indicate the location of the probe (response latency was registered), while their eye movements during the stimulus presentation were measured, as a direct measure of attention allocation. The complete task was divided into 2 blocks of 60 trials each (total of 120 trials). A break was inserted between these blocks, in which participants were again exposed to the smell of chocolate and the respective instruction. The task included 80 critical trials and 40 filler trials.

Timing Trials. Each trial started with a central fixation cross, which remained on the screen for $100 \mathrm{~ms}$ when participants focussed on the cross. Subsequently, the target image pair was presented for $3000 \mathrm{~ms}$. Then the probe, $\left({ }^{*}\right)$, was presented until the participant responded by pressing a key on the button box.

Trial types. Critical trials consisted of 20 stimulus pairs, which were each presented four times. In critical trials, the image pair consisted of a picture of a chocolate item and a picture of a musical instrument. Music instruments were used to obtain a close match of colour (mostly brownish for chocolate items and music instruments alike). Filler trials consisted of 10 stimulus pairs, which were also each presented four times. Filler trials consisted of picture pairs depicting two neutral non-food photographs (office supplies and traffic objects). All image pairs were matched as closely as possible with regard to colour, complexity, brightness, and size. The position of the probe was equally distributed per stimulus type and equally often o then left and right side of the screen. The order of trials was randomized uniquely for each participant.

Eye movement measurements. Eye movements were recorded by a desktop mounted EyeLink 1000 system (SR Research Ltd., Mississauga, Ontario, Canada). A 9-point calibration with subsequent validation procedure was conducted prior to the visual probe paradigm. To assess attention allocation processes, participants' gaze fixations were analysed. Gaze fixations were defined as any period that is not 
a blink or saccade and lasted at least 100 ms (Eyelink Dataviewer User's Manual, 2002-2008, SR Research Ltd.). Eye movements that occurred before the presentation of an image pair were excluded, because these movements could represent anticipatory fixations.

For analyses purposes, the computer screen was, invisible for participants, divided in three areas of interest: the mid-section, which indicates the location of the fixation cross, and the left and right section, representing the locations of the picture stimuli. Only eye movements in critical trials directed either to the left or the right section of the screen were extracted for further analyses. Eye movements in filler trials and gaze fixations in the mid area were excluded from further analyses. Eye movements were extracted using Data Viewer (SR Research Ltd., Mississauga, Ontario, Canada).

Attention Bias Scores. Three attention bias scores were derived from the eye movement data: gaze direction bias, initial gaze duration bias and dwell time bias. A gaze direction bias is described as a measure for initial orientation towards relevant stimuli. The gaze direction bias was based on the proportion of trials in which the first fixation was directed to a chocolate stimulus instead of a neutral control stimulus. A bias score greater than 50\% represents a higher proportion of first fixations directed to chocolate stimuli, whereas a bias score less than $50 \%$ indicates a higher proportion of first fixations directed to neutral stimuli.

Initial gaze duration bias is the sum of multiple fixations occurring within the region of the initially fixated picture before gaze is shifted away from it and can be seen as a measure for early attention maintenance (e.g. Mogg, Bradley, Field, \& De Houwer, 2003). Bias scores for the initial gaze duration were computed by subtracting the mean duration of initial fixations directed to neutral images from the mean duration of initial fixations directed to chocolate images. Thus, a positive score is indicative of longer initial attention maintenance on chocolate stimuli, whereas a negative score is indicative of the reverse: longer initial maintenance on neutral stimuli.

Dwell time bias is informative regarding the maintenance of attention on critical stimuli (e.g. Mogg, Field, \& Bradley, 2005). Overall dwell time per image category (chocolate vs. neutral) was calculated for each critical trial, and then averaged per image category over all trials, resulting in an average total dwell time per image category. For the gaze dwell time bias score, the mean dwell time on neutral images was subtracted from the mean dwell time on chocolate images. Thus, a positive score indicates that attention was maintained longer on chocolate items than on neutral items, whereas a negative score indicates the reverse: longer maintained attention on neutral items.

Manual response latencies to probes. Calculations of response latency bias scores were based on the recordings of the participant's manual response latency when indicating the location of the probe. Response latencies were excluded from 
further analyses if they were faster than $200 \mathrm{~ms}$, slower than 2000 , and then if they deviated more than 3 SDs from each participant's mean (e.g. Castellanos, et al., 2009; Mogg, Bradley, Hyare, \& Lee, 1998). Bias scores were then calculated by subtracting the mean response latency on trials when the probe replaced a chocolate image (i.e. chocolate - congruent trials) from the mean response latency on trials when the probe appeared in the same location as the preceding neutral image (i.e. chocolate - incongruent trials). A positive bias score can be interpreted as an attention bias towards chocolate, whereas a negative bias score indicates an attention bias away from chocolate, thus attentional avoidance.

\section{Questionnaires}

State chocolate craving. State craving for chocolate was assessed by a 100 $\mathrm{mm}$ visual analogue scale (VAS) indicating "How strong is your desire for chocolate right now?" ( $0=$ I have no desire for chocolate at all at the moment; $100=$ I have an extreme desire for chocolate at the moment"). Participants were asked to fill in this VAS at four successive time points during the experimental procedure: when entering the lab (VAS1); after the exposure procedure (VAS2); after the booster exposure during the break of the visual probe task (VAS3); shortly before the taste test (VAS4).

Trait chocolate craving. Trait chocolate craving was assessed with the "Attitudes to Chocolate Questionnaire" (ACQ; Benton, et al., 1998). This questionnaire can be divided into three subscales (chocolate craving, guilt for chocolate, functional approach to chocolate eating) and mean subscale scores can be calculated. Chocolate craving is assessed on 24 items measuring the characteristics of typically experienced craving for chocolate on $100 \mathrm{~mm}$ VAS ranging from "Not at all like me" (0) to "Very much like me" (100). Higher scores are indicative of stronger trait chocolate craving (Benton, et al., 1998).

Habitual Chocolate Consumption. Participants were asked to indicate their estimated average chocolate consumption per week and the time passed since they last ate chocolate with two open ended questions.

Restraint Scale (RS; Herman \& Polivy, 1980). To characterize the participant groups, restrained eating style was assessed by means of the restraint scale. This 10 item self report questionnaire assesses weight concerns, weight fluctuations and self-reported attempts to diet, on a scale ranging from 0 (concerns absent) to 3 or 4 (concerns present); with higher scores indicating more concern for weight and difficulties with control over eating behaviour.

Body Mass Index (BMI). Height and weight were measured at the end of the experimental session to calculate BMI. 
Taste Test

Chocolate intake was measured by means of a bogus "taste test". The participant was instructed to rate four bowls (mean weight $=762.97 \mathrm{~g}$, SD $=50.01$ ) of chocolate pellets (white chocolate, milk chocolate, $53.8 \%$ cacao dark chocolate, $70.3 \%$ cacao dark chocolate) on visual attractiveness, smell, and taste while the experimenter left the room for 10 minutes. The instruction procedures for the "forbidden" and for the "chance" condition were slightly adjusted to avoid contradictions with the expectancy instruction they received earlier. In condition 4 ("Chance") the participant was informed that there would be a chance that they had to eat chocolate; yet the chance element (i.e. a digital dice) was manipulated in a way that all participants received the instruction to eat chocolate. For participants in condition 2 ("Forbidden") the instruction for the taste test included the adjunct "You will not really be eating chocolate, however, you will be participating in a taste test about chocolate." followed by the standard instruction "You can try as much as you want, to be able to judge the different sorts of chocolate adequately". Chocolate intake was determined by difference in weight of bowls before and after the taste test.

\section{Manipulation check}

To check whether the manipulation instructions about eating opportunity produced indeed different perceptions and feelings towards chocolate consumption a short manipulation check was conducted at the end of the experiment. Participants were probed for suspicion: Participants were asked to identify the correct instruction that they received among all available instructions to check whether the instructions were understood (and remembered) well by participants. Then, all participants were asked to answer the question "Were you allowed by yourself to eat chocolate?", to see to what extent they internalized the instructions they received. This measure was later used in analyses to account for the possibility that participants did not internalize the experimental manipulation instructions and to explore how self-endorsed eating permission contributed to differences in attention, craving or intake.

\section{Procedure}

All participants provided informed consent prior to participation and each participant was tested individually. Participants were tested between 11 am and $5 \mathrm{pm}$. Upon arrival at the laboratory participants filled in VAS1 to indicate baseline craving for chocolate and were then subjected to 3 minutes of chocolate exposure to cause an increase in chocolate craving, and reported their craving after the exposure on VAS2. Subsequently, participants received instructions about their eating opportunity according to their condition (required/forbidden/choice/chance). Participants then conducted the visual probe task while eye movements towards chocolate versus non-chocolate stimuli and manual response latencies were rec- 
orded as measures of attention biases for chocolate. In the short break during this task the participant again underwent a brief (1 $\mathrm{min}$ ) exposure to chocolate to 'boost' the craving, and the respective chocolate availability instruction was repeated. At the end of the short break, participants reported their craving for chocolate on VAS3 before continuing with the second part of the visual probe task. After the visual probe task, participants again indicated their craving for chocolate on VAS4 and subsequently took part in the bogus taste test. Participants then filled in the questionnaires (trait chocolate craving measure (ACQ: Benton, et al., 1998); dietary restraint (RS: Herman \& Polivy, 1980); manipulation check) and height and weight were measured. Participants were thanked at the end of the procedure and received either course credit of a gift certificate of $€ 7,50$ for their participation. The current study was reviewed and approved by the local ethics committee.

\section{Results}

\section{Chocolate craving}

Changes in self-reported momentary craving were analysed in a full factorial mixed model analysis of variance (ANOVA) with time (4 time points) as a within subjects factor, and condition (required, no consumption, choice, chance), and chocolate craving status (high chocolate craver vs. low craver) as between subjects factors. Overall, craving was significantly different over the four time points, as was evident from a main effect of time, $F(2.31,166.09)^{3}=112.92, p<.0001$. High chronic chocolate cravers reported generally higher levels $(M=67.70, S D=17.06)$ of momentary craving than did low cravers $(M=45.98, S D=17.06)$, as evidenced by a main effect of chocolate craver status, $F(1,72)=31.87, p<.001$. None of the other effects in the ANOVA reached significance, all $F \mathrm{~s}<2.00$, all $p s>.13$. Pairwise comparisons revealed a significant increase in chocolate craving at time $2(M=$ $59.94, S D=2.08 ; p<.0001)$, time $3(M=65.84, S D=2.11 ; p<.0001)$ and time $4(M=$ $62.21, S D=2.3 ; p<.0001)$ in comparison to craving at time $1(M=39.36, S D=$ 2.12).

\section{Attention biases}

To test whether attention bias scores differed between conditions and to account for chronic chocolate craving separate full factorial ANOVAs were conducted for each measure of attention bias (direction bias, initial gaze duration bias, dwell time bias, response latency bias) with condition (required, no consumption, choice,

\footnotetext{
${ }^{3}$ Assumption of sphericity has been violated, therefore Greenhouse-Geisser correction are reported.
} 
chance) and chocolate craver status (high chocolate craver, low craver) as between subject variables.

Results showed a main effect of chronic chocolate craver status on two measures of attention bias: High chronic chocolate cravers had significantly higher initial gaze duration bias scores, $F(1,72)=7.70, p=.007$, and marginally significantly higher dwell time scores than low cravers, $F(1,72)=3.61, p=.062$, see Figure 1. No other effects in the ANOVAs on initial gaze duration bias and dwell time reached significance, all $F \mathrm{~s}<1.56$, all $p \mathrm{~s}>.21$ (for initial fixation duration) and all $F s<0.77$, all $p s>.51$ (for dwell time bias). The ANOVAs on direction bias and on response latency bias revealed no significant effects at all, all $F \mathrm{~s}<1.86$, all $p \mathrm{~s}>.14$ (for direction bias) and all $F_{\mathrm{S}}<2.38$, all $p s>.13$ (for response latency bias).

Additional one sample t-tests were conducted to test whether chocolate cues attracted more attention than neutral stimuli, overall. A one sample $t$-test against 50 (which indicates no direction bias) revealed that all participants directed their first gaze on average significantly more often than chance towards chocolate stimuli than towards neutral stimuli, $t(79)=8.10, p<.001$. In addition, all participants looked longer towards chocolate than neutral stimuli in later attention processes, as revealed by elevated bias scores in one-sample t-tests against 0 (indicating no bias) for initial gaze duration bias $(t(79)=6.46, p<.001$ and dwell time bias $(t(79)$ $=6.46, p<.001$ ). Similarly, all participants had a significantly elevated response latency bias, indicating faster response latencies when a probe replaced a chocolate stimulus in comparison to neutral stimulus, $t(79)=4.94, p<.001$, as qualified by a one sample t-test against 0 (indicating no bias in response latencies). In sum, results of one sample t-tests showed that all participants generally expressed elevated approach attention processes for chocolate stimuli vs. neutral stimuli on all measured attention bias scores.

\section{Chocolate intake}

To test whether the expectancy to eat chocolate or whether chronic chocolate craving affected food intake, a full factorial ANOVA on chocolate intake (in calories) with condition (required, no consumption, choice, chance) and chocolate craver status (high chronic chocolate craver vs. low craver) was conducted. Results indicated a marginally significant main effect of chronic chocolate craving on chocolate intake. High chronic chocolate cravers tended to consume significantly more chocolate than low cravers, $F(1,72)=3.84, p=.054$. See Figure 1 . 


\section{Subjective permission to eat chocolate}

Although attention bias and chocolate intake were not affected by the experimental manipulation of eating expectancy (i.e. externally controlled permission to eat), we also explored whether attention biases for chocolate and chocolate intake were associated with subjective (self-endorsed, thus internalized) permission to eat. Based on the question if participants permitted themselves to eat chocolate (yes/no) two groups were created: participants who reported that permission to eat chocolate was not self-endorsed $(n=20)$ and participants whose permission to eat chocolate was self-endorsed $(n=60)^{4}$. The effect of self-endorsed permission to eat was tested by means of independent samples $t$-tests with the attentional bias measures (four bias measures), self-reported craving (at four time points) and chocolate intake (in calories) as the dependent variables. Results revealed that participants who reported a lack of self-endorsed eating permission had significantly lower craving ratings throughout the experiment, all $t \mathrm{~s}>2.49$, all $p \mathrm{~s}<.02$, see Table 2, and significantly lower dwell time bias scores, $t(78),=2.01, p<.05$, in contrast to participants whose permission to eat was self-endorsed. No significant differences between these groups emerged with regard to other attention bias scores, all $t \mathrm{~s}<1.53$, all $p s>.13$ ) or food intake, $t(78)=1.00, p=.32$. See Figure 1 .

Figure 1. Mean attention bias scores (direction bias (in \%), initial gaze duration, total dwell time and response latency bias (all in ms)) and chocolate intake (in calories) for high chronic chocolate cravers (black column) and low cravers (white column) (left panel) and for participants who reported that they would not have permitted themselves to eat chocolate (black column) and participants reported that they permitted themselves to eat chocolate (white column) (right panel).

Note. $P$-values are reported based on estimated marginal means, for chocolate craver and non-cravers, and on raw means for participants with and without self-endorsed permission to eat.

\footnotetext{
${ }^{4} \mathrm{~A}$ significant $\chi^{2}$-test between conditions indicated that the conditions differed significantly regarding the number of participants who indicated self-endorsed eating permission ( $p$-value of $\chi^{2}=.028$ ): In the "chance" condition, only one participant indicated that she did not self-endorse eating permission, yet self-endorsed eating permission did not differ between participants in the "required to eat", "forbidden" or "choice" condition ( $p$-value of $\chi^{2}=.231$ ). Results remained the same when analyses were repeated including only participants of the required/forbiddance/choice condition. Results reported here are based on the data of all participants.
} 


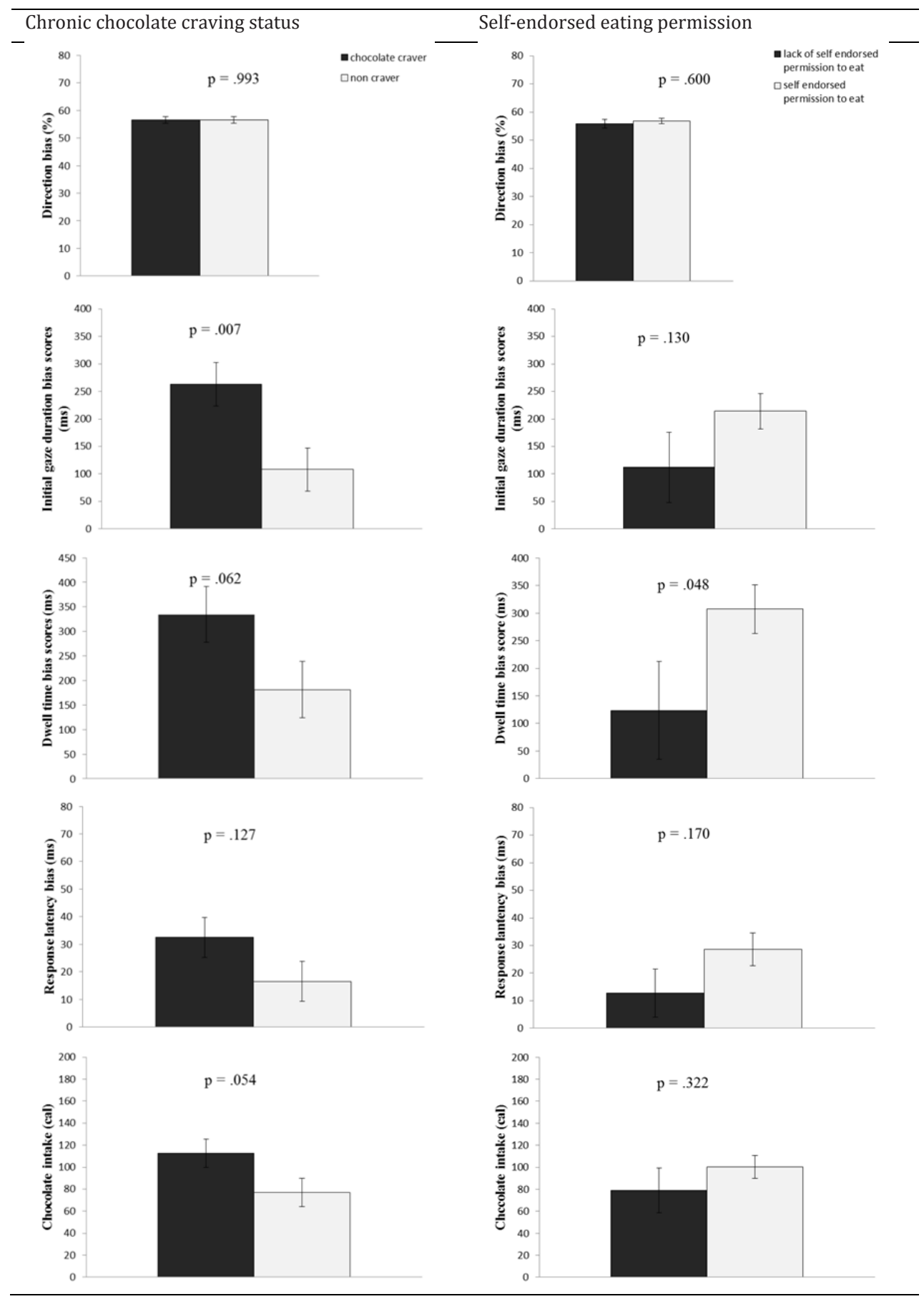


Table 2.

Self-reported craving in participant with and without self-endorsed eating permission at four timepoints.

\begin{tabular}{llllll}
\hline & \multicolumn{2}{l}{ Lack of permission $(n=20)$} & & \multicolumn{2}{l}{ Permission $(n=60)$} \\
\cline { 2 - 3 } \cline { 5 - 6 } Self-reported Craving & $M$ & $S D$ & & $M$ & $S D$ \\
\hline Craving at t1 & 28.90 & 23.74 & & 42.91 & 21.10 \\
Craving at t2 & 45.85 & 21.26 & & 64.80 & 19.17 \\
Craving at t3 & 52.55 & 22.62 & & 70.27 & 17.57 \\
Craving at t4 & 47.75 & 24.06 & 67.03 & 19.28 \\
\hline
\end{tabular}

Note. Craving reports were significant different between groups at all four timepoints $(p<.05)$.

\section{Correlations of craving, attention biases and chocolate intake}

In the whole sample, both initial gaze duration bias and dwell time bias correlated with craving at all time points (all $r s(80)>.29$, all $p s<.01$ for initial gaze duration; all $r \mathrm{~s}(80)>.28$, all $p \mathrm{~s}<.01$ for dwell time bias). Correlation analyses further showed that chocolate intake was also significantly correlated with all four measures of attention biases. See Table 3 .

Table 3.

Pearson correlation between four measures of attention bias (direction bias, initial gaze duration bias, dwell time bias and response latency bias) and chocolate intake (in cal.).

\begin{tabular}{|c|c|c|c|c|c|c|c|c|c|}
\hline Variable & 1 & 2 & 3 & 4 & 5 & 6 & 7 & 8 & 9 \\
\hline 1. Direction bias & $\begin{array}{l}56.58 \\
(7.26)\end{array}$ & & & & & & & & \\
\hline $\begin{array}{l}\text { 2. Initial gaze duration } \\
\text { bias }\end{array}$ & .20 & $\begin{array}{l}188.74 \\
(261.26\end{array}$ & & & & & & & \\
\hline 3. Dwell time bias & $.29 *$ & $.86^{*}$ & $\begin{array}{l}261.15 \\
(360.61)\end{array}$ & & & & & & \\
\hline 4. Response latency bias & .03 & $.38^{*}$ & $.45^{*}$ & $\begin{array}{l}24.64 \\
(44.65)\end{array}$ & & & & & \\
\hline 5. Chocolate intake (cal). & $.27^{*}$ & $.25^{*}$ & $.33^{*}$ & $.35^{*}$ & $\begin{array}{l}94.96 \\
(82.45)\end{array}$ & & & & \\
\hline 6. Craving at time 1 & -.04 & $.29 *$ & $.28^{*}$ & .13 & $.31^{*}$ & $\begin{array}{l}39.41 \\
(22.48)\end{array}$ & & & \\
\hline 7. Craving at time 2 & -.06 & $.36^{*}$ & $.35^{*}$ & .19 & $.33^{*}$ & $.79 *$ & $\begin{array}{l}60.06 \\
(21.24)\end{array}$ & & \\
\hline 8. Craving at time 3 & -.03 & $.39 *$ & $.40^{*}$ & $.30 *$ & $.42^{*}$ & $.70^{*}$ & $.92 *$ & $\begin{array}{l}65.84 \\
(20.33)\end{array}$ & \\
\hline 9. Craving at time 4 & .11 & $.35^{*}$ & $.34^{*}$ & .21 & $.48^{*}$ & $.72^{*}$ & $.80^{*}$ & $.82^{*}$ & $\begin{array}{l}62.21 \\
(22.08)\end{array}$ \\
\hline
\end{tabular}

Note. Off-diagonal shows correlation coefficients; Diagonal shows means and standard deviations.

* Correlations are significant $(p<.05$, two tailed). 


\section{Discussion}

Attention biases for food have been associated with (extreme) dieting as well as with (chronic) craving and eating enjoyment. The aim of this study was to systematically examine the effects of eating motivation and food forbiddance on attentional bias for food, momentary craving, and food intake during four experimentally manipulated availability contexts. Our results showed that the manipulation of (perceived) availability of chocolate did not influence participants attention, momentary craving or chocolate intake, which leads to the conclusion that this manipulation did not affect the eating motivation of participants. Maybe short-termed manipulation of anticipated consumption does not affect attention to a similar extent as trait-like variables, such as chronic chocolate craving. This is in line with previous findings, showing that an experimental instruction to manipulate anticipated consumption did not modulated cognitive processing in participants (Higgs, 2007). To strengthen the effect of the availability manipulation the integration of direct reinforcement of the respective manipulation instructions on a trial-by-trial basis could possibly be helpful, as for example, has successfully been applied in recent studies on alcohol and reward expectancies (Field et al., 2011; Jones et al., 2012).

In general, the current findings indicate that chocolate stimuli attracted more attention than neutral stimuli, because all participants paid more attention to chocolate cues than to neutral cues, as was evident for all attention bias scores (i.e., for direction bias, initial gaze duration bias, dwell time bias and response latency bias). This finding might not be entirely suprising because noticing food stimuli has always been important for our survival and food cues might therefore inherently possess more motivational salience than neutral stimuli.

More importantly, results also confirm that attention biases for food were related distinctly to chronic craving and self-endorsed permission to eat. Specifically, chronic chocolate craving was related to elevated approach attention biases for chocolate cues in later stages of attention allocation, that is, the duration of initial gaze and (marginally) total dwell time on chocolate stimuli. In contrast, total dwell time on chocolate was significantly reduced in participants who subsequently indicated that they would not have permitted themselves to eat chocolate (i.e., those with lack of self-endorsed eating permission).

These effects on attention allocation for chocolate cues were only apparent in a more controlled and later stage of attention processing, in this study. No distinctive effects of chronic craving or self-endorsed eating permission on attention for chocolate cues were observed on early/automatic (direction bias) and indirect (response latency bias) attention measures. These findings of attention biases in later processing stages match with results of basic attention research showing that goal motivation steers voluntary attentional processing 
(Vogt, De Houwer, \& Crombez, 2011) and contribute further to the suggestion that particularly the maintenance of attention mirrors top-down attentional control processes, that take longer and reflect motivation (LaBerge, 2002). In this respect, it is interesting to note that specifically the duration measures of attention were positively related to craving and chocolate consumption in our study. Maybe prolonged attention maintenance, as studied here, is an expression of heightend eating motivation.

Even though speculative at this point, because of the correlational nature of our findings, this might further suggest that attention biases towards food cues could function as a maintenance factor for overeating. If individuals with chronic craving for food spend more time looking at food and if this bias is related to their food consumption, attention biases for food might be particularly problematic in an obesogenic food environment with highly salient and tempting food cues. In this respect, our findings support evidence from previous studies that reported approach attention bias for food cues in obese, in hungry and in craving participants (elevated eating motivation for food, e.g.; Castellanos, et al., 2009; Piech, Pastorino, \& Zald, 2010; Smeets, Roefs, \& Jansen, 2009).

Whereas high intrinsic eating motivation (i.e., chronic chocolate craving) was associated with attentional approach for chocolate cues, our results also showed that a lack of self-endorsed eating permission was associated with a diminished attention bias for chocolate cues. One possible explanation is that a lack of selfendorsed eating permission leads to both lower craving and reduced attention of chocolate cues. Or, the effect of self-endorsed eating permission on reduced attention may be mediated by experienced craving. However, the reverse chain of events may also be possible, as another explanation can be that decreased craving at baseline led both to a lack of self-endorsed eating permission and diminished attention for chocolate.

At this point, these explanations remain speculative because it is not possible to statistically disentangle the temporal order of craving reports and reports of self-endorsed eating permission ${ }^{5}$. Thus, results concerning the possible influence of self-endorsed eating permission on attention and craving should be viewed under this limitation and a replication of our results under experimental conditions is warranted. An implication of this finding could be that individuals who follow strict dieting rules (or who have less intrinsic motivation to eat) pay less attention to food cues and experience less cravings. In this respect, our finding dovetails previous evidence hinting at attentional avoidance of food cues in eating disorder patients and restrained eaters (Brooks, et al., 2011; Shafran, et al., 2007, 2008; Veenstra, et al., 2010). Moreover, our results also correspond with recent

\footnotetext{
${ }^{5}$ We measured self-endorsed eating permission only once, at the end of the experiment, but craving was measured consecutively throughout the experiment. Therefore we do not have data that informs on the temporal order of craving or eating permission.
} 
findings of an eye tracking study showing attentional avoidance of food cues, only in later stages of attentional processing, in anorexic patients but not in healthy participants (Giel et al., 2011). In addition, this finding also corroborates previous research indicating that highly restrained eaters down-regulate their hedonic response to food, when food is available (Blechert, Feige, Hajcak, \& Tuschen-Caffier, 2010). In general, our findings that differences in chronic craving and eating permission result in different attention allocation patterns fits with recent neuroimaging studies showing that attention focus (e.g. focussing on palatability versus view freeing) modulates neuro-cognitive reactivity for food in healthy weight and overweight samples (Frankort et al., 2012; Siep et al., 2009).

In conclusion, this is the first study showing the differential effect of chronic craving and self-endorsed eating permission on the strength and the direction of attention biases for food cues by means of eye-tracking. Results suggest that chocolate cues, in comparison to neutral cues, are highly salient stimuli in our environment and generally attract more attention than neutral cues. Moreover, chronic craving and self-endorsed eating permission determine the extent and the time we keep focussing on food: The voluntary decision to refrain from eating chocolates was related to reduced attention for chocolate whereas chronic chocolate craving was related to attentional approach of chocolate cues. 



\section{Chapter 6}

\section{Looking at food in sad mood: \\ Do attentional biases lead emotional eaters into overeating after negative mood induction?}

Werthmann, J., Renner F., Roefs, A. Huibers, M., Plumanns, L., Krott, N., \& Jansen, A. (in press). Looking at food in sad mood: do attentional biases lead emotional eaters into overeating after negative mood induction? Eating Behaviours. 


\section{Abstract}

Background: Emotional eating is associated with overeating and the development of obesity. Yet, empirical evidence for individual (trait) differences in emotional eating and cognitive mechanisms that contribute to eating during sad mood remain equivocal.

Aim: The aim of this study was to test if attention bias for food moderates the effect of self-reported emotional eating during sad mood (vs neutral mood on actual food intake. It was expected that emotional eating is predictive of elevated attention for food and higher food intake after an experimentally induced sad mood and that attentional maintenance on food predicts food intake during a sad versus a neutral mood.

Method: Participants ( $\mathrm{N}=85)$ were randomly assigned to one of the two experimental mood induction conditions (sad/neutral). Attentional biases for high caloric foods were measured by eye tracking during a visual probe task with pictorial food and neutral stimuli. Self-reported emotional eating was assessed with the Dutch Eating Behavior Questionnaire (DEBQ) and ad libitum food intake was tested by a disguised food offer.

Results: Hierarchical multivariate regression modeling showed that selfreported emotional eating did not account for changes in attention allocation for food or food intake in either condition. Yet, attention maintenance on food cues was significantly related to increased intake specifically in the neutral condition, but not in the sad mood condition.

Discussion: The current findings show that self-reported emotional eating (based on the DEBQ) might not validly predict who overeats when sad, at least not in a laboratory setting with healthy women. Results further suggest that attention maintenance on food relates to eating motivation when in a neutral affective state, and might therefore be a cognitive mechanism contributing to increased food intake in general, but maybe not during sad mood.

Keywords: Attention bias, eye-tracking, emotional eating, mood induction, food intake 
Overeating is a serious problem, as it contributes to the development of obesity (e.g., Hill, Catenacci, \& Wyatt, 2005; Lowe \& Levine, 2005). Therefore it is important to study (cognitive) mechanisms that are related to overeating (Lowe \& Levine, 2005). Different models emphasize the impact of negative emotions on excessive food intake, thereby linking emotional eating to overeating and ultimately to the risk of weight gain (e.g., Canetti, Bachar, \& Berry, 2002; Macht, 2008). Basically, it is assumed that eating in response to stress or sad mood is a maladaptive emotion regulation mechanism, which affects eating behavior and weight in general (Canetti, et al., 2002; Macht, 2008). For example, emotional eaters, that is, individuals who report to be prone to overeat in response to negative emotional states, are thought to be less successful in dieting and are therefore considered to be at higher risk for developing obesity (van Strien, 2005). Yet, experimental research findings remain inconsistent with respect to the influence of negative mood on eating behavior in self-reported emotional eaters, as assessed with a standard self-report measure for emotional eating, the Dutch Eating Behavior Questionnaire (DEBQ, van Strien, Frijters, Bergers, \& Defares, 1986). Even basic assumptions, for example that self-reported emotional eating is associated with actual overeating during sad mood (thus with the very behavior that this construct is based upon) could not reliably be demonstrated. Whereas some experimental studies found that food intake after a negative mood induction increased in participants with high disinhibition tendencies and/or high self-reported emotional eating (Loxton, Dawe, \& Cahill, 2011; Yeomans \& Coughlan, 2009), others did not find an influence of emotional eating traits on food intake during distress in the lab (e.g. Evers, de Ridder, \& Adriaanse, 2009; Evers, Marijn Stok, \& de Ridder, 2010; Wallis \& Hetherington, 2004; 2009, study 2). Similarly, Bongers and her colleagues (Bongers, Jansen, Havermans, Roefs, \& Nederkoorn, 2013) reported that emotional eating (measured with the DEBQ) was significantly related to higher intake after positive, but not after a negative mood induction. In contrast, van Strien and her colleagues (van Strien et al., 2013) found increased intake in high emotional eaters compared to low emotional eaters after negative and after positive mood induction. Moreover, also findings from "outside the lab", for example based on food diaries, failed to find a consistent link of self-reported emotional eating and food intake during distress in real-life settings (Adriaanse, de Ridder, \& Evers, 2011; Ayres, Prestwich, Conner, \& Smith, 2011; Conner, Fitter, \& Fletcher, 1999; Wallis \& Hetherington, 2009, study 1).

A goal of the current study was to study if self-reported emotional eating is related to elevated attention bias for food and actual food intake during sad mood (vs neutral mood). Research in addiction and eating context has already shown that elevated attention processing of desired cues (i.e. attention bias) is related to craving and consumption (Field, Munafó, \& Franken, 2009; Franken, 2007; Nijs, Franken, \& Muris, 2009). Correspondingly, several studies have shown that elevat- 
ed attentional biases for high-calorie foods are related to a higher reward drive, hunger, (chronic) craving, overweight and obesity (Castellanos et al., 2009; Mogg, Bradley, Hyare, \& Lee, 1998; Tapper, Pothos, \& Lawrence, 2010; Jessica Werthmann, Roefs, Nederkoorn, \& Jansen, 2013; Jessica Werthmann et al., 2011). Thus, attention bias might be a cognitive mechanism that indicates food-related motivation and contributes to food intake. Therefore, we aimed to test if selfreported emotional eating is related to elevated attention bias for food during sad mood. Attention bias for food might be a more sensitive measure to capture foodrelated motivation in the laboratory, in contrast to actual food intake. Moreover, attention bias for food could also be a moderator of food intake in emotional eaters after a negative mood induction. In addition, it was examined if prolonged attention for food relates to increases in food calorie intake and if this relation is affected by mood. Particularly the attention maintenance component has been associated with motivational top-down processing and might therefore be a good indicator for eating motivation (LaBerge, 2002). Specifically, previous research examining the link of attention for appetitive cues and appetitive behavior indicated that particularly attentional maintenance on appetitive cues was associated with differences in motivation for the appetitive cue (Brignell, Griffiths, Bradley, \& Mogg, 2009; Field, Mogg, Zetteler, \& Bradley, 2004; Field, et al., 2009; Jessica Werthmann, et al., 2013). Thus, to further test the importance of attention bias for emotional eating, we also aimed to explore if attentional maintenance is a better predictor of overeating during sad mood than self-reported emotional eating.

In sum, measuring attention bias for food during sad mood could serve two purposes: attentional biases for food during sad mood might be less controllable than actual eating behavior during a taste test, and might therefore offer a more sensitive, less obtrusive measure of increased eating motivation during sad mood than actual food intake in the lab for emotional eaters. In addition, assessing attentional biases for food during sad mood could provide us with more knowledge on the cognitive working mechanisms that lead to increased food intake during sad mood.

Research that examined if attention for food is related to self-reported emotional eating is sparse. Hepworth and colleagues (2010) observed that during a sad mood induction a response-latency based measure of attention bias for food and self-reported appetite increased. Moreover, this bias correlated positively with emotional eating and the DEBQ score overall, however in a regression analysis the interaction term of emotional eating and experimental mood induction was not predictive for attention. Yet, direct food intake was not assessed in this study and attention biases for food were measured indirectly through reaction times. Thus, this previous result questions the link of emotional eating, attention bias for food and food intake, yet experimental evidence for this relation is still lacking and 
leave therefore the exploration of cognitive mechanisms contributing to overeating in emotional eaters during sad mood unresolved.

With regard to the measurement of attention bias we extended this previous finding by employing eye tracking, which has been found to be the most sensitive and direct way to test individual differences in attention allocation in the domain of addiction (Field, et al., 2009). Moreover, we made use of pictures instead of words as stimuli during the visual probe task, because it has been argued that pictures are more ecologically valid for the assessment of visual attention for appetitive cues (e.g. Brignell, et al., 2009; Hepworth, et al., 2010). Thus, this is, to our knowledge, the first study to test the impact of mood on attentional biases experimentally by using eye-tracking as novel and dynamic measure of visual attention allocation for pictorial food stimuli.

To summarize, the aims of this study were twofold: first, it was tested if selfreported emotional eating is related to increased attention bias for food and higher food intake in a negative affective state versus in a neutral affective state. An interaction effect of emotional eating and induced mood on attentional biases and food intake was predicted. Second, we aimed to explore if maintained attention for food would be a better predictor of food intake in a negative affective state than selfreported emotional eating.

\section{Method}

\section{Participants}

Participants $(\mathrm{N}=85)$ were recruited through flyers, Facebook, and via e-mail. Only female students were eligible for participation, because emotional eating has been considered mainly prominent in women (van Strien, et al., 1986). Participants were randomly assigned to either the negative mood or the neutral mood condition.

\section{Materials and Procedure}

\section{Mood Induction}

A combination of music and autobiographical recall was used to induce a negative or a neutral mood, because similar mood inductions have been successfully used in previous research (Jansen et al., 2008; Martin, 1990). In the negative mood condition, participants listened to 'Adagio for Strings' by Samuel Barber for 8 minutes while writing about a recent negative experience, whereas participants in the neutral mood condition listened to a neutral piece of music 'Dancing with the Sun' by Celia Felix (2010) and wrote about a recent study course. A mood booster was 
introduced after the first half of the attention paradigm to ensure that mood levels remained negative/neutral during the testing procedure. During the booster, participants again listened to the respective music piece for 1 minute and had to reread their writings. Participants listened to the music pieces on headphones.

\section{Visual Probe Paradigm}

To assess attentional biases for food stimuli in contrast to neutral stimuli, eye movements and manual response latencies were recorded during a visual probe task. In this task two pictorial stimuli appear simultaneously on the left and right side of a computer screen, followed by a probe (in this case a small star) that replaces one of the stimuli. Participants are instructed to respond as quickly as possible to the location of the star by pressing a corresponding key on the key board. The logic of this task presumes that the attention of the participant is automatically drawn towards the personally more relevant stimulus, and therefore leads to faster responding to the star if the star replaces the image that draws the attention. Besides the assessment of response latencies to the probe location, attention processes were measured by the recording of eye movements that occur during the stimuli presentation. The same paradigm was already used in previous research (see Werthmann et al. 2011).

Overview. Each trial started with a fixation cross that disappeared directly after participants fixated on it, which was followed by the stimulus pair for 2000 ms. Then the probe appeared until participants made a manual response indicating the position of the probe.

The task consisted of 120 trials in total, which were split into two blocks of 60 trials each, during which a mood booster was introduced. In total, twenty critical stimulus pairs were presented four times, resulting in 80 critical trials. Additionally, ten non-relevant stimulus pairs were presented four times, resulting in 40 filler trials.

Stimuli. In critical trials, a photograph of a palatable high-caloric food stimulus was paired with a photograph of a non-food stimulus (music instrument). In filler trials, photographs of traffic objects were paired with photographs of office objects. All stimuli were digital photographs matched in colour, complexity and brightness. Food stimuli and non-food stimuli were equally distributed over the right and left side of the computer screen. The probe replaced equally often food and neutral stimuli and was equally distributed on the right and left screen location. The same stimulus-set was previously used by Werthmann et al. (2011).

Eye movements. Eye movements were recorded by a desktop mounted EyeLink 1000 system (SR Research Ltd., Mississauga, Ontario, Canada). A 9-point calibration with subsequent validation procedure was conducted prior to the visual probe paradigm. To assess attention allocation processes, participants' gaze fixations during stimuli presentations were studied. Gaze fixations were defined as any 
period that was not a blink or saccade and lasted at least $100 \mathrm{~ms}$ (Eyelink Dataviewer User's Manual, 2002-2008, SR Research Ltd.). Eye movements that occurred before the presentation of an image pair were excluded, because these movements could represent anticipatory fixations. For analysis purposes, the computer screen was, invisible for participants, divided into three areas of interest: the mid-section, indicating the location of the fixation cross, and the left and right section, representing the locations of the picture stimuli. Only eye movements in critical trials directed either to the left or the right section of the screen were extracted for further analyses. Eye movements in filler trials and gaze fixations in the mid area were excluded from further analyses. Eye movements were extracted using Data Viewer (SR Research Ltd., Mississauga, Ontario, Canada).

Visual attentional biases. On the basis of eye movement recordings, three visual attentional bias scores were calculated: (i) gaze direction bias which is defined as the proportion of trials on which the first fixation was directed to a food stimulus versus a non-food stimulus (a score above $50 \%$ indicates a higher proportion of first fixations on food); (ii) initial gaze duration bias, which is a measure for early attention maintenance, computed as the difference between food and neutral stimuli in the average duration of first fixations towards the initially fixated picture before the gaze was shifted away (a positive score indicates longer initial gaze on food than on non-food stimuli), and (iii) gaze dwell time bias, which is computed as the difference between average total dwell time on food and non-food stimuli (a positive score indicates longer dwell time on food stimuli than on non-food stimuli). For comparable measures, see for example Castellanos, et al., 2009; Mogg, et al., 2003; Werthmann, et al., 2011.

Response latency attentional bias. Participants' manual response latencies when reacting to the probe location were used to calculate a response latency bias. Following previous research (e.g. Castellanos, et al., 2009; Mogg, et al., 1998) response latencies that were faster than $200 \mathrm{~ms}$, slower than 2000, and then if they deviated more than 3 SDs from each participant's mean were excluded from further analyses. Bias scores were then calculated by subtracting the mean response latency on congruent trials (i.e. when the probe replaced a food image) from the mean response latency on incongruent trials (i.e. when the probe appeared in the same location as the preceding neutral image). A positive bias score is indicative for an attentional bias towards food stimuli, whereas a negative bias score can be interpreted as attentional avoidance, that is an attentional bias away from food stimuli.

\section{Food intake}

Food intake of participants was measured by a disguised ad libitum food offer. The experimenter disguised this measurement of food intake by telling the participant that she had to wait until the effect of the prior experimental manipulation (mood 
induction) wears off. The participant was further told that she should make herself comfortable, read a magazine and enjoy the offered food, if she wants to, and wait for about 10 minutes, after which the experimenter would come back and the rest of the experiment would continue. Both healthy (grapes and cucumber) and unhealthy (chocolate and chips) food items were offered. The bowls with food were placed on a table with magazines (about living decoration and travelling). The food bowls were weighted before and after the waiting time. The difference in weight indicated the amount of food consumption in grams and calorie intake was calculated subsequently. None of the participants who completed a subsequent awareness check ${ }^{6}$ were suspicious of this measure of food intake.

\section{Questionnaires}

Hunger, craving and mood. Throughout the experimental procedure visual analogue scales (VAS) were used to assess mood state, state craving, and hunger at five different time points (baseline (VAS1), post mood induction (VAS2), post mood booster (VAS3), post visual probe task (VAS4), post taste test (VAS5)). Participants had to indicate their mood on three VAS scales (asking for positive/negative affect, depressiveness/cheerfulness, and happiness/sadness), their feelings of hunger on two questions (asking for fullness and hunger) and their state craving on one VAS scale. All VAS scales were $100 \mathrm{~mm}$ horizontal lines running from 0 to 100 , where the opposing feelings (e.g. positive affect versus negative affect) were positioned. Higher scores indicated more positive mood/more hunger/more craving, lower scores denoted a more negative mood state/less hunger/less craving. For analysis purposes average mood ratings for each VAS scale were calculated by taking the mean rating of the three mood questions per time point. Similarly, one hunger score per time point was calculated by averaging ratings of the two VAS scales asking for feelings of hunger per time point. In addition, one open question asking participants to estimate the time passed since their last meal was included in the first assessment of mood, hunger and craving, as an additional check for satiety.

Dutch Eating Behavior Questionnaire (DEBQ). The DEBQ (van Strien, et al., 1986) measures eating behaviour characteristics on three subscales, assessing emotional eating (13 items), external eating (10 items) and restrained eating (10 items). Items are scored on a five-point Likert scale ranging from 0 (never) to 5 (very often) and higher scores indicate increased emotional eating, increased external eating and more restrained eating.

\footnotetext{
${ }^{6}$ Awareness checks were available for 64 participants.
} 


\section{Procedure}

All participants were tested individually by two experimenters. One experimenter conducted the first part of the procedure including the assessment of attention bias for food, then the other experimenter conducted the rest of the procedure. Upon arrival, the participant signed the informed consent and filled in the baseline measure of mood state, hunger and craving (VAS1). Then, the negative or the neutral mood induction followed, depending on whether the participant has been randomly assigned to the experimental or the control condition and the participant subsequently filled in VAS2. Afterwards she participated in the first half of the visual probe task, and then the mood induction was briefly repeated in a oneminute mood booster during a break between two blocks of the visual probe paradigm. Before starting the second block of the visual probe task, she filled in VAS3. After completion of the visual probe task, the participant filled in VAS4. Then, a cover story was used to disguise the direct assessment of food intake, which was measured after the participant was left alone with four kinds of food for 10 minutes. Upon return of the experimenter, the participant filled in VAS5 and then filled in the additional questionnaires (restraint scale by Herman \& Polivy, 1980, DEBQ, behavior approach/behavior inhibition scales (BIS/BAS) by Carver \& White, 1994). A brief awareness check was conducted afterwards. At the end of the experiment, weight and height were measured, the participant was thanked for her participation and was compensated with either a course credit or $€ 7,50$.

\section{Analyses}

Independent sample t-tests were conducted to test whether the experimental and the control group differed on hunger, craving, baseline affective state, restraint scores (as assessed with the restraint scale and the DEBQ), external eating scores, emotional eating scores, (as assessed with the DEBQ), impulsivity (as assessed with the BIS/BAS scales), age and body mass index (BMI) as randomization check. To check whether the mood induction was successful a repeated measures analyses of variance (ANOVA) with average mood ratings from the VAS scales on 5 time points as within-subjects variable and experimental condition (neutral, negative) as between-subjects variable was tested. Hierarchical multivariate linear regression analyses were conducted to test if self-reported emotional eating is related to attention to food and food intake in a negative affective state versus a neutral state (Hypothesis 1). For this aim, five linear hierarchical multivariate regression models (for direction bias, initial gaze duration bias, dwell time bias, response latency bias and for food intake in calories) were conducted to test the effects of emotional eating and affective state on attention and on (over)eating. In the first step emotional eating (centred) and the mood condition (as dummy variable) were entered. In the second step the emotional eating $\times \operatorname{mood}$ condition interaction was entered. 


\section{CHAPTER 6}

In line with the hypothesis a significant interaction effect of emotional eating and mood condition on increased attention and food intake was expected.

To test our second research question, if maintained attention (i.e., dwell time bias) would be a better predictor of food intake in a negative affective state than self-reported emotional eating, another hierarchical multivariate linear regression analysis was conducted. In the first step, mood condition (as dummy variable), emotional eating (centred variable) and dwell time attentional bias scores (centred variable) were entered. In the second step, the interactions of emotional eating $x$ mood condition and of dwell time attentional bias scores $\times$ mood condition were entered.

\section{Results}

\section{Group characteristics}

Independent sample $t$-tests confirmed that there were no significant differences between participants in the negative mood and the neutral mood condition with regard to self-reported hunger, craving, BMI, age, restraint scores, external eating scores, emotional eating scores, impulsivity, and affective state at baseline (all $t$ s < 1.24 and all $p s>.22$ ). Participants in the neutral mood condition, however, reported a significant longer time since their last meal than participants in the negative mood condition. Yet, self-reported hunger scores did not seem to be influenced by this difference. Thus, participants in both conditions arrived in similar mood and hunger states for the experiment. See Table 1 for all participant characteristics. 
Table 1

Main sample characteristics at baseline for participants in the neutral and negative experimental condition, respectively.

\begin{tabular}{|c|c|c|c|c|c|c|}
\hline & \multicolumn{2}{|c|}{$\begin{array}{l}\text { Neutral condition } \\
(\mathrm{n}=43)\end{array}$} & \multicolumn{2}{|c|}{$\begin{array}{l}\text { Negative condition } \\
(\mathrm{n}=42)\end{array}$} & \multirow[b]{2}{*}{$t(83)$} & \multirow[b]{2}{*}{$p$} \\
\hline & $M$ & $S D$ & $M$ & $S D$ & & \\
\hline Mood at baseline & 73.80 & 12.25 & 75.97 & 11.54 & 0.84 & .40 \\
\hline Hunger at baseline & 41.93 & 19.90 & 41.45 & 16.58 & 0.12 & .91 \\
\hline Craving at baseline & 38.49 & 25.44 & 36.00 & 21.30 & 0.49 & 63 \\
\hline Time since last meal (in min) ${ }^{7}$ & 103.64 & 64.00 & 68.22 & 57.26 & 2.66 & .01 \\
\hline RS score & 11.21 & 4.77 & 9.98 & 4.42 & 1.24 & .22 \\
\hline Emotional Eating (DEBQ) & 2.70 & 0.66 & 2.58 & 0.68 & 0.83 & .41 \\
\hline External Eating (DEBQ) & 3.47 & 0.53 & 3.30 & 0.44 & 1.52 & .13 \\
\hline Restrained Eating (DEBQ) & 2.70 & 0.77 & 2.57 & 0.79 & 0.82 & .42 \\
\hline Reward Drive (BAS) & 11.70 & 1.79 & 11.26 & 1.81 & 1.12 & .27 \\
\hline Fun Seeking (BAS) & 11.93 & 1.84 & 11.81 & 1.60 & 0.32 & .75 \\
\hline Reward Response (BAS) & 17.70 & 1.87 & 17.43 & 1.88 & 0.66 & .51 \\
\hline BIS & 21.98 & 2.98 & 21.62 & 3.57 & 0.50 & .62 \\
\hline BMI & 22.13 & 2.71 & 21.63 & 2.24 & 0.92 & .36 \\
\hline Age & 20.81 & 2.06 & 20.48 & 1.99 & 0.77 & .45 \\
\hline
\end{tabular}

Note. $\mathrm{RS}$ score $=$ Restraint Scale (Herman \& Polivy, 1980), DEBQ $=$ Dutch Eating Behavior Questionnaire (van Strien, et al., 1986), BMI = Body Mass Index, BIS/BAS = Behavior Activation/Behavior Inhibition Scales (Carver \& White, 1994).

\section{Mood induction}

Results from the repeated measures ANOVA testing whether the mood manipulation successfully induced a mood decrease in the negative mood condition showed a significant mood condition $\times$ time interaction, $F(2.74,82)^{8}=38.79, p<.001$, suggesting that mood indeed decreased significantly in the negative mood condition but remained unaltered in the neutral mood condition. Independent-samples $t$ tests confirmed significant differences between conditions at time 2 , thus after the induction, $\left(t(82)^{9}=6.19, p<.001\right)$, time 3 , that is after the mood booster, $(t(83)=$

\footnotetext{
${ }^{7}$ Two participants did not fill in this question and were counted as missing for this analysis. The power of the $t$-test is based on 81 degrees of freedom.

${ }^{8}$ Mauchly's test indicated that the assumption of sphericity had been violated, therefore degrees of freedom were corrected using Greenhouse-Geisser estimate of sphericity.

${ }^{9}$ One participant did not fill in the VAS at time 2
} 
$5.91, p<.001)$, and time 4 , prior to the taste test, $(t(83)=3.48, p<.01)$. At time 5 (at the end of the experiment; that is after a 10 minutes break) no significant difference between conditions was detected $(t(83)=1.23, p=.22)$. See Figure 1 for changes in mood per condition for all time points.

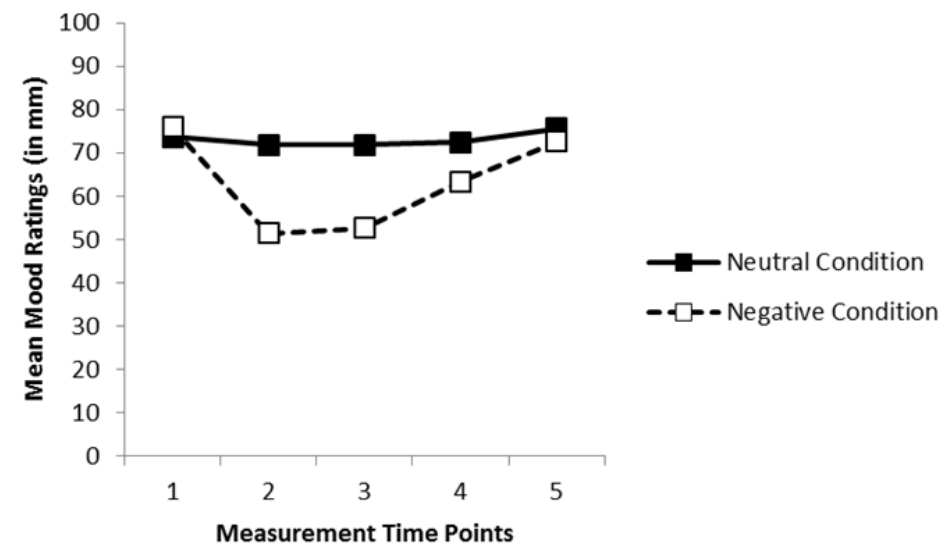

Figure 1. Mean mood ratings (lower values indicate stronger negative affect) per condition (negative versus neutral condition) on time 1 (at baseline), time 2 (after the mood induction), time 3 (mood booster during visual probe task), time 4 (prior to taste test) and time 5 (after food intake measure).

\section{Main Analyses}

Effect of Condition and Emotional Eating on Attention for Food. Results from the regression analyses on the influence of emotional eating and affective state on attention for food revealed no significant main effect of emotional eating, all $\beta \mathrm{s}<$ .17 , ts $<1.52$, ps $>.13$. There was a significant main effect of the mood condition on the direction of attention, $\beta=.22, \mathrm{t}=2.02, \mathrm{p}=.047$, indicating that participants in the neutral mood condition had a significantly higher initial orientation towards food $(\mathrm{M}=55.38, \mathrm{SD}=7.53)$ than participants in the negative mood condition $(\mathrm{M}=$ $52.74, \mathrm{SD}=4.56)$. No significant emotional eating $\times \operatorname{mood}$ condition interaction effects were observed on any attentional bias scores, all $\beta \mathrm{s}<.09$, all ts $<0.58$, all ps $>$.56. The inclusion of the interaction term of emotional eating $\times$ mood condition did not add to explained variance of the model, all $\mathrm{Rs}^{2}<.06$, all $\Delta \mathrm{R}^{2} \mathrm{~s}<.004$, all $\mathrm{Fs}(3,81)<1.68$ all ps $>.56$. Results from these regression analyses thus indicate that self-reported emotional eating was not associated with attention for food during negative mood. 
Effect of Condition and Emotional Eating on Food Intake. No significant main effect of emotional eating, $\beta=.11, t=0.95, p=.34$ and no significant emotional eating $\times$ mood condition interaction effect, $\beta=.12, t=0.74, p=.46$, were observed for food intake ${ }^{10}$. The inclusion of the emotional eating $\times$ mood condition interaction did not add explained variance to the model, $\mathrm{R}^{2}=.02, \Delta \mathrm{R}=.004, F(3,81)=0.51, p=$ .68. This means that self-reported emotional eating was not associated with food intake during negative affective state.

Association of maintained attention on food intake in a negative mood. As no significant relation between emotional eating and food intake was found in the previous analysis, we decided to conduct the final regression analysis only on the effect of maintained attention and mood condition on food intake. The results of this regression analysis showed in step 1 a significant main effect of attentional maintenance (i.e. dwell time bias scores) on food intake: longer dwell time scores were associated with more food intake, $\beta=.35, t=3.35, p<.01$. More importantly the interaction term of dwell time attentional bias scores $\times$ mood condition was significant in step two of the analysis, $\beta=-.32, t=2.38, p<.05$, see Table 2 for all respective coefficient values.

Table 2

Summary of linear hierarchical multivariate regression analysis of variables predicting food intake (in cal.)

\begin{tabular}{llllll}
\hline Step & Predictor & B (SE) & $\beta$ & t & $p$ \\
\hline Step 1 & Mood condition & $-1.63(24.45)$ & -.007 & -0.07 & .947 \\
& Dwell Time & $0.20(0.06)$ & .347 & 3.35 & .001 \\
Step 2 & Mood Condition x Dwell Time & $-0.29(0.12)$ & -.319 & 2.38 & .020 \\
\hline
\end{tabular}

Note. $R^{2}=.12$ for Step $1 p=0.005 ; R^{2}=.18, \Delta \mathrm{R}^{2}=.06$ for Step $2, p=0.02$

To further explore the direction of this significant interaction, simple slope testing was conducted. Simple slopes testing the effect of dwell time attentional bias scores per condition showed that dwell time on food affected food intake significantly in the neutral mood condition; $\beta=.56, t=4.16, p<.001$, longer attentional maintenance was associated with more food consumption, yet, in the negative mood condition dwell time for food did not affect food intake; $\beta=-.35, t=0.47, p=$ .642 (see Figure 2). Simple slope testing for the effect of high (+ 1 SD) or low ( 1 SD) dwell time for food on subsequent food intake showed a trend for differences between conditions: participants with longer dwell time for food (+ 1 SD) ate trend-significantly more food in the neutral condition than in the negative condi-

${ }^{10}$ As food intake was positively skewed, sensitivity analyses with log-transformed food intake were conducted. Results remained the same, and results here reported are based on the original values of food intake (in calories). 
tion; $\beta=-.25, t=-1.75, p=.084$. Participants with shorter dwell time for food $(-1$ SD) did not differ in their food intake between conditions; $\beta=.24, t=-1.64, p=.11$. These results mean that longer attentional maintenance on food stimuli was significantly associated with higher food intake in the neutral but not in the negative mood condition, whereas self-reported emotional eating was not predictive of food intake, in neither condition.

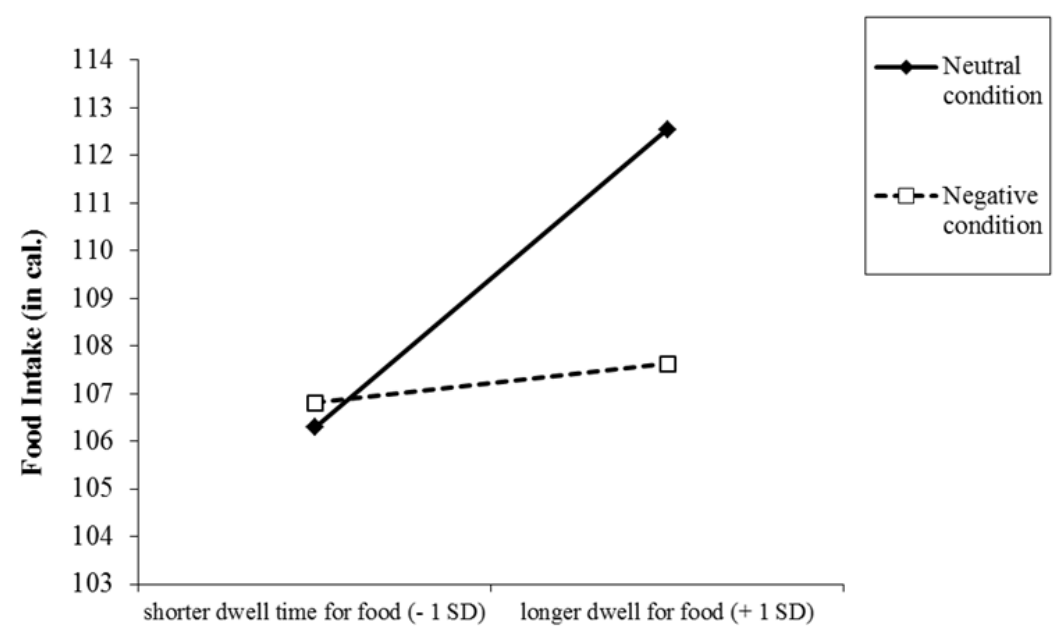

Figure 2. Food intake (in cal.) as a function of dwell time attentional bias scores (1 SD below and 1 SD above the mean dwell time attentional bias score, respectively) and mood condition (negative and neutral mood induction).

\section{Discussion}

The main aim of this study was to test experimentally if self-reported emotional eating is related to increased attention bias for food and increased food intake after a sad mood induction. A second aim was to test whether the attention maintenance bias component, that is dwell time on food stimuli, predicts food intake during sad mood to a larger extent than self-reported emotional eating (based on the DEBQ).

Our results indicate that self-reported emotional eating, at least when assessed by the DEBQ, did neither account for attentional biases for food nor for actual food intake during sad mood, in the current sample. With regard to food intake, our results dovetail with previous findings that also did not find elevated food intake in response to negative feelings in self-identified emotional eaters according to the DEBQ (e.g. Adriaanse, et al., 2011; Bongers, et al., 2013; Bongers, Jansen, Houben, \& Roefs; Conner, et al., 1999; Evers, et al., 2009; Evers, de Ridder, 
\& Adriaanse, 2010). Other authors have already offered an explanation for this observation: for example Adriaanse and her colleagues (2011) suggested that selfassessment of emotional eating might be difficult (and therefore invalid) because of a possible recall bias as emotional states can affect recall immensely.

With regard to attention bias for food, the current results demonstrate that self-reported emotional eating was not related to increased attention for food during negative mood in this student sample. The current study is the first evidence, to our knowledge, that tested this relation directly by means of eye-tracking. In line with our finding, one previous study using a response latency-based measure for attentional processes did also not find an association of emotional eating and a response latency-based attentional bias for food, thereby also suggesting that selfreported emotional eating is not related to increased attention bias for food during sad mood (Hepworth, et al., 2010). Altogether, the current results contribute to further evidence that self-reports of emotional eating, at least as measured by the DEBQ, do not accurately predict increased attention bias for food or increased food intake when feeling sad (Adriaanse, et al., 2011; Bongers, et al., 2013; Bongers, et al.; Evers, et al., 2009; Evers, de Ridder, et al., 2010; Hepworth, et al., 2010).

Whereas our results concerning the link of emotional eating and attention for food and food intake are similar to an earlier study, our findings differ from the results by Hepworth and her colleagues (2010) with regard to changes in attention due to an experimental mood induction. Hepworth et al. (2010) observed a main effect of their mood induction on their measure of attention bias for food, in that a negative mood increased attention bias for food cues, whereas results from our study showed that the negative mood induction decreased initial orientation towards food. These differences are not likely due to different mood induction procedures, because fairly similar experimental procedures were applied in both studies. However, the divergence of findings might be explained by a different choice in the assessment of attention bias for food: Hepworth and her colleagues used a response latency-based, thus indirect, measure of visual attention whereas in the current study a more direct assessment of visual attention, namely eye-movement recordings were included. In addition, Hepworth et al. collapsed the two attentional components (an early, assessed at $500 \mathrm{~ms}$, and a later component, assessed at $2000 \mathrm{~ms}$ ) when reporting their main effect of mood induction on attention measures, whereas we analyzed the data of the four attention components assessed in our study separately.

Even though an interpretation remains speculative at this point and needs further testing, our finding that negative affect decreases an orientation bias for food might indicate that negative mood in general decreases the attractiveness and/or rewarding value of otherwise highly salient and rewarding stimuli. This suggestion fits with recent evidence showing that sad mood decreases reward sensitivity (Foti \& Hajcak, 2010; Hervas \& Vazquez, 2013). Another explanation for 
the finding that initial orientation bias to food cues was higher in the neutral condition, than in the negative mood condition, is that more time had elapsed since the last meal for participants in the neutral condition. However, this explanation is unlikely because the mean hunger ratings did not differ between the two groups ( $41 \mathrm{~mm}$ on a $100 \mathrm{~mm}$ scale ranging from not hungry (0) to very hungry (100) for both groups).

With regard to our second hypothesis, our results demonstrated that longer dwell time on food stimuli was associated with increased food intake, at least in the neutral condition, within the current sample of female students. This finding suggests that attentional maintenance on food is related to subsequent food intake, because individuals with longer attention focus on food were inclined to consume more food. Apparently maintaining one's gaze on food contributes to food intake, in a neutral affective state. In this respect, this finding corroborates with previous suggestions that attention focus on food is related to food consumption (Hofmann \& Van Dillen, 2012; Kavanagh, Andrade, \& May, 2005; Tiggemann \& Kemps, 2005; J. Werthmann, Field, Roefs, Nederkoorn, \& Jansen, 2013; Jessica Werthmann, et al., 2013). Yet, against our hypothesis, during negative mood this relation could not be established.

Altogether, our current results suggest that attentional biases for food could eventually constitute a cognitive mechanisms contributing to overeating, even though not during negative mood. Attention biases should therefore be assessed as additional and informative measure next to self-reported eating motivation. Selfreported eating motivation might be inaccurate due to social desirable response tendencies. For example, it has been shown that participants who score high on a social desirability questionnaire also report lower emotional eating scores on the DEBQ (Allison \& Heshka, 1993). Assessment of eating motivation using behavioral or physiological assessments might therefore compliment self-report instruments in situations when social desirable response tendencies are likely, for example when assessing eating motivation in overweight or underweight participants. As attentional biases for food can be directly linked to food intake, another advantage of this assessment is that attention bias might provide a more automatic, thus less obtrusive measurement of eating motivation, besides measure(s) of self-report.

In terms of clinical relevance our findings highlight the impact of an obesogenic food environment on eating behavior because they provide evidence that if attention is maintained on food cues, the chance of overeating increases. Yet, this interpretation should be viewed under some limitations of our study. We have tested female college students and therefore our results are not generalizable to other populations. In this respect, it would be fruitful for future research to explore if our findings can be replicated in clinical samples, for example in eating disordered patients, obese participants or depressed patients. Moreover, we feel inclined to mention that even though our mood induction produced a significant 
decrease in mood, the ratings of participants in the experimental group indicate that their mood was moderately negative instead of severely negative (mean mood rating was 51.37 on a $100 \mathrm{~mm}$ scale after the negative mood induction), or simply less positive than in the control group (mean mood rating was 71.89 after the neutral mood induction). Therefore, future research should determine whether negative affect and sad mood, in contrast to moderately negative mood have more pronounced effects on attention for food and food intake. Otherwise, it would also be interesting to explore whether positive mood affects attention for food and food intake.

Keeping these limitations in mind, the current research showed that individuals' attention focus for food is related to subsequent food intake and thereby suggesting that attention bias for food could be a cognitive mechanism contributing to overeating. However, during negative mood attention bias for food did not affected increased food intake. The extent to which this relationship is affected by more extreme mood changes remains to be explored. 



\section{Chapter 7}

\section{Attention bias for chocolate increases chocolate consumption: \\ An attention bias modification study}

Published as:

Werthmann, J., Field, M., Roefs, A., Nederkoorn, C., \& Jansen, A. (2014). Attention bias for chocolate increases chocolate consumption - An attention bias modification study. Journal of Behavior Therapy and Experimental Psychiatry, 45, 136-143 


\section{Abstract}

Objective: The current study examined experimentally whether a manipulated attention bias for food cues increases craving, chocolate intake and motivation to search for hidden chocolates.

Method: To test the effect of attention for food on subsequent chocolate intake, attention for chocolate was experimentally modified by instructing participants to look at chocolate stimuli ("attend chocolate" group) or at non-food stimuli ("attend shoes" group) during a novel attention bias modification task (antisaccade task). Chocolate consumption, changes in craving and search time for hidden chocolates were assessed. Eye-movement recordings were used to monitor the accuracy during the experimental attention modification task as possible moderator of effects. Regression analyses were conducted to test the effect of attention modification and modification accuracy on chocolate intake, craving and motivation to search for hidden chocolates.

Results: Results showed that participants with higher accuracy $(+1 S D)$, ate more chocolate when they had to attend to chocolate and ate less chocolate when they had to attend to non-food stimuli. In contrast, for participants with lower accuracy $(-1 S D)$, the results were exactly reversed. No effects of the experimental attention modification on craving or search time for hidden chocolates were found.

Limitation: We used chocolate as food stimuli so it remains unclear how our findings generalize to other types of food.

Conclusion: These findings demonstrate further evidence for a link between attention for food and food intake, and provide an indication about the direction of this relationship.

Keywords: Attention Bias, Attention Modification, Chocolate, Antisaccade Task, Eye-Tracking, Overeating 
In an obesogenic food environment, as present in the western world, we are constantly surrounded by an abundance of palatable food cues. Ignoring or attending to these food cues may influence our eating behaviour. For example, imagine you are walking through the main shopping street of your town on a Saturday: If you notice all the delicious food cues from shops or restaurants you may end up consuming a lot of high -calorie food, such as waffles, French fries and hamburgers. If you focus instead on shoes in shop windows, ignoring all food cues, you might end up with a full shoe cabinet, but probably not with a full stomach.

Attention for delicious, yet unhealthy, food temptations could contribute to a lack of control over food intake, for example by facilitating overeating or food intake of unhealthy snacks. Indeed, evidence from several studies measuring attentional bias for food suggests that attention for food is related to obesity and craving for food. For example, neuroimaging studies showed that a greater activation of reward and attention centres in the brain during food exposure is related to obesity, poorer weight control and weight gain (Murdaugh, Cox, Cook, \& Weller, 2012; Stice, Yokum, Bohon, Marti, \& Smolen, 2010; Yokum, Ng, \& Stice, 2011). In addition, elevated attention biases for food cues have been observed in overweight and obese samples in comparison to participants with a healthy weight (e.g., Castellanos et al., 2009; Nijs, Franken, \& Muris, 2010; Werthmann et al., 2011). Moreover, several studies showed that attention biases for food are related to hunger and craving in healthy weight and overweight participants (e.g., Kemps \& Tiggemann, 2009; Mogg, Bradley, Hyare, \& Lee, 1998; Nijs, Muris, Euser, \& Franken, 2010; Piech, Pastorino, \& Zald, 2010; Smeets, Roefs, \& Jansen, 2009; Werthmann, et al., 2011). Thus, evidence from these cross-sectional studies establishes an association of attention for food and eating-related behaviors or increased BMI but did not test the hypothesized causal relation of attention bias and food intake.

There is preliminary evidence suggesting that changes in attention for food cues may be causally related to changes in eating behaviour: one previous study that manipulated attention focus for food after a body image challenge (i.e., confronting participants with advertisements of thin models and body parts) showed that attending high-calorie food words elevated the chance to choose low-calorie over high- calorie cookies, in comparison to participants who attended neutral words (Smith \& Rieger, 2009). This finding suggests that attending to high-calorie foods and being exposed to body image comparisons could activate dietary restraint. However, because attention to food was manipulated in the context of body image satisfaction and because the amount of cookies was fixed, this study does not inform about the relation of purely the attention for food and the amount of food intake.

Thus, given the correlational nature of most previous studies, the causal impact of attention for food cues on food intake remains unclear, and this question 
can only be addressed by experimentally manipulating attentional bias followed by a measure of food intake. The aim of the current study was therefore to manipulate attention bias for food cues, to test whether a causal relationship exists between manipulated attention for food on the one hand and craving and food intake on the other hand.

Experimentally inducing changes in attentional processes to affect subsequent behaviour has been termed attention bias modification (ABM). This method has been explored extensively in the field of anxiety research. Several studies have shown that training attention away from threatening stimuli reduced an attentional bias for these cues and led to a decrease in anxiety symptoms in adults and children in comparison to a control group (Bar-Haim, Morag, \& Glickman, 2011; Hakamata et al., 2010; Hallion \& Ruscio, 2011; Mathews \& MacLeod, 2002).

Typically, ABM studies have relied on a modified version of the visual-probe paradigm as training. During this task, two stimuli (symptom-relevant/neutral) are presented side by side on a computer screen, and then a probe appears in the location of one of the stimuli. Participants are instructed to react to the probe by a manual response. To manipulate attention towards (or away) from disorderrelated cues, the probe replaces the relevant stimuli in $100 \%$ (or $0 \%$ ) of all trials. The rationale of the training presumes that an implicit learning process is elicited through attending to the systematic contingencies, which improves task performance, thereby gradually modifying an attention bias towards (or away from) the disorder-relevant cue (Bar-Haim, 2010). However, one disadvantage of modifying the visual probe paradigm in this way is that it modifies attention processes indirectly by manipulating and measuring the manual response reactions. Yet, targeting visual attention processes directly by the modification procedure and simultaneously monitoring visual attention as measure for modification accuracy, for example by recording eye movements, could provide a more precise modification of attention.

One alternative approach to manipulate attention biases, which overcomes the disadvantage of modifying attention indirectly, is a modified antisaccade task in combination with the assessment of eye-movements. Saccades are rapid eyemovements (e.g., Munoz \& Everling, 2004). Saccadic eye-movements are closely related to visuo-spatial attentional engagement and represent a faster measure for attentional processing than the recordings of response reactions (e.g., Bannerman, Milders, de Gelder, \& Sahraie, 2009; Hutton, 2008; Munoz \& Everling, 2004). The antisaccade task has typically been used as a measure of cognitive inhibitory control (e.g., Hallett, 1978; Hallett \& Adams, 1980; or for a review Hutton, 2008). In general, the antisaccade task can be seen as a stimulus-response mapping task with regard to attentional processing: During the task participants are required to inhibit reflexive eye movements (saccades) towards a peripherally presented target, and have to shift their gaze in the opposite direction (i.e. perform an antisac- 
cade). This process requires a top-down inhibitory cognitive control execution, because participants have to suppress their reflexive response (prosaccade towards target), and instead have to voluntarily initiate the inverse response by saccading towards the mirror position of the target (Munoz \& Everling, 2004). Thus, this method provides the possibility to combine a direct modification of attention with the monitoring of accuracy by implementing recordings of eyemovements.

In the present study, the standard antisaccade task was adapted to manipulate attention towards versus away from chocolate. The aim of the current study was to test experimentally if modification of attention for chocolate versus attention for shoes would contribute to differences in chocolate consumption, changes in craving or motivation to search for hidden chocolates (as indexed by search time). To modify attention allocation for chocolate vs. neutral cues the contingencies of proand antisaccades towards or away from chocolate were altered: In the "attend chocolate" group, participants always had to perform a prosaccade towards chocolate stimuli and an antisaccade away from neutral stimuli (shoes), whereas in the "attend shoes" condition, participants always had to perform a prosaccade towards shoes and an antisaccade away from chocolate. We expected that participants in the "attend chocolate" group would consume more chocolate, report more craving and search longer for hidden chocolate, as index for higher motivation for chocolate, in comparison to participants in the "attend shoes" group.

Another advantage was that eye-movements were monitored during the antisaccade task. This is the first study that applied a measure for the accuracy of attention during an attention modification procedure. Thus, we further tested if accuracy during the attention modification moderated the influence of modified attention on chocolate consumption, craving and search time for hidden chocolates.

\section{Method}

\section{Participants}

Female participants $(n=56)$ were recruited by flyers and the local electronic recruitment system. Only female students were eligible for participation to eliminate possible gender effects with regard to eating behaviour. All participants were undergraduate students. The study received ethical approval from the local ethics committee. 


\section{Materials and Measures}

\section{Experimental Attention Modification: Antisaccade task}

Attentional bias for food cues was manipulated by a modified version of an antisaccade task (e.g., Hallett, 1978). Saccadic eye movements were recorded during the task to check how accurately the participant followed the attention modification instructions (i.e., modification accuracy). The paradigm was programmed with Experiment Builder (SR Research Ltd., Mississauga, Ontario, Canada).

Each trial started with a black fixation point appearing in the middle of the screen, which dissolved directly after participants fixated on it for $500 \mathrm{~ms}$. It was immediately replaced by either a red or blue fixation point which was presented in the central position for $1000 \mathrm{~ms}$, followed by a $200 \mathrm{~ms}$ gap (blank screen), which helps to speed up reaction times (e.g., Kissler \& Keil, 2008). The colour of this second fixation point informed participants whether to subsequently perform either a pro- (blue colour) or an antisaccade (red colour) to a pictorial stimulus (chocolate or shoe (neutral) pictures) that then appeared on either the left or right of the computer screen for 500 ms, see Figure 1 for a graphical presentation.

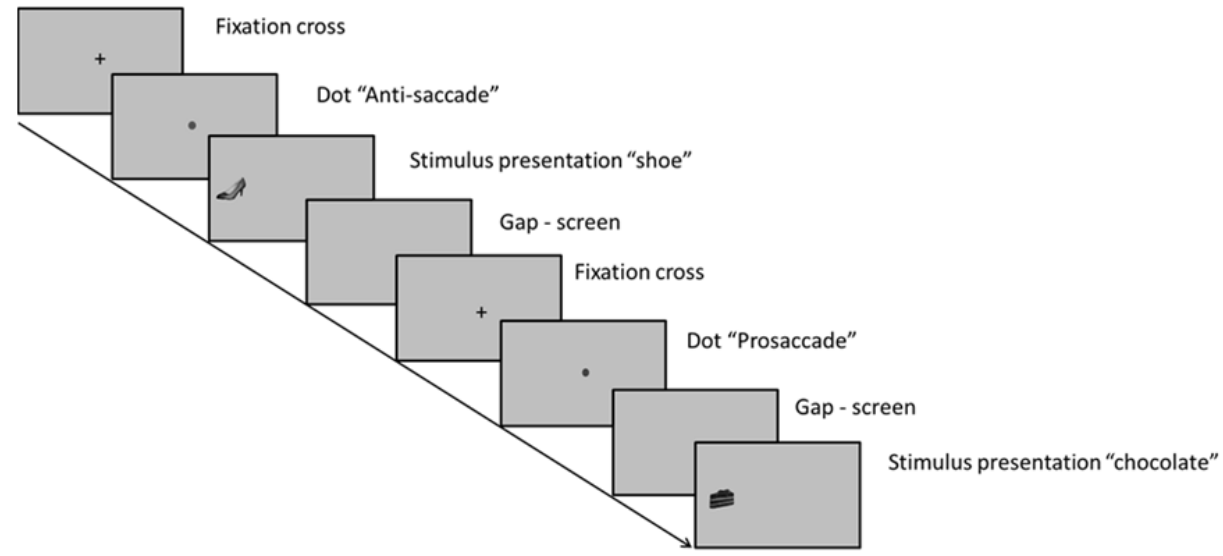

Figure 1. Schematic presentation of trials during the modification task. Depending on the respective condition, participants had always to make a prosaccade towards chocolate and an anti-saccade away from shoes during the stimulus presentation (in the "attend chocolate condition", as depicted here) or they had always to make an anti-saccade away from chocolate and a pro-saccade towards shoes (in the "attend shoe" condition, not depicted).

Trial Types. In total, each participant performed 320 trials, which were preceded by 40 practice trials. In the practice block, pro- and anti-saccade cues appeared before chocolate and shoe pictures equally often, that is, there were 10 chocolate prosaccade trials, 10 shoe prosaccade trials, 10 chocolate antisaccade trials and 10 shoe antisaccade trials. The practice block was followed by eight attention modification blocks, each containing 40 modification trials. In each block 
20 chocolate pictures and 20 shoe pictures were presented. In the 'attend chocolate' condition, chocolate pictures were always presented after the prosaccade cue and shoe pictures were always presented after the antisaccade cue. These contingencies were reversed in the 'attend shoe' condition. The order of pro- and antisaccades trials was uniquely randomized for each participant within each block.

Stimuli. Chocolate stimuli consisted of 20 photographs of chocolate items. Shoe stimuli consisted of 20 photographs of shoes. Chocolate was contrasted with shoe stimuli, for two reasons: first, chocolate is regarded as palatable, yet forbidden food item and one of the food items particularly craved by women (Hetherington \& Macdiarmid, 1993). We wanted to contrast chocolate with a nonfood object that might in general be equally attractive for women, and decided therefore for shoes. Another reason for shoes as contrast category for chocolate stimuli was that both items were easy to match on colour. Accordingly, all stimuli were matched as closely as possible based on colour, complexity, brightness, object form and image size. The image size and position of stimuli were tested in a brief pilot study to ensure that participants in both conditions were able to recognize peripherally a stimulus as a chocolate or shoe photograph even if they had to perform an anti-saccade. Shoe photographs subtended at $5.33^{\circ} \times 5.12^{\circ}$, chocolate

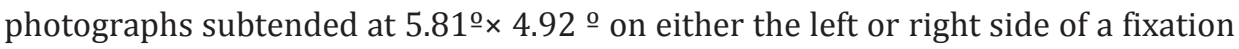
circle (subtending at $0.95^{\circ} \times 0.95^{\circ}$ ), at a viewing distance of $50 \mathrm{~cm}$. The position of chocolate and shoe cues (left or right of the centre) was fully counterbalanced within each block. All stimuli were presented against a light grey background (RGB colour spectrum: 191, 191, 191). All stimuli were presented on a Dell Optiplex ${ }^{\text {TM }}$ 760 Computer with a 19 inch monitor (60 Hz refresh rate) in a dimly lit room.

Eye movements. Eye-movements were monitored to measure accuracy during the attention modification task. The rationale for including this assessment of accuracy was to test if accuracy during the modification moderates the impact of attention modification on subsequent chocolate intake, craving and search time for hidden chocolates. In addition, eye-movement data was used to test if the modification of attention led to the expected differences in attention for chocolate. Therefore, two attention variables were computed based on eye-movement recordings: (1) the accuracy of performance was calculated based on the overall percentage of correctly performed trials, comprising correct performance on both pro- and antisaccade trials during the attention modification. (2) dwell time on chocolate stimuli during chocolate trials was calculated by averaging fixation durations on chocolate images per trial over all chocolate trials, irrespective of correctly or incorrectly performed, and then calculated as percentage score of average total fixation time per trial. Dwell time provides a measure for attentional focus on chocolate images (see for a comparable measure e.g. Castellanos et al. (2009)). 
Search Task

A search for hidden chocolates was included as a new method to assess motivation for chocolate. Persistence in task performance is a common measure for motivation (Keatley, Clarke, \& Hagger, 2012; Miller \& Hom, 1990) and was thus included to assess the participants' motivation for chocolate. For this search task the experimenter left the room and the participant received all further instructions via the computer screen. She was informed that two pieces of chocolates were hidden in the laboratory room and that her task was to search for them and find them as quickly as possible. She was further instructed to start and end the task by pressing a corresponding key on the computer keyboard. What the participant did not know was that actually only one piece of chocolate was hidden. The participant could abort searching for the second piece of chocolate by herself (by pressing the assigned computer key) or the search task was automatically aborted by a time-out after $7 \mathrm{~min}$. The time passed until the participant quitted searching for the second piece by herself or automatically after $7 \mathrm{~min}$, whatever came first, was recorded as a measure of motivation to search for chocolate. Search time was recorded in ms and transformed to minutes for further analysis.

\section{Chocolate Intake}

At the end of the search, the experimenter feigned surprise about having forgotten to hide a second piece of chocolate and offered, as excuse, a chocolate gift, which was that the participant could take as many chocolate pieces as she wanted from a large bowl filled with chocolate pieces. The test bowl was weighed before and after the participant had taken chocolate(s), to provide a measure of chocolate intake in grams.

\section{Questionnaires}

The participant reported her subjective hunger and craving on $100 \mathrm{~mm}$ visual analogue scales (VAS), which were "hidden" among three irrelevant questions about concentration and mood. To disguise the aim of our study and to avoid suspicion by participants prior to the attention modification task, the craving and hunger questions did not explicitly ask for craving (or hunger) for chocolate specifically, but for craving and hunger in general. At the end of the experiment, the participant also completed the Restraint Scale (RS; Herman \& Polivy, 1980) as a measure of weight and dieting concerns, the Dutch Eating Behaviour Questionnaire (DEBQ; van Strien, Frijters, Bergers, \& Defares, 1986), as a measure of external eating, emotional eating and restrained eating, and the Attitudes to Chocolate Questionnaire (ACQ; Benton, Greenfield, \& Morgan, 1998), as a measure of chocolate eating and problematic chocolate craving. 
Body Mass Index. The body mass index (BMI; $\mathrm{kg} / \mathrm{m}^{2}$ ) was calculated by measuring the weight and height of each participant at the end of the experiment.

\section{Procedure}

Prior to testing, participants received a reminder e-mail in which they were asked to refrain from eating for two hours before coming to the laboratory. All participants were tested between 11 am and $5 \mathrm{pm}$. Participants were randomly allocated to one of the two experimental groups and all were tested individually in a dimly lit laboratory room. All participants provided informed consent before taking part in the study. Upon arrival, the participant filled in the baseline measure of craving and hunger. Then her dominant eye was determined and after a 9-point calibration and validation procedure, she started with the practice task and proceeded with her respective attention modification condition. The antisaccade task took approximately $35 \mathrm{~min}$ to complete. Immediately after the attention modification task, craving and hunger were assessed again. Then the bogus chocolate-search test was conducted and chocolate intake was assessed. Subsequently, all participants were asked to write down what they thought the purpose of this study was (to probe for suspicion) before filling in the Restraint Scale, DEBQ and the ACQ, before her height and weight were measured. The participant was thanked for her participation and compensated with a course credit or 7.50 Euro gift voucher.

\section{Data Reduction}

Eye movements were recorded with an EyeLink 1000 system (SR Research Ltd., Mississauga, Ontario, Canada), with a temporal resolution of $1.4 \mathrm{~ms}$ and a spatial resolution of $0.01^{\circ}$. During data acquisition the eye tracker was automatically recalibrated whenever the fixation fell outside $3.64^{\circ} \times 3.40^{\circ}$ of the fixation point. For data analysis purposes, trials were discarded when saccades were undershoots (i.e., saccades with an amplitude $<2^{\circ}$, see Nummenaan, 2010), or anticipatory saccades (i.e., saccades that occurred less than $80 \mathrm{~ms}$ after target presentation, see Bannermann 2010; Taylor \& Hutton, 2009). Based on previous research (e.g., Unsworth, Schrock, \& Engle, 2004; Wieser, Pauli, \& Muhlberger, 2009), primary saccades were analysed, and the first saccade in each trial was categorized as an error or as a correct saccade based on its endpoint. For this purpose the computer screen was (hidden from participants), sub-divided into three basic areas: a midsection (subtending at $7.06^{\circ} \times 8.66^{\circ}$ ), in which the fixation point was presented, the far left and the far right side of the screen (subtending at $33.62^{\circ} \times 10.26^{\circ}$, respectively), representing the regions of the screen surrounding the target (on prosaccadic trials) or the mirror position of the target (on anti-saccadic trials). Based 
on these inclusion criteria, on average approximately $1.1 \%$ of data $(S D=1.48)$ was omitted per participant.

Data from five participants were removed completely because of recording failure during practice $(n=1)$ or because they had outlying high rates of missing data $(>3 S D)$ during the practice trials $(n=3)$ or during the attention modification task $(n=1)$. Data of the remaining 51 participants were included in the analysis ${ }^{11}$.

\section{Results}

\section{Differences at baseline}

There were no significant differences between experimental groups on any eating behaviour characteristics, such as restrained eating, chronic chocolate craving or BMI, or on their craving or hunger reports at baseline. Yet, there was a trend for participants in the "attend chocolate" group to express higher levels of external eating ${ }^{12}$. Descriptive statistics are shown in Table 1.

Table 1.

Main sample characteristics for participants in the "attend shoes" and in the "attend chocolate" training conditions, respectively.

\begin{tabular}{|c|c|c|c|c|c|c|}
\hline & \multicolumn{2}{|c|}{$\begin{array}{l}\text { "Attend shoes" } \\
(\mathrm{n}=26)\end{array}$} & \multicolumn{2}{|c|}{$\begin{array}{l}\text { "Attend chocolate" } \\
(\mathrm{n}=25)\end{array}$} & \multirow[b]{2}{*}{$t(49)$} & \multirow[b]{2}{*}{$p$} \\
\hline & $M$ & $S D$ & $M$ & $S D$ & & \\
\hline Age & 19.50 & 1.82 & 19.28 & 1.93 & 0.42 & .68 \\
\hline BMI & 22.01 & 3.60 & 22.24 & 2.53 & 0.27 & .79 \\
\hline Craving at baseline & 50.58 & 29.46 & 48.28 & 24.98 & 0.30 & .77 \\
\hline Hunger at baseline & 51.42 & 28.78 & 44.20 & 26.06 & 0.94 & .35 \\
\hline RS score & 12.85 & 6.40 & 12.52 & 6.16 & 0.19 & .85 \\
\hline Chocolate craving (ACQ) & 31.39 & 14.31 & 34.16 & 20.89 & $0.54^{+}$ & .59 \\
\hline Restrained eating (DEBQ) & 3.02 & 0.96 & 2.93 & 0.73 & $0.39^{+}$ & .70 \\
\hline Emotional eating (DEBQ) & 2.40 & 0.74 & 2.51 & 0.54 & $0.59^{*}$ & .56 \\
\hline External eating(DEBQ) & 3.10 & 0.63 & 3.43 & 0.56 & $1.97^{*}$ & .054 \\
\hline
\end{tabular}

Note. Chocolate craving ACQ = Chocolate craving subscale of the Attitudes to Chocolate Questionnaire (Benton, et al., 1998), DEBQ = Dutch Eating Behavior Questionnaire (van Strien, et al., 1986).

+ based on $d f=47$, because two participants did not fill in all questions.

* based on $d f=48$, because one participant did not fill in all questions.

\footnotetext{
11 We conducted a sensitivity analysis that included these outliers and results remained the same.

12 The main analyses were repeated with external eating (centred) as a covariate. Results remained the same. The reported analyses do not include external eating as a covariate.
} 
Eye-tracking was integrated in this study with the aim to monitor how accurately participants followed their respective modification instructions, as possible moderator of effects. Overall, participants made correct saccades $82.32 \%(S D=7.59)$ of trials during the attention modification task. Independent samples $t$-tests further confirmed that accuracy did not differ between experimental groups, $t(49)=0.92$, $p=.36$.

To check for the possibility that underlying participants' characteristics affected the ability to comply with the modification instructions (i.e. accuracy), additional correlation analyses were performed. Accuracy was not related to individual characteristics such as BMI, eating characteristics (restrained eating, the three DEBQ measures of eating behaviour, trait chocolate craving), craving or hunger, all $r s<-.22$, all $p s>.11$.

Another aim of the eye-tracking was to test if the attention modification was successful in producing differences in attention for chocolate. For this aim the dwell time on chocolate was calculated and compared between conditions by means of an independent sample t-test. Overall, participants differed in their dwell time on chocolate stimuli during chocolate trials, in their respective condition, $t(51)=43.42, p<.001$. Participants in the attend-chocolate condition focussed significantly longer on chocolate cues $(82.46 \%$ of total trial time) than participants in the attend-shoe condition (6.95\% of total trial time). Thus, the attention modification task yielded significant differences in attention for chocolate between conditions.

Attention modification effects on chocolate consumption, chocolate search time, craving and hunger

At first, independent samples $t$-tests were performed to test whether groups differed with respect to their chocolate intake or their search time. Chocolate intake was first logarithmically transformed because this variable was significantly positively skewed and therefore not normally distributed. Results revealed no effect of condition on chocolate intake, $t(49)=0.05, p=.96$ or on search time $t(49)=0.31, p$ $=.76$, see Table 2. Similarly, independent t-tests on changes in craving and hunger (both variables were entered as difference scores from pre to post attention modification) due to the experimental attention modification condition showed no significant effects of the modification, $t(49)=0.47, p=.64$ for differences in craving and $t(49)=0.03, p=.97$ for differences in hunger, see Table 2 . 
Table 2.

Differences in chocolate consumption, search time for chocolates, and changes in craving and hunger from pre- to post modification, in the "attend shoes" and the "attend chocolate" attention modification conditions, respectively.

\begin{tabular}{|c|c|c|c|c|}
\hline & $\begin{array}{l}\text { Attend Shoes } \\
(n=26) \\
\end{array}$ & $\begin{array}{l}\text { Attend Chocolate } \\
(n=25)\end{array}$ & & \\
\hline & Mean (SD) & Mean (SD) & $t(49)$ & $p$ \\
\hline Chocolate consumption & $8.94(12.23)$ & $9.26(15.41)$ & 0.05 & .96 \\
\hline Search time for chocolates & $3.81(2.25)$ & $3.61(2.49)$ & 0.31 & .76 \\
\hline Changes in Craving & $6.85(14.40)$ & $4.10(26.32)$ & 0.47 & .64 \\
\hline Changes in Hunger & $5.54(14.01)$ & $5.72(24.95)$ & -0.03 & .97 \\
\hline
\end{tabular}

Note. Chocolate consumption is indicated in grams (but statistics are based on log-transformed chocolate consumption), search time is indicated in minutes searched for hidden chocolates, changes in craving indexes the differences from self-reported craving at baseline to self-reported craving after the attention modification task, changes in hunger refer to the difference from self-reported hunger at baseline to self-reported hunger after the attention modification task.

In a next step we tested whether accuracy (i.e., overall percentage of correctly performed trials) moderated the effect of the experimental attention modification task on our outcome measures. We conducted a hierarchical linear regression analysis on logarithmically transformed chocolate intake with the experimental group (as dummy variable) and accuracy (centred) in step 1 and the interaction term of both variables entered in step 2. In Step 1, this regression model showed that condition and accuracy did not predict chocolate consumption. However, in Step 2 of the model there was a significant interaction of accuracy and experimental condition on chocolate intake $(p=.017)$. Exact statistics are displayed in Table 3.

Table 3.

Summary of the hierarchical linear regression model of variables predicting chocolate consumption.

\begin{tabular}{lcccc}
\hline Variable & B & SEB & $\beta$ & $\mathrm{p}$ \\
\hline Step 1 & & & & \\
1. Condition & .010 & .157 & .010 & .948 \\
2. Accuracy (\%) & -.002 & .010 & -.022 & .883 \\
Step 2 & & & \\
1. Condition & .006 & .149 & .005 & .969 \\
2. Accuracy (\%) & -.024 & .014 & -.339 & .079 \\
3. Condition $\times$ Accuracy (\%) & .049 & .020 & .466 & .017 \\
\hline
\end{tabular}

Note. $R^{2}$ for Step $1=.001$ ( $p=$ n.s.); $\Delta R^{2}$ for Step $2=.12(p=.017)$. 
As can be seen by means of simple slope testing, plotted in Figure 2, participants with higher accuracy ( $+1 \mathrm{SD}$ ) tended to eat more chocolate when their attention was modified towards chocolate stimuli and ate less chocolate when-their attention was modified towards shoe stimuli, $\beta=.35, t(47)=1.80, p=.078$ (two-tailed). The reversed pattern was seen for participants with lower accuracy (-1SD): participants who were trained to attend to shoes tended to eat more chocolate than participants whose attention was modified to attend to chocolate, $\beta=-.34, t(47)=$ $1.72, p=.091$ (two-tailed).

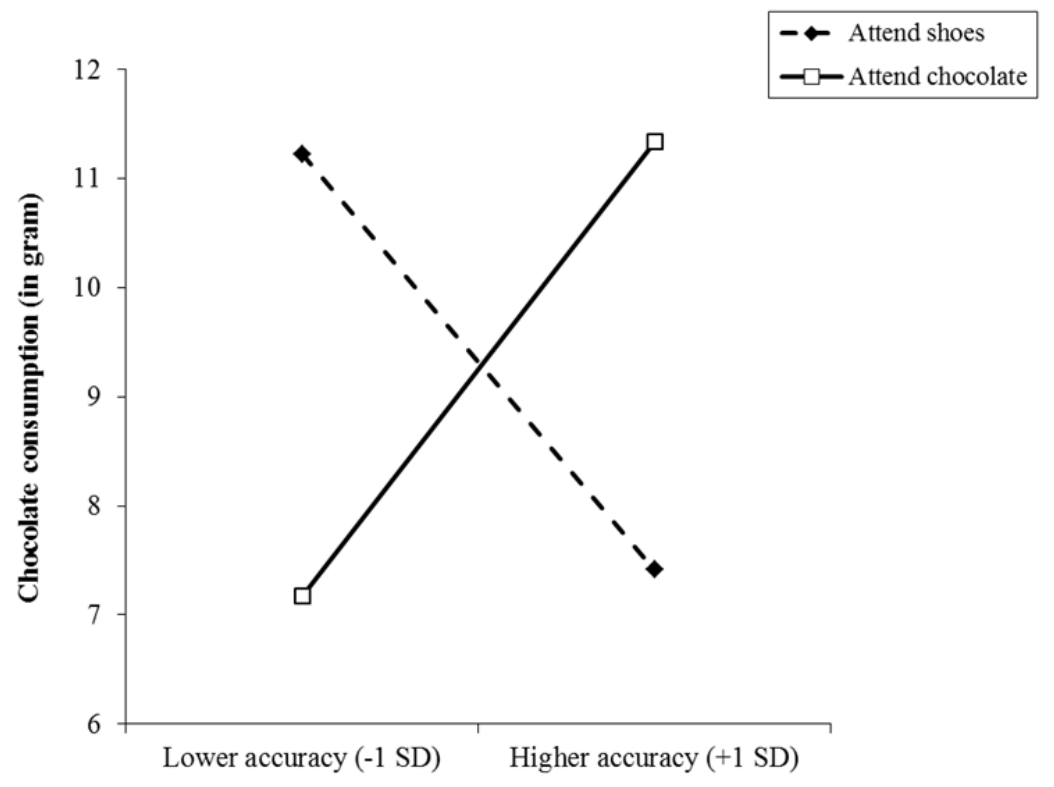

Figure 2. Chocolate consumption (in gram) as a function of higher (+ $1 \mathrm{SD}$ ) and lower (- 1SD) accuracy for the "attend shoe" and the "attend chocolate" condition, respectively.

To further analyse this interaction effect, we probed the effects of accuracy on chocolate intake within each condition ("attend shoe" or "attend chocolate"). Results revealed a trend, in that participants with lower accuracy differed from participants with higher accuracy regarding their chocolate intake in the attend shoe condition, $\beta=-.34, t(47)=1.80, p=.079$ (two-tailed), and in the attend-chocolate condition, $\beta=.35, t(47)=1.71, p=.094$ (two-tailed).

To understand the meaning of higher and lower accuracy on the effect of the attention modification, we first examined the percentage of accuracy (i.e. rate of correctly performed pro - and anti-saccadic trials) in participants with higher and in participants with lower accuracy. Participants with higher accuracy $(+1 S D)$ had a mean of $89.90 \%$ of correctly performed trials and participants with lower accu- 
racy $(-1 S D)$ had a mean of $74.79 \%$ correctly performed trials during the attention modification. To further explore the relation of accuracy and attention modification on chocolate consumption, an additional regression analysis was conducted testing if these differences in accuracy and their impact on chocolate intake could be explained by differences in attention for chocolate. For this aim, a hierarchical regression analysis for dwell time on chocolate as dependent variable was performed with accuracy (centred) and condition (as dummy variable), both entered in Step 1, and the interaction term of these variables, entered in Step 2, as independent predictors. In Step 1, this regression model showed a significant main effect of condition on the time participants dwelled on chocolate images during the attention modification task, as was previously shown in an independent t-test. In addition, in Step 2 of the model there was a significant interaction of accuracy and experimental condition on dwell time on chocolate ( $p=.007)$. Yet, adding the accuracy $\times$ condition interaction to the model was only associated with a minimal increase in total explained variance $\left(\Delta R^{2}\right.$ for Step $2=.003$; Table 4$)$.

Table 4.

Summary of the hierarchical linear regression model of variables predicting dwell time on chocolate (as percentage score of mean total dwell time per trial).

\begin{tabular}{lllll}
\hline Variable & B & SEB & $\beta$ & p \\
\hline Step 1 & & & .99 & .000 \\
1. Condition & 75.87 & 1.73 & -.036 & .119 \\
2. Accuracy (\%) & -0.18 & 0.12 & & .991 \\
Step 2 & & & -000 \\
1. Condition & 75.81 & 1.61 & -.091 & .003 \\
2. Accuracy (\%) & -0.46 & 0.15 & .081 & .007 \\
3. Condition $\times$ Accuracy (\%) & 0.61 & 0.22 & & \\
\hline
\end{tabular}

Note. $\mathrm{R}^{2}$ for Step $1=.976(\mathrm{p}<.001) ; \Delta \mathrm{R}^{2}$ for Step $2=.003(\mathrm{p}=.007)$.

Simple slopes, as displayed in Figure 3, showed that the effect of condition on dwell time for chocolate was overwhelmingly large, for both higher (+ $1 \mathrm{SD})$ and lower accuracy (- 1 SD), ts $>30.80, p s<.001$. The results of this post-hoc analysis suggest that moderating effects of accuracy during the task on chocolate intake are unlikely to be explained by differences in attention for chocolate.

To test whether search time for hidden chocolate differed between conditions, we conducted another hierarchical linear regression analysis with the experimental condition (as dummy variable) and accuracy (centred) in step 1 and the interaction term of both variables entered in step 2. The regression analysis 
showed no significant main effects or interaction predicting search time (all $\beta \mathrm{s}<-$ .07 , all $t \mathrm{~s}<-.47$, all $p \mathrm{~s}>.65$ ), indicating that the attention modification condition and/or accuracy did not affect search times for chocolates.

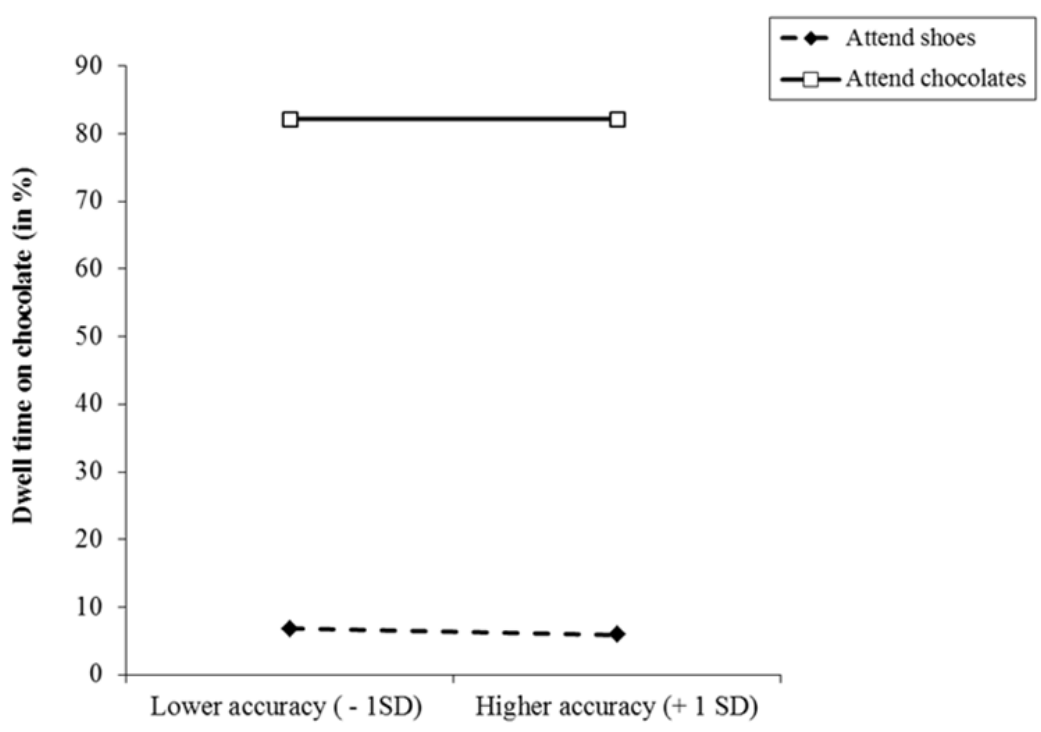

Figure 3. Mean percentage of dwell time on chocolate during chocolate trials as a function of higher (+ $1 \mathrm{SD}$ ) and lower (-1SD) accuracy for the "attend shoe" and the "attend chocolate" condition, respectively.

Third, to test whether accuracy moderated the effect of the experimental attention modification condition on changes in craving or hunger, two hierarchical linear regression analyses for differences in craving or hunger as dependent variables (computed as differences between pre and post indication of craving and hunger on the VAS scales) were performed. In Step 1, the experimental condition (as dummy variable) and accuracy (centred) was entered, and the interaction term of both variables was entered in step 2 . In both analyses, no significant effects of experimental attention modification condition and/or accuracy were found on changes in craving or hunger, all $\beta s<.09$, all $t s<0.52$, all $p s>.61$ for differences in craving; all $\beta s<.27$, all $t s<1.37$, all $p s>.18$ for differences in hunger).

\section{Discussion}

The aim of the present study was to investigate the influence of attention for chocolate on chocolate consumption in the laboratory. In line with our hypothesis, the current results suggest that modifying attention for chocolate cues affects chocolate consumption, yet the accuracy of saccadic eye-movements according to the task instructions had a moderating influence on this effect. Our results showed that 
participants with higher accuracy ate more chocolate when they had to attend to chocolate and ate less chocolate when they had to attend to non-food stimuli. Exactly the reverse was found for participants with lower accuracy. At the moment, we can only speculate on what might have caused differences in accuracy. In this respect it is noteworthy that we did not find a significant association of eating related variables (such as BMI, restrained eating or chronic chocolate craving) and accuracy. This means that differences in accuracy are probably not the result of differences in BMI status, chronic chocolate craving or restrained eating behavior. Yet, it is possible that an underlying attention bias for chocolate or differences in executive or impulse control might have contributed to differences in attention modification accuracy and chocolate consumption. Note that the experimental attention modification and/or accuracy had no effect, however, on craving or search time for chocolates.

Moreover, results regarding chocolate intake should be interpreted cautiously because the present study compared the effects of two manipulations with each other, which makes it difficult to determine whether the observed effect on intake was actually driven by an increase in consumption in the "attend chocolate" condition or by a decrease of consumption in the "attend shoes" condition.

Even though the aim of this study was finding a proof for the hypothesized relation of attention for food and food intake and applied for this aim an attention modification task, this novel paradigm could be interesting in terms of an application in a more clinical study or setting. In general, the ABM task in the present study differed from previous ABM training procedures in two respects: first, the current task modified visual attention directly. In the typically used visual probe task, participants need to look at both stimuli in each trial to evaluate where to direct their attention. In contrast, a particular strength of the current task is that participants are required to voluntarily control their saccadic eye movements and therefore actively avoid or approach one or the other category of task stimuli. In comparison to former $\mathrm{ABM}$ procedures that manipulated attention processes through training of manual response latencies, this novel approach might be more effective because visual attention is trained directly. For example, another current study (Hardman, Rogers, Etchells, Houstoun, \& Munafò) that tried to manipulate attention biases for food by means of the response-latency-based visual probe task failed to produce significant changes in food intake. Second, another advantage of this novel approach to modify attention in the current study was the recording of eye-movements during the task. The recordings of eye movements enabled us to account for the participant's compliance with the task instructions (i.e., accuracy).

Besides, it is also possible that the applied anti-saccade task is more ecologically valid in comparison to the visual probe task with response latency measurements, because the visual attention allocation participants have to perform during this modification task might mirror how participants would decide how to 
(dis)engage attention to food cues in their natural environment: data from a pilot study suggested that participants were able to recognize the stimuli peripherally, even when they had to look away from them. However, future research should test whether the current attention modification procedure was successful in inducing a longer-lasting attentional bias for food cues, for example by testing how participants perform in a subsequent attention paradigm. Moreover, it could also be interesting for future research to determine how attention modification for food cues relates to experimental trainings that are addressing implicit processes with the aim to reduce food intake (e.g. inhibition training, see e.g. Houben \& Jansen (2011))

The findings of the present study contrast somewhat with Smith and Rieger (2009), who found that participants who were manipulated to attend high-calorie food words choose more often low-calorie over high-calorie cookies, after a body image challenge, in comparison to participants who attended neutral words. The aim of the present study diverged from the aim of the study by Smith and Rieger (2009) who focused on (general) food choice. Moreover, the body image challenge as used by Smith and Rieger (2009) might have activated dieting goals, thereby possibly contributing to an increase in calorie-conscious food decisions afterwards (Anschutz, Van Strien, \& Engels, 2011; Papies \& Hamstra, 2010). In contrast, in our study no body image measures were included and we also decided to measure eating behaviour characteristics after the taste test to avoid inducing any dieting or restraint association (Papies \& Hamstra, 2010), which might have counteracted or influenced the pure effects of the ABM training. In this respect, it could be interesting for future studies to examine whether ABM influences both the amount of food consumed and food choice.

Whereas the preliminary evidence of the applied attention modification for chocolate intake seems promising for future attention modification trainings it is notable that the ABM procedure did not affect the amount of time that participants searched for chocolate nor did attention for chocolate affect craving for chocolate or subjective hunger. A likely explanation for this lack of an effect regarding search time could be that this search task might not accurately assess chocolate motivation. One explanation for the lack of an effect on craving could be that participants were asked to indicate their craving for food in general and not specifically for chocolate. Research has shown that food cravings are specifically related to the kind of food consumed (Martin, O'Neil, Tollefson, Greenway, \& White, 2008). Therefore, future studies should test the effects of attention modification on craving with more specific measures. In line with this, studies exploring ABM for appetitive stimuli in addiction research have reported inconsistent findings regarding the effects of ABM on craving (e.g., Attwood, O'Sullivan, Leonards, Mackintosh, \& Munafò, 2008; Field et al., 2007; Field, Duka, Tyler, \& Schoenmakers, 2009; Field \& Eastwood, 2005; Schoenmakers, Wiers, Jones, Bruce, \& Jansen, 2007). Moreover, 
previous research in the field of addiction research indicated that the extent to which participants are aware of the training contingencies can influence their behaviour. For example, Attwood and colleagues (2008) and Field and colleagues (2007) observed ABM effects only in contingency-aware participants. In the present study, we did not assess contingency awareness of participants explicitly but only probed for suspicion. None of the participants indicated that she knew that chocolate consumption was measured, and therefore it seems unlikely that participants ate more or less chocolate because they were aware of the modification contingencies. Yet, future studies should consider measuring contingency awareness. Another important point to note, when interpreting the current findings is that our results apply to a specific food item: chocolate. It would be interesting to determine whether the current findings generalize from ABM for chocolate stimuli to other high-calorie food stimuli or whether ABM for a certain food condition generalizes to other types of food. In this respect, it might be interesting for future studies to investigate the impact using a similar attention modification procedure contrasting high calorie, palatable food with low-calorie food stimuli on subsequent food consumption, as this would be most similar with food exposure in our real food environment.

In conclusion, the results showed that attention bias modification towards and away from chocolate stimuli was related to the amount of chocolate consumption, but only when attention modification was accurately, thereby providing preliminary evidence for an influence of attention for food on subsequent food intake, which might not necessarily be mediated by (conscious) changes in craving. Yet, the issue of causality remains because accuracy in the attention modification task interacted with the effect of modified attention on chocolate versus shoes on subsequent chocolate intake in the current study.

The results of an additional post-hoc analysis suggest that the moderating effect of accuracy on chocolate consumption is not driven by differences in dwelltime on chocolate. That is, dwell-time on chocolate versus shoes was almost completely determined by the modification condition, and hardly depended on the accuracy. This suggests that another (unmeasured) characteristic of the high versus the low accuracy performers determines whether the attention modification either works or does not work for them with regard to their chocolate intake.

Nevertheless, the current finding suggests important theoretical implications for the role of attention on food intake, in that paying attention to food could form a risk for weight gain, particularly when living in an obesogenic food environment. In this respect, knowledge about the working mechanisms of attention and food consumption could have potential clinical benefits. Future research should therefore aim to extend the current findings, thereby further clarifying whether attention modification can impact food intake in general and examining the stability of effects. 


\section{Chapter 8}

General discussion 
CHAPTER 8 
The central idea of this thesis is that attention bias for food affects eating behaviour and weight gain. The studies presented in this thesis were conducted to systematically investigate how attentional components (early and later attentional processes) and the direction of an attention bias (attentional approach versus attentional avoidance) relate to BMI differences in children and adults, appetitive motivation and eating restraint, craving for food, food intake and weight change over time.

First, a brief summary of findings is presented: Results on how an attention bias for food relates to BMI differences are reviewed, then findings about how differences in eating motivation and eating restraint relate to attention biases for food are described, followed by an overview of findings addressing the (causal) relation of attention biases for food, craving, food intake and weight change. Then, methodological considerations concerning the measurement of attention bias and the choice for stimuli sets are briefly mentioned. A conclusion section follows, in which a model for the role of attention bias for food on (over)eating, obesity and weight gain is proposed.

\section{Attention bias for food: in everybody's focus}

Overall, our studies indicate that attention biases for food cues are a rather universal phenomenon. In other words everyone has an attention bias for high-calorie food cues. Our first study showed that all participants (i.e., healthy and overweight female students, after having eaten a small lunch), directed their first gaze significantly more often towards food than neutral stimuli, even though overweight participants directed their first gaze significantly more often towards food than healthy weight participants (see Chapter 2). Similarly, all children, irrespective of their weight status, paid initially (i.e. initial orientation and initial gaze fixation) more attention towards food pictures than towards equally-liked animal pictures, see Chapter 3. Moreover, in samples of mostly healthy weight female students, see Chapter 4, Chapter 5 (additional analyses) and Chapter 6 (additional analyses), everyone paid more attention to food than non-food cues, as indicated by a more frequent initial orientation towards food, longer initial fixation durations on food, longer total dwell time on food and faster response latencies ${ }^{13}$ when the probe replaced a food cue compared to a non-food cue. Apparently, food cues are highly salient for everyone and attract automatically more attention than neutral and even than other attractive non-food cues.

\footnotetext{
13 trend-significant $(p=.08$ ) in the neutral mood condition, additional analyses for data described in Chapter 6
} 
The role of attention bias for food cues in overweight adults and obese children

In addition to our findings that everybody pays more attention to food than other cues, we also found evidence for BMI-related individual differences in attention bias for food, in adults. In one study (Chapter 2), we compared selective attention for food between overweight and healthy weight students when both groups were fed. Our results showed that overweight participants first fixated more often on food images than on non-food images as compared to healthy weight participants. Yet, overweight participants showed diminished attentional maintenance in their initial fixations on food compared to non-food stimuli, in contrast to healthy weight participants. We interpreted these findings in terms of an approachavoidance reaction during a relatively early attention process. According to theory, attention biases for food could be reflecting either eating restraint (that is worry about eating and calorie concern) or appetitive motivation. Our results specify that it could be both: due to the accurate disentanglement of attentional components and the ability to specify the direction of attention allocation (towards versus away from food), our findings suggest that overweight adults in this study had an initial attentional approach of food cues, reflecting appetitive motivation for food, which was quickly followed by the attempt to suppress attentional processing of highcalorie palatable food cues, possibly reflecting eating restraint, thus the wish to avoid food or gain control over food intake.

In contrast to these results in adults, in our study with obese and healthy weight children no differences in attentional processing for food cues were found (Chapter 3). All children initially paid more attention to food than to non-food cues, independent of weight-status and no differences were found in later stages of attention processing. This finding is particularly remarkable because food stimuli were matched in attractiveness to non-food stimuli, which depicted animals. Our interpretation of this finding was that all children might possess an "innate" attention bias for food. However, we speculated that children can unlearn this bias in the course of their development: children who grow up with a healthy weight might learn to attenuate their biased attention for food, whereas children with overweight or obese weight might not learn to regulate their automatic approach tendency, which is then reflected in increased initial orientation for food, as evidenced in obese and overweight adults (e.g. in Chapter 2). This speculation is dovetailed by the finding that automatic attention for food was related to weight gain, at least in obese children (this finding will be discussed later on).

With regard to the role of an food-related attention bias for BMI status, these studies provide two suggestions: first, it is possible that having a tendency to automatically direct attention selectively towards food cues reflects strong appetitive motivation for food, as evidenced by increased initial orientation in overweight participants and that increased initial orientation towards food might be related to 
increasing weight, as suggested by the longitudinal data of obese children. Second, findings also suggest that attention bias for food can change in the course of development: whereas overweight adults initially oriented more often towards highcalorie foods than did healthy-weight adults, all children, independent of their BMI- status, expressed similar food related biases in initial phases of attention.

\section{The role of attention bias in appetitive eating motivation, eating restraint and} emotional eating

Even though our research indicates that food cues are more salient than non-food cues for everyone, we wanted to study how individual differences in eating motivation and eating restraint contribute to specific attention allocation patterns for food, food cravings and food intake. For this aim, we examined attention bias in healthy weight restrained and unrestrained eaters (Chapter 4), manipulated eating allowance and eating forbiddance (Chapter 5) and induced negative mood to study if an attention bias for food is a cognitive mechanism that could contribute to eating under negative emotions (Chapter 6).

In our study on attention bias for food in overweight and healthy weight participants (Chapter 2), we were intrigued by the finding that overweight adults seemed to avoid food cues to a greater extent than healthy weight adults (bias in initial fixation duration). Yet, we also noted that overweight participants scored significantly higher on (chronic) dietary restraint than did healthy weight participants. Theoretically, eating restraint (or dieting) could contribute to increased attention bias for high-calorie food cues, because the dieter or restrained eater might be preoccupied with thoughts about "forbidden" but tempting food. Yet, it is also possible that dieting or eating restraint leads to attentional avoidance in an attempt to escape or to overcome food temptations. Previous research showed evidence for both theories (see for a summary of conflicting findings, Chapter 4).

However, an interpretation of previous findings was complicated by the fact that chronic dietary restraint and weight are often highly correlated. That is, in most previous studies restrained eaters were significantly heavier than unrestrained eaters. Therefore, in an attempt to disentangle effects of chronic dietary restraint from effects of being overweight or obese on attentional processing of food cues, we studied attention bias for food in weight-matched healthy weight restrained and unrestrained eaters (Chapter 4). In our study, all participants, irrespective of their dietary restraint status, showed more selective attention for food than neutral cues, and no differences in attention bias for food emerged between unrestrained and restrained eaters when having a healthy weight. Apparently, restrained eaters with a healthy weight attend to food cues in the same way as unrestrained eaters with a healthy weight. These results extend previous findings 
indicating that overweight restrained females show an approach-avoidance pattern when looking at food (see Chapter 2). Together, the results of both studies suggest that biased attention processing in restrained eaters is to a stronger extent attributable to weight problems than to dietary restraint. Possibly, only dietary restraint in combination with overweight and obesity influences the perception of the food environment in a biased way, and therefore in overweight and obese populations restraint is indeed related to attention biases.

One could further speculate that successful restrained eating (as indicated by having a healthy weight) might contribute to a down-regulation of attention for food, thereby decreasing to a "healthy" level of attention allocation for food. In contrast, overweight individuals with eating restraint, who struggle with their weight and control over food intake, show specific attention bias patterns for food. The observed approach-avoidance mechanism could reflect their initial temptation and preoccupation with tempting food followed by the attempt to escape this temptation by avoiding looking at food.

To further explore the relation of dieting or eating restraint and attention for food we strived to investigate the (causal) impact of allowing oneself to eat versus not permitting oneself to eat. Accordingly, we attempted to manipulate eating (thus appetitive) motivation versus eating restraint to study how these differences in motivation affect attention bias for food cues in mainly healthy weight female students (see Chapter 5). Our findings showed that a brief, verbal manipulation of eating allowance versus eating forbiddance did not affect attentional processing of food cues. However, individual trait-like differences in eating motivation, that is chronic chocolate craving and self-endorsed permission to eat, were related to differences in attention bias for chocolate. Interestingly, opposing attention patterns emerged with regard to these opposing eating motivations: participants who were high chronic chocolate cravers had elevated attentional approach bias for chocolate cues, whereas participants who indicated that they did not allow themselves to eat chocolate showed reduced attention for food. These differences in attentional processing were mainly observed in later stages of attention, participants did not differ in their initial orientation towards food. This finding suggests that conscious decisions about eating are reflected in later attention components and influence the direction of the attention bias: if someone allows oneself to eat, attention is maintained on the food cues, whereas if someone does not permit oneself to eat, attention is not maintained, maybe in an attempt to suppress further processing of tempting food in order to be able to resist the temptation.

We also aimed to investigate how individual differences in mood states impact selective attention for food and food intake. In another study, testing if emotional eating influences attention bias for food and food intake during negative mood, initial orientation for food was significantly affected by experimentally manipulated mood (see Chapter 6). Experimentally induced negative mood led to a decrease 
in initial orientation towards food, a finding that is in line with recent evidence showing that negative mood states are related to decreased reward processing (e.g., Foti \& Hajcak, 2010).Yet, emotional eating, as a trait-like eating-related variable that has previously been associated with uncontrolled eating behaviour during emotional states, was not found to be related to increased attention bias for food (see Chapter 6). Our results further specified that emotional eating was also not related to craving or food intake, neither in an experimental negative nor in a neutral mood. Yet, results showed that in a negative mood attention processing of food cues was reduced in all participants thereby suggesting a general decrease in eating motivation when being sad.

To sum up, our findings regarding the role of differences in eating motivation and eating restraint on attention bias for food stress how important it is to account for individual differences in eating motivation versus eating restraint, weight and mood. Our results regarding dietary restraint resolve a previous controversy on the impact of chronic dietary restraint on attentional processing of food cues: Chronic dietary restraint, in itself without the combination with weight problems, seems to relate to a "unbiased" processing of food cues (or at least not significantly enhanced attentional processing of food cues). Moreover, our results provide evidence that particularly later components of attention, indicating more conscious and wilful control of attentional processing, were associated with individual differences in eating motivation (allowing oneself to eat/high craving food versus not permitting oneself to eat/low craving for food). Moreover, individual differences in mood states seem to impact an early attentional process, as evidenced by the result that a negative mood reduced the ability to detect rewarding (food) cues.

\section{The effect of attention for food on craving and subsequent food intake}

\section{Evidence from correlational data}

Besides investigating how BMI differences and (trait or state) differences in eating motivation affect biased attention for food, we were also interested in examining how selective attention for food relates to craving for food and food intake. In four studies (see Chapters 2, 5-7), attention for food was measured and craving for food and food intake were assessed. Fed overweight participants had elevated initial orientation towards food and this bias was positively associated with craving for food (Chapter 2). In a sample of healthy weight participants (see Chapter 5) initial fixation duration, the dwell time on chocolate pictures and attention bias scores based on response latencies were related to self-reports of momentary chocolate craving. Moreover, all attention biases scores (for chocolate cues) were found to be significantly positively related to subsequent chocolate consumption, in this study. Similarly, another study showed that in a neutral mood, longer atten- 
tion maintenance on food cues (i.e., dwell time) was associated with subsequent higher calorie intake (see Chapter 6).

Thus, in these studies attention bias for food related positively to food cravings and food intake. Results moreover indicate that specifically later attentional processes were most consistently related with food intake and craving, at least in healthy weight female samples when in a neutral mood and tested in a laboratory. Together, findings of these studies represent correlational evidence for an association of attention bias for food with craving and food intake.

\section{Evidence from experimental and longitudinal data}

To gain more knowledge on the impact of attention on food intake we conducted one study with the aim to experimentally modify attention towards food and measured food intake, see Chapter 7. We found that participants ate more chocolate when they had to attend to chocolate and ate less chocolate when they had to attend to non-food stimuli (i.e., shoes). Yet, this finding was limited to participants with high accuracy levels during the attention manipulation. In contrast, the results were exactly reversed for participants with lower accuracy during the attention modification. Curiously, accuracy levels were marginally related to differences in attention for chocolate between attention modification conditions (attend food versus attend shoes ${ }^{14}$. Moreover, no significant effects of the experimental attention modification on craving or search time for hidden chocolates were found. Thus, even though results of this study indicate that directing attention towards food is related to subsequent food intake, the causal issue remains because the cause behind the moderating influence of accuracy during attention modification is not clear. We can only speculate that an underlying, not measured, variable (e.g., impulsivity) contributes to differences in accuracy and then interacts with the attention modification condition on food intake.

As discussed previously, we measured attention bias for food in children and assessed weight change in (a sub-sample of) obese children, to gain more insights on the role of attention bias for food and weight gain (see Chapter 3). Results of this study showed that initial orientation towards food positively predicted weight gain. In contrast to correlational findings, discussed above, it is important to note that weight gain was associated with an early attention component. This contrasts with previous evidence from this thesis indicating that later attentional components, namely maintained attention, were associated with intake-related differences. In sum, our research suggests that there might be two "pathways" through which attention bias for food can influence subsequent food intake and body weight: a bias in later attention processes could be related to conscious decisions

${ }_{14}$ The significant interaction effect of accuracy $\times$ attention modification condition on attention for chocolate showed only a minimal contribution of accuracy and a major contribution of condition, see Chapter 7. 
to indulge, whereas a bias in early attention processes could be related to a less conscious, maybe latent, appetitive motivation. In this respect, particularly increased initial orientation for food might be a vulnerability factor for gradual overconsumption, and thus subsequent weight gain. In contrast, regulating later attentional processes might have more impact in conscious eating situations. This finding stresses the need to distinguish between automatic and voluntary attention processes.

\section{Methodological considerations}

One methodological important aspect of the current thesis concerns the direct versus the indirect assessment of attention bias for food. In all studies eye-tracking was used as direct measure of visual attention and response latencies were assessed as indirect measure of attention (except in Chapter 7). In all our studies, response latency based attention bias did not add information compared to eyetracking based attention biases. Moreover, in some studies attention bias scores based on response latencies seemed not sensitive enough to capture important mechanisms or group differences.

For example, two studies yielded significant differences in attention bias for food due to group characteristics (e.g., overweight versus healthy weight, Chapter 2 or high and low chocolate craving, see Chapter 5). These differences in attention for food were observed on eye-movement based attention biases. In contrast, in the same studies no significant differences in attention for food cues were found between these groups on response latency-based attention biases. Moreover, even though response latency based attention biases were sometimes found to be significantly correlated with food craving and food intake (e.g. Chapter 5), all direct measures of attention (based on eye-tracking) yielded the same information consistently. Therefore, we can safely conclude that eye-tracking as a basis for the calculation of attention biases is more accurate and more sensitive for capturing visual attention processes. This observation is in line with attention bias research in the field of anxiety and addition (e.g., Bannerman, Milders, de Gelder, \& Sahraie, 2009; Field \& Christiansen, 2012; Field, Munafó, \& Franken, 2009; Kirchner \& Thorpe, 2006). Yet, this conclusion is limited by the fact, that we studied response latencies with long presentation duration during the visual probe task, thereby providing an indirect index for maintained attention and therefore this conclusion might not apply for response latencies based on shorter presentation durations (e.g., $100 \mathrm{~ms}$ ).

Another important methodological aspect of this thesis concerns the selection of stimuli for the attention paradigm. Pictures of high-calorie food stimuli were used in all paradigms. Low-calorie food stimuli were not used in order to avoid 
that "dieting" or "health" associations would be evoked (see Chapter 1 for a more extensive explanation). Typically, relevant stimuli (in our case thus food stimuli) are contrasted with pictures of office supplies as neutral contrast category (e.g., Nijs, Muris, Euser, \& Franken, 2010). However, in the current thesis pictures of musical instruments were used in four studies (see Chapter 2, Chapters 4-6). The reason for this choice was that office supplies might not be seen as purely neutral: office supplies are dull and in that sense even negative when contrasted with something positive such as food. In contrast, musical instruments might be viewed as more neutral or even slightly appealing stimuli. This choice enabled us to study if attention for food is increased even if another neutral (but not completely dull) stimulus is available. In two studies (see Chapter 3 and Chapter 7) we chose to use even more attractive objects as neutral stimulus category. In the study described in Chapter 3, we explicitly tested that the non-food contrast category (animals) was also highly liked in a small pilot study. This methodological choice enabled us to study if food is preferentially processed even though attractive stimuli are available. In the study described in Chapter 7 the inclusion of an equally attractive contrast category for chocolate (i.e., shoes) was important to ensure that the effort during the attention modification was comparable in both groups (participants in both conditions were trained to look towards something "nice"). Thus, our research extends earlier research, which contrasted food with relatively "dull" contrast stimuli (office supplies), by testing if food is still preferentially processed when contrasted with relatively neutral non-food stimuli (musical instruments) or with highly attractive non-food stimuli (animals and shoes).

\section{Conclusions}

- Everybody has an attention bias for food, even when food is contrasted with equally attractive stimuli.

- Initial orientation for food is an automatic attention mechanism that is specifically related to a higher BMI in adults and weight gain in obese children after six months.

- Attention maintenance is related to differences in eating motivation (appetitive motivation/eating restraint) and subsequent food intake:

- Differences in the direction of attention (approach versus avoidance) reflect motivational intentions about food intake:

- Attentional approach relates to the (relatively) voluntary decision to allow oneself to eat, specifically in later stages of attentional processing.

- $\quad$ Reduced attention or even attentional avoidance in later stages of attentional processing might reflect the intention not to indulge, and to refrain from eating. 
- Maintaining attention on food is positively related to the amount of food intake.

- Manipulating attention can influence food intake, but only when the manipulation is followed highly consistently:

- Looking at food increases intake.

- Different motivations regarding food consumption are reflected at different stages of information processing:

- Early (involuntary) attention reflects heightened (intrinsic) motivation for food.

- Later (more controlled) attention reflects conscious decisions about food intake (at least in a laboratory situation).

\section{Future research}

The research presented in the current thesis suggests that an attention bias for food facilitates (over)eating and can contribute to weight gain. For future research it would be interesting to investigate how variations in eating restraint in obese participants relate to differences in attentional processing, how successful weight loss is related to differences in voluntary processing of food cues and how differences in attention bias for food relate to weight changes in obese adults and healthy weight children. Together, this information could contribute to a better understanding of the working mechanisms through which an attention bias for food influences eating behaviour and weight gain.

Attention bias for food in overweight and obese participants: take a look at restraint

Our research showed that an attention bias for food is different in overweight when compared to healthy weight individuals (see Chapter 2). The observed pattern in our study suggested an approach-avoidance reaction to food cues in overweight participants. A similar approach-avoidance pattern has been observed in anxiety and addiction research (Garner, Mogg, \& Bradley, 2006; Noël et al., 2006). In social anxiety research this reaction has been interpreted in terms of vigilance for social cues followed by avoidance as strategy to decrease possible discomfort in a social situation and to discourage others (e.g., by avoiding their gaze) from further social interaction. A study on attentional processing of alcohol cues in abstinent alcoholics observed that increased initial attention towards alcohol cues was followed by attentional avoidance in abstinent alcoholics (e.g., Noël, et al., 2006). Abstinent alcoholics might be similar to overweight participants with high restraint intentions (as observed in the sample, see Chapter 2) in that both groups try to refrain from something tempting (alcohol and high-calorie food). According- 
ly, we suggest a similar process: increased pre-occupation and desire for tempting food might be reflected in early orientation (i.e. vigilance) for these food cues, yet further processing of these cues is strategically suppressed as attempt to resist the temptation of food (see Chapter 2). In this respect, our finding provides an explanation for the mixed findings from previous studies that yielded evidence for approach biases in obese participants (e.g., Castellanos et al., 2009) or avoidance of food cues related to overweight and obesity (e.g., Gearhardt, Treat, Hollingworth, \& Corbin, 2012). Our research suggests that the observation of attentional approach or avoidance for food in people with overweight might depend on the strength of current dietary restraint. Thus, besides weight status, another factor should be taken in account, when measuring visual attention for food: eating restraint or dieting might attenuate otherwise increased attention for food. It remains to be tested if attention for food is generally increased in obese individuals (as suggested in the study by Castellanos et al. 2009) and how this increased attention is influenced by eating restraint (as suggested by our own results, see Chapter 2) in obese and overweight individuals over time. To fully understand how an attention bias for food persists with increasing BMI and decreasing eating restraint, future research should test how differences in attention bias for food relate to differences in eating motivation (eating enjoyment versus restrained eating) in overweight and in obese participants, thereby taking the impact of different attention bias components (early versus late) into account. When assessing eating restraint, stimuli selection has to be carefully reviewed to avoid priming participants by showing low-calorie food together with high-calorie food, see the section on our methodological considerations (this Chapter and Chapter 1).

\section{Eating motivation is reflected in voluntary attentional processing}

Another contribution of our research concerns the influence of eating motivation and eating restraint in healthy weight participants on attentional processing of food cues. Our research provided evidence that high intrinsic eating motivation (for example craving, eating allowance, and overeating) is related to attentional approach for food whereas eating restraint (for example: not allowing oneself to eat) is related to reduced attention or even attentional avoidance (see for example Chapter 5, see also Chapter 5 for an extensive discussion of these findings). It is important to note that attentional avoidance was observed in later stages of attentional processing thereby suggesting some kind of conscious or voluntary control over attention allocation towards food (see also LaBerge, 2002). Yet, it has not been tested how adherence to a strict diet when motivated to lose weight influences attentional processing of food cues. To understand how dieting success (losing weight) is related to (voluntary) changes in attention bias for tempting versus 
healthy food, it would be fruitful for future research to examine attention biases over the course of (successful) weight loss. This information could be helpful for designing effective attention bias (re)trainings, in which individuals can learn to focus their attention on healthy food or avoid looking at tempting food.

\section{Attention bias for food contributes to food intake and weight gain}

The research presented in the current thesis extends earlier research by examining the role of an attention bias for food on weight gain in children longitudinally (Chapter 3) and experimentally by manipulating attention for food (Chapter 7). Our finding that an attention bias for food relates to weight gain in children dovetails previous research in mainly healthy weight adults, which showed that Stroop interference for unhealthy food words was related to weight gain after one year, (Calitri, et al., 2010). The indirect measurement of attention bias through the Stroop task makes it difficult to compare findings and do not shed light on the attentional component that underlies weight gain in adults (see Chapter 1 for a brief discussion of the Stroop task). Yet, the two studies complement each other, in that Calitri et al. (2010) tested mainly healthy weight adults, whereas our results are based on obese children. Future research should investigate how weight change is affected by attentional biases for food in healthy and obese adults and children, thereby exploring the role of initial attention and maintained attention.

With regard to the experimental manipulation of attention, it is interesting to note that accuracy moderated our findings and future research should thus further examine what caused differences in accuracy levels and how this contributed to differences in food intake. Altogether, our research on attention bias predicting weight gain (Chapter 3) and our research on attention modification and subsequent food intake (Chapter 7) suggests that having an attention bias towards food influences eating and possibly contributes to subsequent weight gain. Future research should thus further extend our studies by examining the causal relation of attention for food on food intake and subsequent weight gain and identify possible moderators of this relation. 


\title{
In Closing: Don't pay attention to high-calorie foods for a healthy weight!
}

\author{
A model for the role of attention components and the direction of attention on food \\ intake and weight gain
}

Theoretically, it has been suggested that paying attention to desired cues might enhance desire (or craving) and thus increase the chance of intake (Kavanagh, Andrade, \& May, 2005). Our research, presented here in this thesis, supports this notion. Increased attention for food could be a crucial factor for subsequent intake, as also previously suggested by an addiction-like model on the role of attention bias for food and food intake (Nijs \& Franken, 2012). We extend this previous model by suggesting two major specifications based on our findings. First, a distinction between early and later attentional components should be incorporated thereby integrating their distinctive contribution to food intake and weight gain. Second, the direction of attention for food should be considered with regard to differences in eating motivation versus eating restraint, thereby influencing eating behaviour.

With respect to attentional components, we propose, in contrast to the earlier model that did not make this distinction, that early versus later attentional processes mirror differences in more unconscious versus conscious attraction towards food. For example, our research showed that a conscious decision about food intake, allowing oneself to eat versus not allowing oneself to eat, is reflected in later components of attention (see Chapter 5). Later attentional processes indicate a more voluntary stage of information processing and we therefore suggest that these later attention components are more strongly related to craving and immediate food intake.

Initial orientation towards food, in contrast, might reflect increased, yet maybe relatively implicit, attraction towards food, on which is not necessarily acted (at least not in the laboratory), but which contributes over time to weight gain. For example, someone with increased initial attention towards food cues might notice more food cues in the environment. This person might therefore be more often in a situation when she/he has to decide whether to eat or not to eat in comparison to another person that notices food less often. Thus, we think that initial orientation towards food influences food intake more implicitly, less directly in comparison to later, more conscious, stages of attentional processes. We further suggest that this is why initial orientation was found to be related to weight gain (see Chapter 3) and might therefore play an important role in the development of overweight and obesity. Initial orientation towards food could thus be a marker of increased vulnerability for weight gain. Moreover, based on our results, we suggest that the link of initial orientation for food and weight is bi-directional. For example, being 
overweight (or obese) contributes to enhanced initial orientation (as our results showed in Chapter 2) which in turn increases the risk to act on noticing food temptations thereby contributing to generally more food intake and subsequent weight gain (as evidenced by results shown in Chapter 3) - a self-perpetuating circle.

Another adjustment of the model refers to the proposition that the direction of attention matters. We observed attentional approach in individuals who were chronic cravers and attentional avoidance in individuals who reported eating restraint (see e.g. Chapter 2 and Chapter 5). Moreover, there was a positive relation of maintained attention and the amount of food intake. These findings suggest that the direction of attention is important for the amount of subsequent food consumption. Accordingly, we propose that eating restraint (or dieting) reduces attentional approach tendencies towards food and might even lead to attentional avoidance, specifically in later more conscious and controlled processes of attention allocation (see Chapter 5). This attentional avoidance could lead to a decrease in food consumption. In contrast, high eating motivation (such as craving or eating enjoyment) might be reflected in elevated attentional approach for food and could lead to elevation of food consumption (see Chapter 5 and Chapter 7).

Together, our model specifies how voluntary versus more involuntary attention allocation towards (or away) from food influences imminent food intake and long term weight gain.

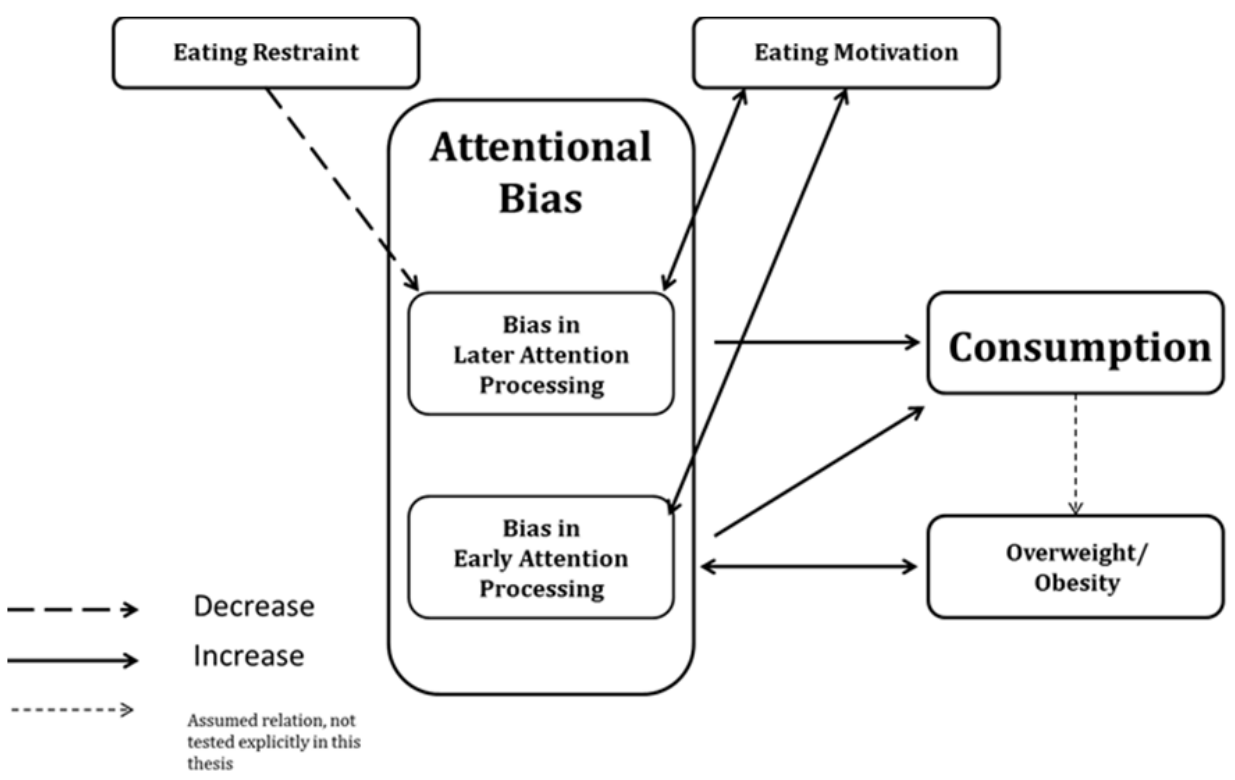

Figure 1. A model for the association of attention for food on craving, food intake and BMI 

Summary 
SUMMARY 
Obesity is a growing epidemic that probably arises to a major future health problem world-wide. Obesity can be seen as a behavioural problem, because it is caused mainly by the individual's inability to regulate food intake and keep a balance of energy expenditure and energy intake. Our environment contributes to the difficulty of healthy eating and healthy weight control by constantly tempting us into indulgence of palatable yet fattening foods.

It has been argued that an attention bias for food could be a cognitive factor that contributes to a vulnerability for overeating. However, individual trait-like eating characteristics might influence how attention is paid to food and how an attention bias for food relates to actual eating behaviour. The central idea of the present dissertation was that paying selectively attention to food is related to eating behaviour, food intake and obesity. One aim of the research conducted for this thesis was to systematically investigate whether an attention bias for food is increased in adults and children with overweight and/or obesity in contrast to individuals with a healthy weight and if eating restraint versus eating enjoyment motivations influence an attention bias for food. Another aim was to test how selective attention for food relates (causally) to actual eating behaviour (in the lab) and to weight gain.

A strength of the current thesis was the consistent application of eyemovement recordings to determine visual attention biases for food. Currently, eyetracking is the most sensible method to measure visual attention processes and calculate attention biases because it allows for a dynamic and accurate disentanglement of early versus later attention components (e.g. initial orientation versus maintained attention) and for the assessment of approach versus avoidance processes.

To address the first aim, my co-authors and I conducted two studies in which attention bias for food was compared between groups differing significantly in their weight. In the first study, we compared attention bias to food in healthy weight and overweight participants (see Chapter 2). Here we found that overweight participants directed their initial attention more often towards high-calorie food but did then not maintain their initial attention on food, in contrast to healthy weight participants. This finding was interpreted as an approach-avoidance reaction towards high-calorie food in overweight participants. Overweight participants might feel initially automatically attracted towards palatable food, but they might also hold strong dieting beliefs (as was qualified by high restrained scores). In the next study on attention bias and weight differences, attention bias for food was tested in obese versus healthy weight children. Children did not differ in their attention bias for food: all children directed their initial attention more towards food cues than towards equally liked animal pictures. However, directing attention towards food predicted further weight gain in obese children after 6 months. Together with the study on attention bias for food in overweight adults, this finding 
suggests that attention for food changes in the course of development (all children hold an attentional bias for food, yet in adults attention bias for food differs based on weight differences). Moreover, this result emphasizes that paying selectively attention for food is associated with weight gain, at least in obese children. Importantly, it was the early attention bias component that predicted weight gain in children.

To further test how differences in eating motivation and eating restraint can impact attention bias for food, my co-authors and I conducted three studies, in which we tested the influence of (chronic) restrained eating within healthy weight participants (Chapter 4), the influence of eating allowance for chocolate (chapter 5 ) and the influence of emotional eating on attentional processing of food cues (Chapter 6). In the study on the impact of restrained eating on attention bias, we wanted to disentangle the relation of restrained eating and BMI and determine the sole impact of restrained eating on attention bias for food (within a healthy weight range). Results showed that restrained eating was not related to biased attention for food within a sample of healthy weight participants (Chapter 4). However, both, restrained and unrestrained healthy weight females showed increased attention bias for food in comparison to neutral stimuli. This finding provides evidence that biased attention for food might occur mainly in relation to weight problems and not in response to restraint intentions, per se. Moreover this result suggests that everybody has to some extent more attention for food than for neutral stimuli in our environment, at least when being healthy weight.

Another study that we conducted showed that a brief instruction intended to manipulate perceived availability of chocolate did not influence attention bias for chocolate cues. Yet, trait-like eating variables did affect attention bias for chocolates: participants who were chronic chocolate cravers showed increased attention bias for food whereas participants who indicated that they would not have allowed themselves to eat chocolate showed diminished attention for food. These attention differences were observed in later attention stages (i.e., maintained attention), associated with a voluntary control over attention. Moreover, attention bias for chocolate was positively related to the amount of subsequent chocolate consumption. This result suggests that trait-like eating variables relate to a voluntary steering of attention towards or away from food cues and that paying selectively attention to food relates to the amount of subsequent food consumption.

In the next study, we tested how emotional eating affects attention bias for food and food intake by inducing a negative mood state experimentally. In this study, maintained attention for food was predictive of the amount of subsequent food intake during a neutral but not in a negative mood. However, emotional eating, a trait-like eating variable that has previously been hypothesized to lead to increased food intake during negative mood, was not predictive of attention bias for food or food intake during negative mood. This result suggests that emotional 
eating might not be as an adequate predictor of food intake during negative mood as previously assumed. Moreover, this finding stresses again the influence of maintained attention on subsequent food consumption.

Finally, to test the causal relation of attention bias for food and subsequent food intake, attention bias was experimentally manipulated towards food or towards shoes and subsequent food intake was measured. Moreover, eyemovements were monitored to account for accuracy during the attention manipulation as possible moderator on effects. This was the first study that monitored visual attention during an attention modification procedure. Results showed that only for participants with high accuracy rates the hypothesized relation was found: Participants who had to attend to chocolate ate more chocolate than participants who had to attend to shoes. However, for participants with lower accuracy rates this relation was exactly reversed. This finding led to the conclusion that attention for food is indeed related to food intake, however the issue of causality remains because we did not identify underlying factors that contribute to differences in accuracy.

Together, these findings stress mainly the role of attentional processing on subsequent food intake and suggest that automatically attending to food cues could be related to future weight gain. Moreover, the research conducted for this thesis highlights the need to distinguish not only between different (temporal) attention bias components but also to take different motivations (eating enjoyment versus dieting intentions) and their impact on attentional processing (approach versus avoidance) into account. In this respect, the conducted studies suggest that differences in eating motivations (eating enjoyment versus eating restraint) are related to voluntary steering of attention towards or away from food. This is important regarding the finding that paying selectively attention to food influences food intake. Future studies should further investigate how voluntary control over attention relates to dieting success and control over food intake.

Based on the research presented in this thesis, it can be concluded that paying selectively attention for food can increase subsequent food intake. However, differences in motivation to eat versus eating restraint impact voluntary control over attention for food. This thesis thus suggests a cognitive mechanism, that is biased attention, by which our toxic food environment can influence our eating behaviour and tempt us into overeating. 

References 
REFERENCES 
Adriaanse, M. A., de Ridder, D. T. D., \& Evers, C. (2011). Emotional eating: Eating when emotional or emotional about eating? [Article]. Psychology \& Health, 26(1), 23-39. doi: 10.1080/08870440903207627

Ahern, A. L., Field, M., Yokum, S., Bohon, C., \& Stice, E. (2010). Relation of dietary restraint scores to cognitive biases and reward sensitivity. Appetite, 55. doi: 10.1016/j.appet.2010.04.001

Allison, D. B., \& Heshka, S. (1993). Social desirability and response bias in self-reports of "emotional eating". Eating Disorders, 1(1), 31-38. doi: 10.1080/10640269308248264

Anderson, B. A., Laurent, P. A., \& Yantis, S. (2013). Reward predictions bias attentional selection. Front Hum Neurosci, 7(262), 00262.

Andreyeva, T., Long, M. W., Henderson, K. E., \& Grode, G. M. (2010). Trying to lose weight: diet strategies among Americans with overweight or obesity in 1996 and 2003. J Am Diet Assoc, 110(4), 535-542.

Anschutz, D. J., Van Strien, T., \& Engels, R. C. M. E. (2011). Exposure to slim images in mass media: Television commercials as reminders of restriction in restrained eaters. Psychology of Popular Media Culture, 1(S), 48-59. doi: 10.1037/2160-4134.1.s.48

Asmaro, D., Jaspers-Fayer, F., Sramko, V., Taake, I., Carolan, P., \& Liotti, M. (2012). Spatiotemporal dynamics of the hedonic processing of chocolate images in individuals with and without trait chocolate craving. Appetite, 58(3), 790-799. doi: 10.1016/j.appet.2012.01.030

Attwood, A. S., O'Sullivan, H., Leonards, U., Mackintosh, B., \& Munafò, M. R. (2008). Attentional bias training and cue reactivity in cigarette smokers. Addiction, 103(11), 1875-1882. doi: 10.1111/j.1360-0443.2008.02335.x

Ayres, K., Prestwich, A., Conner, M., \& Smith, P. (2011). Emotional eating moderates the relationship between implicit measures of attitudes and chocolate consumption. European Journal of Personality, 25(5), 317-325. doi: 10.1002/per.793

Bannerman, R. L., Milders, M., de Gelder, B., \& Sahraie, A. (2009). Orienting to threat: faster localization of fearful facial expressions and body postures revealed by saccadic eye movements. Proc Biol Sci, 276(1662), 1635-1641.

Bannerman, R. L., Milders, M., \& Sahraie, A. (2010). Attentional bias to brief threat-related faces revealed by saccadic eye movements. Emotion, 10(5), 733-738.

Bar-Haim, Y. (2010). Research review: Attention bias modification (ABM): A novel treatment for anxiety disorders. Journal of Child Psychology and Psychiatry, 51(8), 859-870. doi: 10.1111/j.14697610.2010.02251.x

Bar-Haim, Y., Morag, I., \& Glickman, S. (2011). Training anxious children to disengage attention from threat: A randomized controlled trial. Journal of Child Psychology and Psychiatry, 52(8), 861-869. doi: 10.1111/j.1469-7610.2011.02368.x

Benton, D., Greenfield, K., \& Morgan, M. (1998). The development of the attitudes to chocolate questionnaire. Personality and Individual Differences, 24(4), 513-520. doi: 10.1016/s01918869(97)00215-8

Berridge, K. C. (1996). Food reward: Brain substrates of wanting and liking. Neuroscience \& Biobehavioral Reviews, 20,1-25. doi: 10.1016/0149-7634(95)00033-B

Berridge, K. C. (2009). 'Liking' and 'wanting' food rewards: Brain substrates and roles in eating disorders. Physiology \& Behavior, 97(5), 537-550. doi: 10.1016/j.physbeh.2009.02.044

Blechert, J., Feige, B., Hajcak, G., \& Tuschen-Caffier, B. (2010). To eat or not to eat? Availability of food modulates the electrocortical response to food pictures in restrained eaters. Appetite, 54(2), 262268. doi: 10.1016/j.appet.2009.11.007

Bongers, P., Jansen, A., Havermans, R., Roefs, A., \& Nederkoorn, C. (2013). Happy eating: the underestimated role of overeating in a positive mood. Appetite, 67, 74-80.

Bongers, P., Jansen, A., Houben, K., \& Roefs, A. Happy Eating: The Single Target Implicit Association Test predicts overeating after positive emotions. Eating Behaviors(0). doi: http://dx.doi.org/10.1016/j.eatbeh.2013.06.007 
Boon, B., Vogelzang, L., \& Jansen, A. (2000). Do restrained eaters show attention toward or away from food, shape and weight stimuli? European Eating Disorders Review, 8, 51-58. doi: 10.1002/(SICI)1099-0968(200002)8:1<51::AID-ERV306>3.0.CO;2-E

Bradley, B. P., Mogg, K., \& Millar, N. H. (2000). Covert and overt orienting of attention to emotional faces in anxiety. [Article]. Cognition \& Emotion, 14(6), 789-808. doi: 10.1080/02699930050156636

Bradley, B. P., Mogg, K., Wright, T., \& Field, M. (2003). Attentional bias in drug dependence: Vigilance for cigarette-related cues in smokers. Psychology of Addictive Behaviors, 17(1), 66-72. doi: 10.1037/0893-164X.17.1.66

Braet, C., \& Crombez, G. (2003). Cognitive interference due to food cues in childhood obesity. Journal of Clinical Child and Adolescent Psychology, 32(1), 32-39.

Breiner, M. J., Stritzke, W. G. K., \& Lang, A. R. (1999). Approaching avoidance: A step essential to the understanding of craving. Alcohol Research \& Health, 23(3), 197-206.

Brignell, C., Griffiths, T., Bradley, B. P., \& Mogg, K. (2009). Attentional and approach biases for pictorial food cues. Influence of external eating. Appetite, 52(2), 299-306. doi: 10.1016/j.appet.2008.10.007

Brooks, S., Prince, A., Stahl, D., Campbell, I. C., \& Treasure, J. (2011). A systematic review and metaanalysis of cognitive bias to food stimuli in people with disordered eating behaviour. Clinical Psychology Review, 31, 37-51. doi: 10.1016/j.cpr.2010.09.006

Cali, A. M., \& Caprio, S. (2008). Obesity in children and adolescents. J Clin Endocrinol Metab, 93(11 Suppl 1), 2008-1363.

Calitri, R., Pothos, E. M., Tapper, K., Brunstrom, J. M., \& Rogers, P. J. (2010). Cognitive biases to healthy and unhealthy food words predict change in BMI. Obesity, 18(12), 2282-2287.

Canetti, L., Bachar, E., \& Berry, E. M. (2002). Food and emotion. Behavioural Processes, 60(2), 157-164.

Cartwright, F., \& Stritzke, W. G. K. (2008). A multidimensional ambivalence model of chocolate craving: Construct validity and associations with chocolate consumption and disordered eating. Eating Behaviors, 9(1), 1-12.

Carver, C. S., \& White, T. L. (1994). Behavioral Inhibition, Behavioral Activation, and Affective Responses to Impending Reward and Punishment: The BIS/BAS Scales. [Article]. Journal of Personality \& Social Psychology, 67(2), 319-333.

Castellanos, E. H., Charboneau, E., Dietrich, M. S., Park, S., Bradley, B. P., Mogg, K., \& Cowan, R. L. (2009). Obese adults have visual attention bias for food cue images: evidence for altered reward system function. [Article]. International Journal of Obesity, 33(9), 1063-1073. doi: 10.1038/ijo.2009.138

Centraal Bureau voor de Statistiek. (2013). Report over "Leefstijl, preventief onderzoek; persoonskenmerken." (2013). Leefstijl, preventief onderzoek; persoonskenmerken. Retrieved from http://statline.cbs.nl/StatWeb/publication/?VW=T\&DM=SLNL\&PA=81177NED\&D1=14,26,3943\&D2=0-12,33-38\&D3=0\&D4=l\&HD=130129-1607\&HDR=G3,G2,T\&STB=G1.

Conner, M., Fitter, M., \& Fletcher, W. (1999). Stress and snacking: A dary study of dialy hassles and between-meal snacking [Article]. Psychology \& Health, 14(1), 51.

Cox, W. M., Fadardi, J. S., \& Pothos, E. M. (2006). The addiction-stroop test: Theoretical considerations and procedural recommendations. Psychol Bull, 132(3), 443-476.

Davis, C., \& Carter, J. C. (2009). Compulsive overeating as an addiction disorder. A review of theory and evidence. Appetite, 53(1), 1-8. doi: 10.1016/j.appet.2009.05.018

Davis, C., Patte, K., Levitan, R., Reid, C., Tweed, S., \& Curtis, C. (2007). From motivation to behaviour: A model of reward sensitivity, overeating, and food preferences in the risk profile for obesity. Appetite, 48(1), 12-19.

deCastro, J. M., Bellisle, F., Feunekes, G. I. J., Dalix, A. M., \& DeGraaf, C. (1997). Culture and meal patterns: A comparison of the food intake of free-living American, Dutch, and French students. [Article]. Nutrition Research, 17(5), 807-829.

Dobson, K. S., \& Dozois, D. J. A. (2004). Attentional biases in eating disorders: A meta-analytic review of Stroop performance. Clinical Psychology Review, 23, 1001-1022. doi: 10.1016/j.cpr.2003.09.004 
Evers, C., de Ridder, D. T. D., \& Adriaanse, M. A. (2009). Assessing yourself as an emotional eater: mission impossible? Health Psychology: Official Journal of The Division Of Health Psychology, American Psychological Association, 28(6), 717-725. doi: 10.1037/a0016700

Evers, C., Marijn Stok, F., \& de Ridder, D. T. (2010). Feeding your feelings: emotion regulation strategies and emotional eating. Pers Soc Psychol Bull, 36(6), 792-804.

Eyelink 1000 [Apparatus and software]. (2010). Mississauga, Canada: SR Research Ltd.

EyeLink Data Viewer User's Manual (Document Version 1.11.1). (2011). Mississauga, ON, Cananda: SR Research.

Fairburn, C. G., Cooper, Z., Shafran, R., Bohn, K., Hawker, D. M., Murphy, R., . . Fairburn, C. G. (2008). Dietary restraint, dietary rules and controlling eating Cognitive behavior therapy and eating disorders. (pp. 124-135). New York, NY US: Guilford Press.

Faunce, G. J. (2002). Eating disorders and attentional bias: A review. Eating Disorders: The Journal of Treatment \& Prevention, 10, 125-139.

Felix, C. (2010). Dancing with the Sun. In C. d. Mar (Ed.), Volume 16.

Field, M., \& Christiansen, P. (2012). Commentary on, 'Internal reliability of measures of substancerelated cognitive bias'. Drug Alcohol Depend, 124(3), 189-190.

Field, M., \& Cox, W. M. (2008). Attentional bias in addictive behaviors: A review of its development, causes, and consequences. [Review]. Drug and Alcohol Dependence, 97(1-2), 1-20. doi: 10.1016/ j.drugalcdep.2008.03.030

Field, M., Duka, T., Eastwood, B., Child, R., Santarcangelo, M., \& Gayton, M. (2007). Experimental manipulation of attentional biases in heavy drinkers: Do the effects generalise? Psychopharmacology, 192(4), 593-608. doi: 10.1007/s00213-007-0760-9

Field, M., Duka, T., Tyler, E., \& Schoenmakers, T. (2009). Attentional bias modification in tobacco smokers. Nicotine \& Tobacco Research, 11(7), 812-822. doi: 10.1093/ntr/ntp067

Field, M., \& Eastwood, B. (2005). Experimental manipulation of attentional bias increases the motivation to drink alcohol. Psychopharmacology, 183(3), 350-357. doi: 10.1007/s00213-0050202-5

Field, M., Hogarth, L., Bleasdale, D., Wright, P., Fernie, G., \& Christiansen, P. (2011). Alcohol expectancy moderates attentional bias for alcohol cues in light drinkers. Addiction, 106(6), 1097-1103. doi: 10.1111/j.1360-0443.2011.03412.x

Field, M., Mogg, K., \& Bradley, B. P. (2006). Attention to drug-related cues in drug abuse and addiction: component processes. In R. W. Wiers \& A. Stacy, W. (Eds.), Handbook of implicit cognition and addiction. Thousand Oaks: Sage Publications.

Field, M., Mogg, K., Zetteler, J., \& Bradley, B. P. (2004). Attentional biases for alcohol cues in heavy and light social drinkers: the roles of initial orienting and maintained attention. Psychopharmacology, 176(1), 88-93. doi: 10.1007/s00213-004-1855-1

Field, M., Munafó, M. R., \& Franken, I. H. A. (2009). A meta-analytic investigation of the relationship between attentional bias and subjective craving in substance abuse. Psychological Bulletin, 135, 589-607. doi: 10.1037/a0015843

Flint, A., Raben, A., Blundell, J. E., \& Astrup, A. (2000). Reproducibility, power and validity of visual analogue scares in assessment of appetite sensations in single test meal studies. [Article]. International Journal of Obesity, 24(1), 38-48.

Forestell, C. A., Lau, P., Gyurovski, I. I., Dickter, C. L., \& Haque, S. S. (2012). Attentional biases to foods: The effects of caloric content and cognitive restraint. Appetite, 59(3), 748-754. doi: 10.1016/ j.appet.2012.07.006

Foti, D., \& Hajcak, G. (2010). State sadness reduces neural sensitivity to nonrewards versus rewards. NeuroReport: For Rapid Communication of Neuroscience Research, 21(2), 143-147. doi: 10.1097/ WNR.0b013e3283356448

Franken, I. H. A. (2003). Drug craving and addiction: integrating psychological and neuropsychopharmacological approaches. Progress in Neuro-Psychopharmacology \& Biological Psychiatry, 27(4), 563-579. doi: 10.1016/S0278-5846(03)00081-2. 
Franken, I. H. A. (2007). Craving, cue-reactivity, attentional bias and relapse in addiction. European Neuropsychopharmacology, 17, S214-S214.

Frankort, A., Roefs, A., Siep, N., Roebroeck, A., Havermans, R., \& Jansen, A. (2012). Reward activity in satiated overweight women is decreased during unbiased viewing but increased when imagining taste: an event-related fMRI study. International Journal of Obesity (2005), 36(5), 627-637. doi: 10.1038/ijo.2011.213

Frewen, P. A., Dozois, D. J. A., Joanisse, M. F., \& Neufeld, R. W. J. (2008). Selective attention to threat versus reward: Meta-analysis and neural-network modeling of the dot-probe task. Clinical Psychology Review, 28(2), 307-337. doi: 10.1016/j.cpr.2007.05.006

Gearhardt, A. N., Treat, T. A., Hollingworth, A., \& Corbin, W. R. (2012). The relationship between eatingrelated individual differences and visual attention to foods high in added fat and sugar. Eat Behav, 13(4), 371-374.

Giel, K. E., Friederich, H. C., Teufel, M., Hautzinger, M., Enck, P., \& Zipfel, S. (2011). Attentional processing of food pictures in individuals with anorexia nervosa--an eye-tracking study. Biol Psychiatry, $69(7), 661-667$.

Giesen, J. C. A. H., Havermans, R. C., Douven, A., Tekelenburg, M., \& Jansen, A. (2010). Will work for snack food: The association of BMI and snack reinforcement. Obesity, 18, 966-970. doi: 10.1038/oby.2010.20

Graham, R., Hoover, A., Ceballos, N. A., \& Komogortsev, O. (2011). Body mass index moderates gaze orienting biases and pupil diameter to high and low calorie food images. Appetite, 56, 577-586. doi: 10.1016/j.appet.2011.01.029

Hakamata, Y., Lissek, S., Bar-Haim, Y., Britton, J. C., Fox, N. A., Leibenluft, E., . . Pine, D. S. (2010). Attention Bias Modification Treatment: A meta-analysis toward the establishment of novel treatment for anxiety. Biological Psychiatry, 68(11), 982-990. doi: 10.1016/j.biopsych. 2010.07.021

Halford, J. C., Gillespie, J., Brown, V., Pontin, E. E., \& Dovey, T. M. (2004). Effect of television advertisements for foods on food consumption in children. Appetite, 42(2), 221-225.

Hallett, P. E. (1978). Primary and secondary saccades to goals defined by instructions. Vision Research, 18(10), 1279-1296. doi: 10.1016/0042-6989(78)90218-3

Hallett, P. E., \& Adams, B. D. (1980). The predictability of saccadic latency in a novel voluntary oculomotor task. Vision Research, 20(4), 329-339. doi: 10.1016/0042-6989(80)90019-x

Hallion, L. S., \& Ruscio, A. M. (2011). A meta-analysis of the effect of cognitive bias modification on anxiety and depression. Psychological Bulletin, 137(6), 940-958. doi: 10.1037/a0024355

Hardman, C. A., Rogers, P. J., Etchells, K. A., Houstoun, K. V. E., \& Munafò, M. R. The effects of food-related attentional bias training on appetite and food intake. Appetite, 71(0), 295-300. doi: http://dx.doi.org/10.1016/j.appet.2013.08.021

Harriger, J. A., \& Thompson, J. K. (2012). Psychological consequences of obesity: Weight bias and body image in overweight and obese youth. International Review of Psychiatry, 24(3), 247-253. doi: 10.3109/09540261.2012.678817

Harris, J., L., Bargh, J., A., \& Brownell, K., D. (2009). Priming effects of television food adverstising on eating behavior. Health Psychology, 28(4), 404-413. doi: 10.1037/a0014399

Hepworth, R., Mogg, K., Brignell, C., \& Bradley, B. P. (2010). Negative mood increases selective attention to food cues and subjective appetite. Appetite, 54(1), 134-142. doi: 10.1016/j.appet.2009.09.019

Herman, C. P., \& Polivy, J. (1980). Restrained Eating. In A. J. Stunkard (Ed.), Obesity (pp. 209-224). Philadelphia: Saunders.

Hervas, G., \& Vazquez, C. (2013). Low spirits keep rewards subdued: Decreases in sensitivity to reward and vulnerability to dysphoria. Behavior Therapy, 44(1), 62-74. doi: 10.1016/j.beth.2012.07.003

Hetherington, M. M., \& Macdiarmid, J. I. (1993). 'Chocolate addiction': A preliminary study of its description and its relationship to problem eating. Appetite, 21(3), 233-246. doi: 10.1006/appe.1993.1042 
Hickey, C., \& Theeuwes, J. (2011). Context and competition in the capture of visual attention. Atten Percept Psychophys, 73(7), 2053-2064.

Higgs, S. (2007). Impairment of cognitive performance in dietary restrained women when imagining eating is not affected by anticipated consumption. Eating Behaviors, 8(2), 157-161.

Higgs, S., Rutters, F., Thomas, J. M., Naish, K., \& Humphreys, G. W. (2012). Top down modulation of attention to food cues via working memory. Appetite, 59(1), 71-75. doi:10.1016/j.appet. 2012.03.018

Hill, J. O., Catenacci, V., \& Wyatt, H. R. (2005). Obesity: Overview of an Epidemic. Psychiatric Clinics of North America, 28(1), 1-23. doi: 10.1016/j.psc.2004.09.010

Hill, J. O., \& Peters, J. C. (1998). Environmental contributions to the obesity epidemic. Science, 280(5368), 1371-1374. doi: 10.1126/science.280.5368.1371

Hoek, J., \& Gendall, P. (2006). Advertising and Obesity: A Behavioral Perspective. Journal of Health Communication, 11(4), 409-423.

Hofmann, W., \& Van Dillen, L. (2012). Desire: The new hot spot in self-control research. Current Directions in Psychological Science, 21(5), 317-322. doi: 10.1177/0963721412453587

Hollitt, S., Kemps, E., Tiggemann, M., Smeets, E., \& Mills, J. S. (2010). Components of attentional bias for food cues among restrained eaters. Appetite, 54(2), 309-313. doi: 10.1016/j.appet.2009.12.005

Houben, K., \& Jansen, A. (2011). Training inhibitory control. A recipe for resisting sweet temptations. Appetite, 56(2), 345-349.

Houben, K., Roefs, A., \& Jansen, A. (2010). Guilty pleasures: Implicit preferences for high calorie food in restrained eating. Appetite, 55(1). doi: 10.1016/j.appet.2010.03.003

Hu, F. B. (2003). Overweight and Obesity in Women: Health Risks and Consequences. Journal of Women's Health, 12(2), 163-172. doi: 10.1089/154099903321576565

Hutton, S. B. (2008). Cognitive control of saccadic eye movements. Brain and Cognition, 68(3), 327-340. doi: 10.1016/j.bandc.2008.08.021

Jansen, A., Vanreyten, A., van Balveren, T., Roefs, A., Nederkoorn, C., \& Havermans, R. (2008). Negative affect and cue-induced overeating in non-eating disordered obesity. Appetite, 51(3), 556-562.

Jeffery, R. W., Folsom, A. R., Luepker, R. V., Jacobs Jr, D. R., Gillum, R. F., Taylor, H. L., \& Blackburn, H. (1984). Prevalence of Overweight and Weight Loss Behavior in a Metropolitan Adult Population: The Minnesota Heart Survey Experience. [Article]. American Journal of Public Health, 74(4), 349352.

Johnson, F., Pratt, M., \& Wardle, J. (2011). Dietary restraint and self-regulation in eating behavior. International Journal of Obesity, 36, 665-674. doi: 10.1038/ijo.2011.156

Jones, A., Hogarth, L., Christiansen, P., Rose, A. K., Martinovic, J., \& Field, M. (2012). Reward expectancy promotes generalized increases in attentional bias for rewarding stimuli. Quarterly Journal of Experimental Psychology, 65(12), 2333-2342. doi: 10.1080/17470218.2012.686513

Jones, N., \& Rogers, P. J. (2003). Preoccupation, food, and failure: An investigation of cognitive performance deficits in dieters. International Journal of Eating Disorders, 33(2), 185-192. doi: 10.1002/eat.10124

Jordan, A. B., Kramer-Golinkoff, E. K., \& Strasburger, V. C. (2008). Does adolescent media use cause obesity and eating disorders? Adolesc Med State Art Rev, 19(3), 431-449.

Kalarchian, M. A., \& Marcus, M. D. (2012). Psychiatric comorbidity of childhood obesity. International Review of Psychiatry, 24(3), 241-246. doi: 10.3109/09540261.2012.678818

Kavanagh, D. J., Andrade, J., \& May, J. (2005). Imaginary relish and exquisite torture: the elaborated intrusion theory of desire. Psychological Review, 112(2), 446-467.

Keatley, D., Clarke, D. D., \& Hagger, M. S. (2012). Investigating the predictive validity of implicit and explicit measures of motivation in problem-solving behavioural tasks. Br J Soc Psychol, 28(10), 2044-8309.

Kelly, B., Halford, J. C., Boyland, E. J., Chapman, K., Bautista-Castano, I., Berg, C., . . Summerbell, C. (2010). Television food advertising to children: a global perspective. Am J Public Health, 100(9), 1730-1736. 
Kemps, E., \& Tiggemann, M. (2009). Attentional bias for craving-related (chocolate) food cues. Experimental and Clinical Psychopharmacology, 17(6), 425-433. doi: 10.1037/a0017796

Kindt, M., \& Van Den Hout, M. (2001). Selective attention and anxiety: A perspective on developmental issues and the causal status. Journal of Psychopathology and Behavioral Assessment, 23(3), 193202. doi: $10.1023 / \mathrm{a}: 1010921405496$

Kindt, M., van den Hout, M., de Jong, P., \& Hoekzema, B. (2000). Cognitive bias for pictorial and linguistic threat cues in children. Journal of Psychopathology and Behavioral Assessment, 22(2), 201-219. doi: 10.1023/a:1007540608596

Kissler, J., \& Keil, A. (2008). Look-don't look! How emotional pictures affect pro- and anti-saccades. Experimental Brain Research, 188(2), 215-222.

Kirchner, H., \& Thorpe, S. J. (2006). Ultra-rapid object detection with saccadic eye movements: visual processing speed revisited. Vision Res, 46(11), 1762-1776.

Knudsen, E. I. (2007). Fundamental components of attention. Annu Rev Neurosci, 30, 57-78.

LaBerge, D. (2002). Attentional control: brief and prolonged. Psychol Res, 66(4), 220-233.

Lee, M., \& Shafran, R. (2004). Information processing biases in eating disorders. Clinical Psychology Review, 24(2), 215-238. doi: 10.1016/j.cpr.2003.10.004

Loeber, S., Grosshans, M., Korucuoglu, O., Vollmert, C., Vollstadt-Klein, S., Schneider, S., . . Kiefer, F. (2012). Impairment of inhibitory control in response to food-associated cues and attentional bias of obese participants and normal-weight controls. Int J Obes, 36(10), 1334-1339.

Lowe, M. R., Butryn, M. L., Didie, E. R., Annunziato, R. A., Thomas, J. G., Crerand, C. E., . . Halford, J. (2009). The Power of Food Scale. A new measure of the psychological influence of the food environment. Appetite, 53(1), 114-118. doi: 10.1016/j.appet.2009.05.016

Lowe, M. R., \& Levine, A. S. (2005). Eating Motives and the Controversy over Dieting: Eating Less Than Needed versus Less Than Wanted. Obesity Research, 13(5), 797-806.

Loxton, N. J., Dawe, S., \& Cahill, A. (2011). Does negative mood drive the urge to eat? The contribution of negative mood, exposure to food cues and eating style. Appetite, 56(2), 368-374. doi: 10.1016/j.appet.2011.01.011

Macht, M. (2008). How emotions affect eating: A five-way model. Appetite, 50(1), 1-11. doi: 10.1016/ j.appet.2007.07.002

Macht, M., Gerer, J., \& Ellgring, H. (2003). Emotions in overweight and normal-weight women immediately after eating foods differing in energy. Physiology \& Behavior, 80(2-3), 367-374.

MacLeod, C., Mathews, A., \& Tata, P. (1986). Attentional bias in emotional disorders. Journal of Abnormal Psychology, 95(1), 15-20. doi: 10.1037/0021-843x.95.1.15

Martin, C. K., O'Neil, P. M., Tollefson, G., Greenway, F. L., \& White, M. A. (2008). The association between food cravings and consumption of specific foods in a laboratory taste test. Appetite, 51(2), 324326.

Martin, M. (1990). On the induction of mood. Clinical Psychology Review, 10, 669-697.

Mathews, A., \& MacLeod, C. (2002). Induced processing biases have causal effects on anxiety. Cognition and Emotion, 16(3), 331-354.

Meule, A., Vögele, C., \& Kübler, A. (2012). Restrained eating is related to accelerated reaction to high caloric foods and cardiac autonomic dysregulation. Appetite, 58, 638-644. doi: 10.1016/ j.appet.2011.11.023

Miller, A., \& Hom, H. L. (1990). Influence of extrinsic and ego incentive value on persistence after failure and continuing motivation. Journal of Educational Psychology, 82(3), 539-545. doi: 10.1037/ 0022-0663.82.3.539

Mogg, K., Bradley, B. P., De Bono, J., \& Painter, M. (1997). Time course of attentional bias for threat information in non-clinical anxiety. Behaviour Research and Therapy, 35(4), 297-303. doi: http://dx.doi.org/10.1016/S0005-7967(96)00109-X

Mogg, K., Field, M., \& Bradley, B. P. (2005). Attentional and approach biases for smoking cues in smokers: An investigation of competing theoretical views of addiction. Psychopharmacology, 180(2), 333-341. 
Mogg, K., Bradley, B. P., Field, M., \& De Houwer, J. (2003). Eye movements to smoking-related pictures in smokers: Relationship between attentional biases and implicit and explicit measures of stimulus valence. Addiction, 98(6), 825-836.

Mogg, K., Bradley, B. P., Hyare, H., \& Lee, S. (1998). Selective attention to food-related stimuli in hunger: Are attentional biases specific to emotional and psychopathological states, or are they also found in normal drive states? Behaviour Research and Therapy, 36(2), 227-237. doi: 10.1016/S00057967(97)00062-4

Mogg, K., Bradley, B. P., Miles, F., \& Dixon, R. (2004). Time course of attentional bias for threat scenes: Testing the vigilance-avoidance hypothesis. [Article]. Cognition \& Emotion, 18(5), 689-700. doi: 10.1080/02699930341000158

Mokdad, A. H., Marks, J. S., Stroup, D. F., \& Gerberding, J. L. (2004). Actual Causes of Death in the United States, 2000. JAMA: Journal of the American Medical Association, 291(10), 1238-1245.

Munoz, D. P., \& Everling, S. (2004). Look away: the anti-saccade task and the voluntary control of eye movement. Nat Rev Neurosci, 5(3), 218-228. doi: 10.1038/nrn1345

Murdaugh, D. L., Cox, J. E., Cook, E. W., III, \& Weller, R. E. (2012). fMRI reactivity to high-calorie food pictures predicts short- and long-term outcome in a weight-loss program. NeuroImage, 59(3), 2709-2721. doi: 10.1016/j.neuroimage.2011.10.071

Nathan, P. J., O’Neill, B. V., Mogg, K., Bradley, B. P., Beaver, J., Bani, M., . . Bullmore, E. T. (2012). The effects of the dopamine $\mathrm{D}_{3}$ receptor antagonist GSK598809 on attentional bias to palatable food cues in overweight and obese subjects. International Journal of Neuropsychopharmacology, 15(2), 149-161. doi: 10.1017/s1461145711001052

Nederkoorn, C., Coelho, J. S., Guerrieri, R., Houben, K., \& Jansen, A. (2012). Specificity of the failure to inhibit responses in overweight children. Appetite, 59(2), 409-413. doi: 10.1016/j.appet.2012. 05.028

Nijs, I. M. T., \& Franken, I. H. A. (2012). Attentional Processing of Food Cues in Overweight and Obese Individuals. Curr Obes Rep, 1(2), 106-113.

Nijs, I. M. T., Franken, I. H. A., \& Muris, P. (2008). Food cue-elicited brain potentials in obese and healthyweight individuals. Eat Behav, 9(4), 462-470.

Nijs, I. M. T., Franken, I. H. A., \& Muris, P. (2009). Enhanced processing of food-related pictures in female external eaters. Appetite, 53(3), 376-383. doi: 10.1016/j.appet.2009.07.022

Nijs, I. M. T., Franken, I. H. A., \& Muris, P. (2010). Food-related Stroop interference in obese and normalweight individuals: Behavioral and electrophysiological indices. Eating Behaviors, 11(4), 258-265. doi: 10.1016/j.eatbeh.2010.07.002

Nijs, I. M. T., Muris, P., Euser, A. S., \& Franken, I. H. A. (2010). Differences in attention to food and food intake between overweight/obese and normal-weight females under conditions of hunger and satiety. Appetite, 54(2), 243-254. doi: 10.1016/j.appet.2009.11.004

Noël, X., Colmant, M., Van Der Linden, M., Bechara, A., Bullens, Q., Hanak, C., \& Verbanck, P. (2006). Time course of attention for alcohol cues in abstinent alcoholic patients: The role of initial orienting. Alcoholism: Clinical and Experimental Research, 30(11), 1871-1877. doi: 10.1111/j.15300277.2006.00224.x

Nummenmaa, L., Hietanen, J. K., Calvo, M. G., \& Hyona, J. (2011). Food catches the eye but not for everyone: a BMI-contingent attentional bias in rapid detection of nutriments. PLoS One, 6(5), 16.

Overduin, J., Jansen, A., \& Louwerse, E. (1995). Stroop interference and food intake. International Journal of Eating Disorders, 18, 277-285. doi: 10.1002/1098-108X(199511)18:3<277::AIDEAT2260180310>3.0.CO;2-3

Papies, E. K., \& Hamstra, P. (2010). Goal priming and eating behavior: Enhancing self-regulation by environmental cues. Health Psychology, 29(4), 384-388. doi: 10.1037/a0019877

Papies, E. K., Stroebe, W., \& Aarts, H. (2008). The allure of forbidden food: On the role of attention in self-regulation. Journal of Experimental Social Psychology, 44(5), 1283-1292. doi: 10.1016/j.jesp. 2008.04.008 
Phaf, R. H., \& Kan, K. J. (2007). The automaticity of emotional Stroop: a meta-analysis. J Behav Ther Exp Psychiatry, 38(2), 184-199.

Phelan, S., Hassenstab, J., McCaffery, J. M., Sweet, L., Raynor, H. A., Cohen, R. A., \& Wing, R. R. (2011). Cognitive interference from food cues in weight loss maintainers, normal weight, and obese individuals. Obesity, 19(1), 69-73.

Piech, R. M., Pastorino, M. T., \& Zald, D. H. (2010). All I saw was the cake. Hunger effects on attentional capture by visual food cues. Appetite, 54(3), 579-582.

Polivy, J., Herman, C. P., \& Coelho, J. S. (2008). Caloric restriction in the presence of attractive food cues: External cues, eating, and weight. Physiology \& Behavior, 94(5), 729-733.

Pothos, E. M., Tapper, K., \& Calitri, R. (2009). Cognitive and behavioral correlates of BMI among male and female undergraduate students. Appetite, 52(3), 797-800.

Puhl, R. M., \& Heuer, C. A. (2009). The stigma of obesity: A review and update. Obesity, 17(5), 941-964.

Pulgaron, E. R. (2013). Childhood obesity: a review of increased risk for physical and psychological comorbidities. Clin Ther, 35(1), 014.

Rinck, M., Becker, E. S., Kellermann, J., \& Roth, W. T. (2003). Selective attention in anxiety: distraction and enhancement in visual search. Depress Anxiety, 18(1), 18-28.

Robbins, S. J., \& Ehrman, R. N. (2004). The role of attentional bias in substance abuse. Behavioral and Cognitive Neuroscience Reviews, 3(4), 243-260. doi: 10.1177/1534582305275423

Rodin, J., Mancuso, J., Granger, J., \& Nelbach, E. (1991). Food cravings in relation to body mass index, restraint and estradiol levels: A repeated measures study in healthy women. Appetite, 17(3), 177185. doi: 10.1016/0195-6663(91)90020-s

Rodríguez, S., Fernández, M. C., Cepeda-Benito, A., \& Vila, J. (2005). Subjective and physiological reactivity to chocolate images in high and low chocolate cravers. Biological Psychology, 70(1), 9-18.

Roefs, A., Huijding, J., Smulders, F. T., MacLeod, C. M., de Jong, P. J., Wiers, R. W., \& Jansen, A. T. (2011). Implicit measures of association in psychopathology research. Psychol Bull, 137(1), 149-193.

Roefs, A., Quaedackers, L., Werrij, M. Q., Wolters, G., Havermans, R., Nederkoorn, C., ... Jansen, A. (2006). The environment influences whether high-fat foods are associated with palatable or with unhealthy. Behaviour Research and Therapy, 44, 715-736.

Ryan, F. (2002). Detected, selected, and sometimes neglected: Cognitive processing of cues in addiction. [Review]. Experimental and Clinical Psychopharmacology, 10(2), 67-76. doi: 10.1037//10641297.10.2.67

Schoenmakers, T., Wiers, R. W., Jones, B. T., Bruce, G., \& Jansen, A. T. M. (2007). Attentional re-training decreases attentional bias in heavy drinkers without generalization. Addiction, 102(3), 399-405. doi: 10.1111/j.1360-0443.2006.01718.x

Schrauwen, P., \& Westerterp, K. R. (2000). The role of high-fat diets and physical activity in the regulation of body weight. British Journal of Nutrition, 84, 417-427.

Shafran, R., Lee, M., Cooper, Z., Palmer, R. L., \& Fairburn, C. G. (2007). Attentional bias in eating disorders. International Journal of Eating Disorders, 40(4), 369-380. doi: 10.1002/eat.20375

Shafran, R., Lee, M., Cooper, Z., Palmer, R. L., \& Fairburn, C. G. (2008). Effect of psychological treatment on attentional bias in eating disorders. International Journal of Eating Disorders, 41(4), 348-354. doi: 10.1002/eat.20500

Siep, N., Roefs, A., Roebroeck, A., Havermans, R., Bonte, M. L., \& Jansen, A. (2009). Hunger is the best spice: An fMRI study of the effects of attention, hunger and calorie content on food reward processing in the amygdala and orbitofrontal cortex. Behavioural Brain Research, 198(1), 149-158.

Simmons, W. K., Martin, A., \& Barsalou, L. W. (2005). Pictures of Appetizing Foods Activate Gustatory Cortices for Taste and Reward. Cerebral Cortex, 15(10), 1602-1608.

Smeets, E., Roefs, A., \& Jansen, A. (2009). Experimentally induced chocolate craving leads to an attentional bias in increased distraction but not in speeded detection. Appetite, 53(3), 370-375.

Smeets, E., Roefs, A., van Furth, E., \& Jansen, A. (2008). Attentional bias for body and food in eating disorders: increased distraction, speeded detection, or both? Behaviour Research and Therapy, 46(2), 229-238. doi: 10.1016/j.brat.2007.12.003 
Smith, E., \& Rieger, E. (2009). The effect of attentional training on body dissatisfaction and dietary restriction. European Eating Disorders Review, 17(3), 169-176. doi: 10.1002/erv.921

Snoek, H. M., van Strien, T., Janssens, J. M. A. M., \& Engels, R. C. M. E. (2008). Restrained eating and BMI: A longitudinal study among adolescents. Health Psychology, 27(6), 753-759. doi: 10.1037/02786133.27.6.753

Soetens, B., \& Braet, C. (2007). Information processing of food cues in overweight and normal weight adolescents. British Journal Of Health Psychology, 12(Pt 2), 285-304.

Stice, E., Spoor, S., Ng, J., \& Zald, D. H. (2009). Relation of obesity to consummatory and anticipatory food reward. Physiology \& Behavior, 97(5), 551-560.

Stice, E., Yokum, S., Bohon, C., Marti, N., \& Smolen, A. (2010). Reward circuitry responsivity to food predicts future increases in body mass: Moderating effects of DRD2 and DRD4. NeuroImage, 50(4), 1618-1625. doi: 10.1016/j.neuroimage.2010.01.081

Strasburger, V. C. (2011). Children, adolescents, obesity, and the media. Pediatrics, 128(1), 201-208.

Tapper, K., Pothos, E. M., Fadardi, J. S., \& Ziori, E. (2008). Restraint, disinhibition and food-related processing bias. Appetite, 51(2), 335-338. doi: 10.1016/j.appet.2008.03.006

Tetley, A., Brunstrom, J., \& Griffiths, P. (2009). Individual differences in food-cue reactivity. The role of BMI and everyday portion-size selections. Appetite, 52, 614-620.

Thomas, S. L., Hyde, J., Karunaratne, A., Herbert, D., \& Komesaroff, P. A. (2008). Being 'fat' in today's world: A qualitative study of the lived experiences of people with obesity in Australia. Health Expectations: An International Journal of Public Participation in Health Care \& Health Policy, 11(4), 321-330.

Tiffany, S. T. (1999). Cognitive concepts of craving. [Article]. Alcohol Research \& Health, 23(3), 215-224.

Tiggemann, M., \& Kemps, E. (2005). The phenomenology of food cravings: The role of mental imagery. Appetite, 45(3), 305-313.

Timmerman, G. M., \& Gregg, E. K. (2003). Dieting, Perceived Deprivation, and Preoccupation With Food. Western Journal of Nursing Research, 25(4), 405-418. doi: 10.1177/0193945903025004006

Townshend, J. M., \& Duka, T. (2007). Avoidance of alcohol-related stimuli in alcohol-dependent inpatients. Alcoholism: Clinical and Experimental Research, 31(8), 1349-1357. doi: 10.1111/ j.1530-0277.2007.00429.x

Unsworth, N., Schrock, J. C., \& Engle, R. W. (2004). Working memory capacity and the antisaccade task: individual differences in voluntary saccade control. J Exp Psychol Learn Mem Cogn, 30(6), 1302-1321.

van den Hurk, K., van Dommelen, P., van Buuren, S., Verkerk, P. H., \& HiraSing, R. A. (2007). Prevalence of overweight and obesity in the Netherlands in 2003 compared to 1980 and 1997. Archives of Disease in Childhood, 92(11), 992-995. doi: 10.1136/adc.2006.115402

van Strien, T. (2005). Lijnen helpt niet bij emotionele eters. Gedrag \& Gezondheid: Tijdschrift voor Psychologie en Gezondheid, 33(4), 268-276.

Van Strien, T., Cebolla, A., Etchemendy, E., Gutiérrez-Maldonado, J., Ferrer-García, M., Botella, C., \& Baños, R. (2013). Emotional eating and food intake after sadness and joy. Appetite.

van Strien, T., Frijters, J. E. R., Bergers, G. P. A., \& Defares, P. B. (1986). The Dutch Eating Behavior Questionnaire (DEBQ) for Assessment of Restrained Emotional, and External Eating Behavior. International Journal of Eating Disorders, 5(2), 295-315. doi: 10.1002/1098108X(198602)5:2<295::AID-EAT2260050209>3.0.C0;2-T

Van Winckel, M., \& Van Mil, E. (2001). Wanneer is dik te dik? In C. Braet \& M. Van Winckel (Eds.). Behandelingsstrategieen Bij Kinderen Met Overgewicht (pp. 11-26). Houten: Bohn Stafleu Van Loghum.

Van Zant, R. S. (1992). Influence of diet and exercise on energy expenditure-a review. Int J Sport Nutr, 2(1), 1-19.

Veenstra, E. M., de Jong, P. J., Koster, E. H. W., \& Roefs, A. Attentional avoidance of high-fat food in unsuccessful dieters. Journal of Behavior Therapy and Experimental Psychiatry, 41(3), 282-288. 
Vogt, J., De Houwer, J., \& Crombez, G. (2011). Multiple goal management starts with attention: Goal prioritizing affects the allocation of spatial attention to goal-relevant events. Experimental Psychology, 58, 55-61. doi: 10.1027/1618-3169/a000066

Vollstädt-Klein, S., Loeber, S., von der Goltz, C., Mann, K., \& Kiefer, F. (2009). Avoidance of alcoholrelated stimuli increases during the early stage of abstinence in alcohol-dependent patients. Alcohol and Alcoholism, 44(5), 458-463. doi: 10.1093/alcalc/agp056

Wadden, T. A., Brownell, K. D., \& Foster, G. D. (2002). Obesity: responding to the global epidemic. J Consult Clin Psychol, 70(3), 510-525.

Wallis, D. J., \& Hetherington, M. M. (2004). Stress and eating: the effects of ego-threat and cognitive demand on food intake in restrained and emotional eaters. Appetite, 43(1), 39-46.

Wallis, D. J., \& Hetherington, M. M. (2009). Emotions and eating. Self-reported and experimentally induced changes in food intake under stress. Appetite, 52(2), 355-362. doi: 10.1016/j.appet.2008.11.007

Wang, Y., \& Lim, H. (2012). The global childhood obesity epidemic and the association between socioeconomic status and childhood obesity. Int Rev Psychiatry, 24(3), 176-188.

Wardle, J. (1987). Compulsive eating and dietary restraint. British Journal of Clinical Psychology, 26(1), 47-55. doi: 10.1111/j.2044-8260.1987.tb00722.x

Watson, D., Clark, L. A., \& Tellegen, A. (1988). Development and validation of brief measures of positive and negative affect: The PANAS scales. Journal of Personality and Social Psychology, 54(6), 10631070. doi: 10.1037/0022-3514.54.6.1063

Werthmann, J., Roefs, A., Nederkoorn, C., \& Jansen, A. (2013). Desire lies in the eyes: Attention bias for chocolate is related to craving and self-endorsed eating permission. Appetite, 1(13), 0031300319.

Werthmann, J., Roefs, A., Nederkoorn, C., Mogg, K., Bradley, B. P., \& Jansen, A. (2011). Can(not) take my eyes off it: Attention bias for food in overweight participants. Health Psychology, 30, 561-569. doi: $10.1037 / \mathrm{a} 0024291$

Werthmann, J., Roefs, A., Nederkoorn, C., Mogg, K., Bradley, B. P., \& Jansen, A. (2013). Attention bias for food is independent of restraint in healthy weight individuals-An eye tracking study. Eating Behaviors, 14(3), 397-400. doi: 10.1016/j.eatbeh.2013.06.005

Wieser, M. J., Pauli, P., \& Muhlberger, A. (2009). Probing the attentional control theory in social anxiety: an emotional saccade task. Cogn Affect Behav Neurosci, 9(3), 314-322.

Wilcox, R. R. (2011). Fundamentals of modern statistical methods: Substantially improving power and accuracy. New York: Springer.

Williams, J. M., Mathews, A., \& MacLeod, C. (1996). The emotional Stroop task and psychopathology. Psychol Bull, 120(1), 3-24.

Williamson, D. F., Serdula, M. K., Anda, R. F., Levy, A., \& Byers, T. (1992). Weight Loss Attempts in Adults: Goals, Duration, and Rate of Weight Loss. American Journal of Public Health, 82(9), 1251-1251.

Wolters, L. H., de Haan, E., Vervoort, L., Hogendoorn, S. M., Boer, F., \& Prins, P. J. M. (2012). The timecourse of threat processing in children: A temporal dissociation between selective attention and behavioral interference. Anxiety, Stress \& Coping: An International Journal, 25(3), 259-273. doi: 10.1080/10615806.2011.581278

World Health Organization. (1998). Obesity: Preventing and Managing the Global Epidemic. Geneva: World Health Organization.

World Health Organization. (2013a). Obesity and overweight. Fact Sheet 311. Retrieved 02.07.2013, 2013 from http://amro.who.int/common/Display.asp?Lang=E\&RecID=10203

World Health Organization. (2013b). World Health Statistics 2013.

Yeomans, M. R., \& Coughlan, E. (2009). Mood-induced eating. Interactive effects of restraint and tendency to overeat. Appetite, 52(2), 290-298. doi: 10.1016/j.appet.2008.10.006

Yokum, S., Ng, J., \& Stice, E. (2011). Attentional bias to food images associated with elevated weight and future weight gain: An fMRI study. Obesity, 19(9), 1775-1783. doi: 10.1038/oby.2011.168 
Acknowledgements 
ACKNOWLEDGEMENTS 
I would like to thank and acknowledge everyone who helped and supported me and who contributed to my research. You all made my $\mathrm{PhD}$ years an exciting, valuable and great experience.

Anne, Anita and Sjaan: you three are my "godmothers" of research, my PhDproject-trinity, and I am very grateful that I had the opportunity to work together with all three of you on my PhD project, because each of you has her own approach and taught me different lessons about research.

Anne, thank you so much for all your encouragements, for your support, for your optimism concerning publications and for your belief in my work as a researcher. Your work and your way of scientific thinking were a great and very valuable guidance for me. You are the best supervisor I could wish for. Thank you for trusting in my autonomy- and for always being there when I needed your help, your advice and your opinion. I am very happy and grateful that we will continue to work together.

Anita, you are a brilliant promotor and I have learned so much from you, especially about research attitude. Thank you for your great supervision, your funny comments, your critical thoughts and your witty mind when planning research, discussing results or during the writing process. I appreciate all your efforts (e.g. nightly readings) and your great extrinsic (champagne bottles) publicationrewards/incentives. It is a great privilege to work together with you.

Sjaan, thank you so much for being such an enthusiastic "surrogate" supervisor and for all your super-smart ideas and suggestions in the planning and conducting phase of my research, for your feedback and for helping out when I needed more participants by asking your daughters and her friends.

My co-authors in the U.K., Prof. Karin Mogg and Prof. Brendan Bradley from the University of Southampton: I really appreciated your thoughts in the planning phase and your valuable contribution and feedback during the writing process. I have learned a lot from your way of looking at attentional bias. Prof. Matt Field and everyone from his lab in Liverpool: thank you so much for making my stay in Liverpool such a great experience. I learned a lot from our research discussions and I enjoyed our pub-meetings (which were not mutually exclusive). I hope to see you all back a lot in the future. Dear Matt, you are a super quick and super impressive research-thinker and I am happy that we met. You are a great inspiration to me.

Prof. Dr. Lowe from Drexel University in the USA, dear Michael, thank you so much for giving me the opportunity to conduct my research master internship with you and your wonderful group in Philadelphia. You were a great supervisor and prepared me very well for my future job as a PhD student.

Dr. Smits, due to your encouragement I conducted an empirical study for my bachelor thesis at Aarhus University. This research project was a great opportunity 
and made me realize that I wanted to pursue a career in research. Thank you so much for believing in me and trusting me with this research study.

I also want to thank everyone from the eating group for their valuable comments, their "mee-denken" and their ideas on the research results and the research ideas that I presented in the group, and for the great atmosphere! The A-team rocks! Thank you, Carolien, Remco, Katrijn, Janneke, Astrid, Nicolette, Jessica, Peggy, Ghislaine, Ramona, Elke, Hugo, Nele, Karolien, Eva, Harris and Bastian.

Jessie, Angela, Truus, Lindy, Annie and Marionne: Thank you for all your organisational help. Jessie: thank you for never feeling bothered by my emails and requests! The Maastricht eye-tracker-guys from the third floor: Erik, Ron and Johan: thank you so much for helping me with technical eye-tracker problems.

I would like to acknowledge Nora, Lana, Charlotte, Irene, Carmel, Ariane and Ghislaine for helping recruiting and testing participants.

I want to thank the members of my reading committee (Prof. dr. Arnoud Arntz, Prof. dr. Ingmar Franken, Prof. dr. Peter de Jong, Prof. dr. Nanne de Vries, Prof. dr. Madelon Peters) for taking the time to read and to evaluate my dissertation manuscript.

From 2011 to 2013 I had the great opportunity to gather clinical experience while working as a behavioural therapist at the RIAGG Maastricht. This was a very important and valuable experience for me and I would like to thank everyone at the RIAGG for their support. Mijn bijzondere dank geldt natuurlijk voor iedereen van Team 2 (angststoornissen) en mijn begeleiders op de RIAGG. Marisol, een dikke dank voor het introduceren aan de therapeutische werkwijze. Ik heb veel van je geleerd, bijvoorbeeld dat "gewoon praten" heel leuk is maar echt gedragsmatig aan de slag gaan veel leuker (en vooral met therapie succes beloond)! Rene, je was een geweldige supervisor en je zult altijd een groot voorbeeld als therapeut voor mij zijn. Heel veel dank voor je begrip, geduld en voor het aanleren van therapeutische creativiteit (bijvoorbeeld voor het uitdagen). Door jouw begeleiding was het makkelijk om moeilijke stappen in de therapie met vertrouwen te nemen. Kalsoum, dank je wel voor alle ervaringen en vaardigheden die ik door jou heb geleerd, bijvoorbeeld dat je therapiemotivatie niet kan opdringen (ook ben je zelf als therapeut super gemotiveerd $(\dot{)})$.

Janneke and Alana, dear paranimfen: thank you so much for all your advice, your calmness and your motivation during these years. Alana, vielen Dank für deine Geduld und dein Verständnis, vor allem, wenn irgendetwas nicht so läuft wie es sollte. Janneke, je bent sinds ik begonnen ben met mijn PhD-project mijn kamergenoot geweest. Bij jou kon ik altijd terecht met kleine en grotere vragen over onderzoek, werken aan de UM, onderwijsgeven etc. Heel veel dank voor al je adviezen en voor je vriendschap! 
I also want to thank all other PhD students from CPS for making me feel at home at CPS and for all the fun we had on conferences, dinners, during EPP symposia and previous defence parties.

Thank you, roomies (from the third floor: Janneke, Katrijn, Peggy, Karolien), for keeping up with me when I was stressed out or when I had my five minutes of babbling. Lotte, polka-dot-dress-twin, thank you for being a good friend and for staying calm in Marrakech. Astrid, ich vermiss dich hier in Maastricht und hoffe, dass irgendwann eine Zeit kommt in der wir uns räumlich wieder annähern. Kristina, ich fand unsere EPP Gespräche immer total toll und es ist/war wunderbar mit Dir die PhD-Erfahrungen teilen zu können. Anna, mit dir zusammen Kaffee-trinken gehen, hätte ruhig öfters sein sollen (wären da nicht unsere übervollen Agendas gewesen)!

Last, but not least, I want to thank my family and my friends from outside the UM for their open ears and minds and their patience with me during these workloaded last four years.

Allen voran gilt der Dank natürlich meiner Mama, Annette, für ihre großartige Fürsorge, mein Leben lang. Meiner Mama, Dietrich und meiner Oma möchte ich danken für ihre liebe und großzügige Unterstützung während meines Psychologie Studiums. Mama und Dietrich, ich konnte mich immer auf euch verlassen (z.B. wenn ihr mich mitten in der Nacht aus der Uni abgeholt habt, weil unser Auto in der Garage eingeschlossen war) - das war grossartig.

Ich möchte mich auch beim kompletten Rest der Familie bedanken für euer Interesse an meiner Arbeit, dafür dass ihr mir immer zugehört habt und für mich da wart, wenn ich euch brauchte (und nicht den langen Weg nach Aachen gescheut habt, wenn es etwas zu feiern gab). Ich möchte mich auch gerne bei Fritz Familie bedanken: ihr seid eine total tolle Familie und ich bin sehr glücklich, dass ich mich dazu zählen kann. Elisabeth, mit dir über Psychologie (und alle sonstigen Themen der Welt) zu diskutieren und sich aus zu tauschen ist einfach klasse!

Liebe Freunde, vielen Dank, dass ihr mir Rückhalt gegeben habt, mir zugehört habt, mich abgelenkt habt und mich geerdet habt (Liebe Isabell, vielen Dank dafür, dass du immer da bist, wenn ich dich brauche, zum Beispiel, wenn das Auto nachts in der Uni eingeschlossen und der letzte Bus nach Aachen schon gefahren ist)!

Fritz, my beloved husband, without you these years would have been only half as fun, exciting and wonderful as they were with you. Thank you for everything. 



\section{Curriculum Vitae}


CURRICULUM VITAE 
Jessica Werthmann was born in Bochum (Germany) on November 3rd, 1981. In July 2001 she graduated from secondary school at the Couven Gymnasium in Aachen (Germany). Subsequently she studied Political Sciences for three years and obtained her Vor-Diplom at the University of Potsdam, in Germany. She then started a Bachelor education in Mental Health Science at the Faculty of Health, Medicine and Life Science, Maastricht University (The Netherlands). For her Bachelor thesis she spent three months at Aarhus University (Denmark) working on a research project. After obtaining her Bachelor degree, she joined the Research Master in Psychopathology at the Faculty of Psychology and Neuroscience at Maastricht University (The Netherlands) which she completed with cum laude in 2009. During her studies, she followed a clinical internship at the Christoph Dornier Stiftung in Cologne (Germany). She conducted the research for her Master thesis at Drexel University (Philadelphia, Pennsylvania, USA). In September 2009, she started her PhD project at the department of Clinical Psychological Science, Maastricht University. She conducted parts of her research at the University of Liverpool (U.K.) in collaboration with Prof. Dr. Matt Field. During her PhD project she worked halftime as therapist for anxiety disorders at the local academic outpatient clinic (RIAGG) between 2010 and 2013. Since December 2013, she works as a postdoctoral researcher at the Department of Clinical Psychological Science, Maastricht University.

\section{Publications}

Journal articles

Werthmann, J., Field, M., Roefs, A., Nederkoorn, C., \& Jansen, A. (2014). Attention bias for chocolate increases chocolate consumption - An attention bias modification study. Journal of Behavior Therapy and Experimental Psychiatry, 45, 136-143

Werthmann, J., Renner F., Roefs, A. Huibers, M. \& Jansen, A. (in press). Looking at food in sad mood: Do attentional biases lead emotional eaters into overeating after negative mood induction? Eating Behaviors.

Werthmann, J., Roefs, A., Nederkoorn, C., \& Jansen, A. (2013). Desire lies in the eyes. Attention bias for chocolate is related to craving and self-endorsed eating permission. Appetit, 70, 81 - 89.

Werthmann, J., Roefs, A., Nederkoorn, C., Mogg, K., Bradley, B.P. \& Jansen, A. (2011). Can(not) take my eyes off it: Attention bias for food in overweight participants. Health Psychology 30(5), 561-569. 
Werthmann, J., Roefs, A., Nederkoorn, C., Mogg, K., Bradley, B.P. \& Jansen, A. (2013). Attention bias for food is independent of restraint in healthy weight individuals -An eye tracking study. Eating Behaviors, 14, 397-400.

Werthmann, J., Smits, L.J., \& Li, J. (2010). Parental mortality rates in a western country after the death of a child: assessment of the role of the child's sex. Gender Medicine, 7, 39-46.

\section{Submitted manuscripts}

Werthmann, J., Roefs, A. Havermans, R. , Nederkoorn, C., Kremers, S., \& Jansen, A. (2014). Food texture is the hair in the soup for picky eaters - a novel behavioural test for picky eating in young children. Manuscript submitted for publication.

Werthmann, J. Roefs, A., Nederkoorn, C., Schyns, G., \& Jansen, A. (2013). The power of food: attention bias for high-calorie food predicts weight gain in obese children. Manuscript in revision.

\section{Conference presentations}

Werthmann, J., Roefs, A., Nederkoorn, C., Mogg, K., Bradley, B.P. \& Jansen, A. (2010). Can(not) take my eyes off it: Attention bias for food in overweight participants. Poster presented at the British Feeding and Drinking Group (BFDG) Annual Meeting, Maastricht, The Netherlands.

Werthmann, J., Roefs, A., Nederkoorn, C., Mogg, K., Bradley, B.P. \& Jansen, A. (2010). Can(not) take my eyes off it: Attention bias for food in overweight participants. Open Paper presented at the World Congress of Cognitive Behavioral Therapies (WCBCT), Boston, USA.

Werthmann, J., Roefs, A., Nederkoorn, C., Mogg, K., Bradley, B.P. \& Jansen, A. (2010). Aandachtsbias, drang om eten en overeten: raak niet afgeleid door vet eten voor een gezond gewicht. In Havermans, R. (Chair), Eetgenot en - grieven. Symposium presentation at the Dutch Association of Behavior and Cognitive Therapy, Veldhoven, The Netherlands.

Werthmann, J., Roefs, A., Nederkoorn, C., Mogg, K., Bradley, B.P. \& Jansen, A. (2011). Can(not) take my eyes off it: Attention bias for food in overweight participants. Open paper presentation at the Congress of the European Association of Behavioral and Cognitive Therapies (EABCT), Reykjavik, Island.

Werthmann, J., Roefs, A., Nederkoorn, C., Mogg, K., Bradley, B.P. \& Jansen, A. (2011). Essen im Blickpunkt: Aufmerksamkeit, Heißhunger und übermässiges Essen. Symposium presentation at annual congres of the „Deutsche Gesellschaft für Adipositas" (DAG), Bochum, Germany. 
Werthmann, J., Roefs, A., Nederkoorn, C., Mogg, K., Bradley, B.P. \& Jansen, A. (2012). Das Auge isst mit!? Studien zum Zusammenhang zwischen selektiver Aufmerksamkeit, Heißhunger und übermäßigem Essen. In Horndasch S. \& Giel, K. (Chair), Die Verarbeitung störungsspezifischer Stimuli bei Patientinnen mit einer Essstörung. Symposium presentation at annual congres of the „Deutsche Gesellschaft für Essstörungen“ (DGESS), Hannover, Germany.

Werthmann, J., Roefs, A., Nederkoorn, C., Mogg, K., Bradley, B.P. \& Jansen, A. (2012). Can(not) take my eyes off it: Attention bias for food. In Prof. Matt Field (Chair), Beverage, attention and appetitive behaviors. Symposium presentation at the British Feeding and Drinking Group (BFDG) Annual Meeting, Brighton, Great Britain.

Werthmann, J., Roefs, A., Nederkoorn, C., Mogg, K., Bradley, B.P. \& Jansen, A. (2013). Eating is in the eye of the beholder. In Herbert, B. M., \& Blechert, J. (Chair), Mechanisms of eating behavior, food craving and its regulation. Symposium presentation at the Conference of Experimental Psychologists (TeaP), Vienna, Austria.

Werthmann, J., Roefs, A., Nederkoorn, C., Mogg, K., Bradley, B.P. \& Jansen, A. (2013). Attention for fat food - associations with overweight and obesity in adults and children. In De Ruiter, M. (Chair), Watch out, or your gaze takes control! The role of attentional bias in the development, maintenance and treatment of psychological disorders. Symposium presentation at the Annual Congress of the European Association for Behavioral Cognitive Therapy (EABCT), Marrakesh, Morocco. 


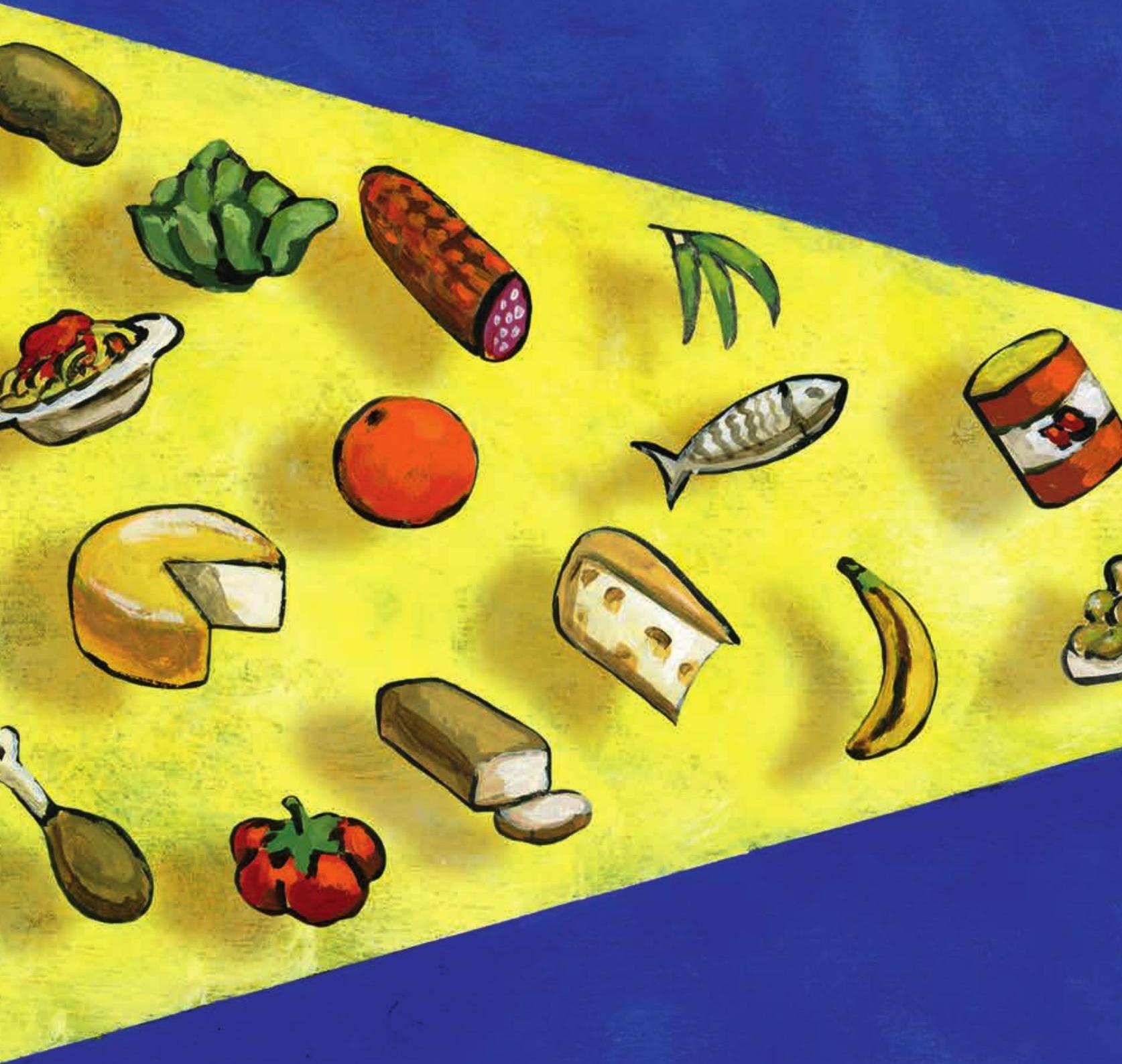

Don't pay attention to high-calorie foods for a healihy weight: attention bias for food and the desire to eat Jessica Werthmann 